\title{
Anti-inflammatory withanolides from physalis minima
}

\author{
Jiangping Wu, ${ }^{\dagger}$ Tao Zhang, ${ }^{\dagger}$ Meng $\mathrm{Yu},{ }^{\dagger}$ Hongmei Jia,,${ }^{\dagger}$ Hongwu Zhang, ${ }^{\dagger}$ \\ Qiongming Xu, ${ }^{\star}$ Yucheng Gu, $*, \S$ and Zhongmei Zou $*, \dagger$
}

†Institute of Medicinal Plant Development, Chinese Academy of Medical Sciences and Peking Union Medical College, Beijing 100193, China;

$\$$ College of Pharmaceutical Science, Soochow University, Suzhou 215123, China;

§Syngenta, Jealott's Hill International Research Centre, Berkshire RE42 6EY, UK.

* Corresponding author: Zhongmei Zou, Yucheng Gu, PhD;

Tel/Fax: 86-1057833290

E-mail address: zmzou@implad.ac.cn; yucheng.gu@syngenta.com 
S4 Table S1 ${ }^{13} \mathrm{C}-\mathrm{NMR}(150 \mathrm{MHz})$ spectroscopic data for compounds $\mathbf{6}-\mathbf{1 0}$ in $\mathrm{CD}_{3} \mathrm{OD}$.

Table S2 ${ }^{1} \mathrm{H}-\mathrm{NMR}(600 \mathrm{MHz})$ spectroscopic data for compounds 6-10 in $\mathrm{CD}_{3} \mathrm{OD}$.

HR-ESI-MS spectrum of compound 1, ${ }^{1} \mathrm{H}$ NMR spectrum of compound $\mathbf{1}$ in $\mathrm{CD}_{3} \mathrm{OD}$ at $600 \mathrm{MHz}$.

Detailed ${ }^{1} \mathrm{H}$ NMR spectrum of compound 1 in $\mathrm{CD}_{3} \mathrm{OD}$ at $600 \mathrm{MHz}$, Detailed ${ }^{1} \mathrm{H}$ NMR spectrum of compound 1 in $\mathrm{CD}_{3} \mathrm{OD}$ at $600 \mathrm{MHz}$.

${ }^{13} \mathrm{C}$-NMR spectrum of compound $\mathbf{1}$ in $\mathrm{CD}_{3} \mathrm{OD}$ at $150 \mathrm{MHz}$, Detailed ${ }^{13} \mathrm{C}$ NMR spectrum of compound $\mathbf{1}$ in $\mathrm{CD}_{3} \mathrm{OD}$ at $150 \mathrm{MHz}$. spectrum of compound $\mathbf{1}$ in $\mathrm{CD}_{3} \mathrm{OD}$.

S10 $\quad \mathrm{HMBC}$ spectrum of compound $\mathbf{1}$ in $\mathrm{CD}_{3} \mathrm{OD}, \mathrm{COSY}$ spectrum of compound $\mathbf{1}$ in $\mathrm{CD}_{3} \mathrm{OD}$.

S11 NOESY spectrum of compound 1 in $\mathrm{CD}_{3} \mathrm{OD}$.

S12 ECD spectrum of 1

S13 HR-ESI-MS spectrum of compound 2, ${ }^{1} \mathrm{H}$ NMR spectrum of compound 2 in $\mathrm{CD}_{3} \mathrm{OD}$ at $600 \mathrm{MHz}$.

S14 Detailed ${ }^{1} \mathrm{H}$ NMR spectrum of compound 2 in $\mathrm{CD}_{3} \mathrm{OD}$ at $600 \mathrm{MHz}$, Detailed ${ }^{1} \mathrm{H}$ NMR spectrum of compound 2 in $\mathrm{CD}_{3} \mathrm{OD}$ at $600 \mathrm{MHz}$.

${ }^{13} \mathrm{C}$ NMR spectrum of compound 2 in $\mathrm{CD}_{3} \mathrm{OD}$ at $150 \mathrm{MHz}$, Detailed ${ }^{13} \mathrm{C}$ NMR spectrum of compound 2 in $\mathrm{CD}_{3} \mathrm{OD}$ at $150 \mathrm{MHz}$.

Detailed ${ }^{13} \mathrm{C}$ NMR spectrum of compound 2 in $\mathrm{CD}_{3} \mathrm{OD}$ at $150 \mathrm{MHz}$, HSQC spectrum of compound 2 in $\mathrm{CD}_{3} \mathrm{OD}$.

S17 $\quad \mathrm{HMBC}$ spectrum of compound $\mathbf{2}$ in $\mathrm{CD}_{3} \mathrm{OD}, \mathrm{COSY}$ spectrum of compound $\mathbf{2}$ in $\mathrm{CD}_{3} \mathrm{OD}$.

S18 NOESY spectrum of compound 2 in $\mathrm{CD}_{3} \mathrm{OD}$.

S19 HR-ESI-MS spectrum of compound 3, ${ }^{1} \mathrm{H}$ NMR spectrum of compound 3 in $\mathrm{CD}_{3} \mathrm{OD}$ at $600 \mathrm{MHz}$. NMR spectrum of compound 3 in $\mathrm{CD}_{3} \mathrm{OD}$ at $600 \mathrm{MHz}$. 
${ }^{3} \mathrm{C}$ NMR spectrum of compound 3 in $\mathrm{CD}_{3} \mathrm{OD}$ at $150 \mathrm{MHz}$, Detailed ${ }^{13} \mathrm{C}$ NMR spectrum of compound 3 in $\mathrm{CD}_{3} \mathrm{OD}$ at $150 \mathrm{MHz}$.

S22

Detailed ${ }^{13} \mathrm{C}$ NMR spectrum of compound 3 in $\mathrm{CD}_{3} \mathrm{OD}$ at $150 \mathrm{MHz}$, HSQC spectrum of compound 3 in $\mathrm{CD}_{3} \mathrm{OD}$.

$\mathrm{HMBC}$ spectrum of compound $\mathbf{3}$ in $\mathrm{CD}_{3} \mathrm{OD}$, COSY spectrum of compound $\mathbf{3}$ in $\mathrm{CD}_{3} \mathrm{OD}$.

S24 NOESY spectrum of compound 3 in $\mathrm{CD}_{3} \mathrm{OD}$.

S25 HR-ESI-MS spectrum of compound $4,{ }^{1} \mathrm{H}$ NMR spectrum of compound 4 in $\mathrm{CD}_{3} \mathrm{OD}$ at $600 \mathrm{MHz}$.

S26 Detailed ${ }^{1} \mathrm{H}$ NMR spectrum of compound 4 in $\mathrm{CD}_{3} \mathrm{OD}$ at $600 \mathrm{MHz}$, Detailed ${ }^{1} \mathrm{H}$ NMR spectrum of compound 4 in $\mathrm{CD}_{3} \mathrm{OD}$ at $600 \mathrm{MHz}$.

S27 ${ }^{13} \mathrm{C}$ NMR spectrum of compound 4 in $\mathrm{CD}_{3} \mathrm{OD}$ at $150 \mathrm{MHz}$, Detailed ${ }^{13} \mathrm{C}$ NMR spectrum of compound 4 in $\mathrm{CD}_{3} \mathrm{OD}$ at $150 \mathrm{MHz}$.

S28 Detailed ${ }^{13} \mathrm{C}$ NMR spectrum of compound 4 in $\mathrm{CD}_{3} \mathrm{OD}$ at $150 \mathrm{MHz}$, HSQC spectrum of compound 4 in $\mathrm{CD}_{3} \mathrm{OD}$.

S29 $\mathrm{HMBC}$ spectrum of compound 4 in $\mathrm{CD}_{3} \mathrm{OD}, \mathrm{COSY}$ spectrum of compound 4 in $\mathrm{CD}_{3} \mathrm{OD}$.

S30 NOESY spectrum of compound 4 in $\mathrm{CD}_{3} \mathrm{OD}$.

S31 HR-ESI-MS spectrum of compound $\mathbf{5},{ }^{1} \mathrm{H}$ NMR spectrum of compound $\mathbf{5}$ in $\mathrm{CD}_{3} \mathrm{OD}$ at $600 \mathrm{MHz}$.

S32 Detailed ${ }^{1} \mathrm{H}$ NMR spectrum of compound 5 in $\mathrm{CD}_{3} \mathrm{OD}$ at $600 \mathrm{MHz}$, Detailed ${ }^{1} \mathrm{H}$ NMR spectrum of compound 5 in $\mathrm{CD}_{3} \mathrm{OD}$ at $600 \mathrm{MHz}$.

S33 ${ }^{13} \mathrm{C}$ NMR spectrum of compound 5 in $\mathrm{CD}_{3} \mathrm{OD}$ at $150 \mathrm{MHz}$, Detailed ${ }^{13} \mathrm{C}$ NMR spectrum of compound 5 in $\mathrm{CD}_{3} \mathrm{OD}$ at $125 \mathrm{MHz}$.

S34 Detailed ${ }^{13} \mathrm{C}$ NMR spectrum of compound 5 in $\mathrm{CD}_{3} \mathrm{OD}$ at $150 \mathrm{MHz}$, HSQC spectrum of compound 5 in $\mathrm{CD}_{3} \mathrm{OD}$.

S35 HMBC spectrum of compound 5 in $\mathrm{CD}_{3} \mathrm{OD}, \mathrm{COSY}$ spectrum of compound $\mathbf{5}$ in $\mathrm{CD}_{3} \mathrm{OD}$.

S36 NOESY spectrum of compound 5 in $\mathrm{CD}_{3} \mathrm{OD}$. 
Table S1 ${ }^{13} \mathrm{C}-\mathrm{NMR}(150 \mathrm{MHz})$ spectroscopic data for compounds 6-10 in $\mathrm{CD}_{3} \mathrm{OD}$.

\begin{tabular}{|c|c|c|c|c|c|}
\hline NO. & 6 & 7 & 8 & 9 & 10 \\
\hline 1 & 210.3 & 212.5 & 204.1 & 205.6 & 206.0 \\
\hline 2 & 41.3 & 42.2 & 128.7 & 129.6 & 128.7 \\
\hline 3 & 73.6 & 70.5 & 141.7 & 142.3 & 142.5 \\
\hline 4 & 36.5 & 78.6 & 36.0 & 37.2 & 37.1 \\
\hline 5 & 61.5 & 65.4 & 77.2 & 78.5 & 78.1 \\
\hline 6 & 62.4 & 59.7 & 73.4 & 75.1 & 75.4 \\
\hline 7 & 25.1 & 26.7 & 26.8 & 28.8 & 30.8 \\
\hline 8 & 35.7 & 34.8 & 35.4 & 37.5 & 37.7 \\
\hline 9 & 38.6 & 39.8 & 36.3 & 37.4 & 38.2 \\
\hline 10 & 52.1 & 52.0 & 52.6 & 52.6 & 52.5 \\
\hline 11 & 22.2 & 21.8 & 23.2 & 24.1 & 22.9 \\
\hline 12 & 37.9 & 30.3 & 38.3 & 40.8 & 40.7 \\
\hline 13 & 52.3 & 55.0 & 52.2 & 53.2 & 54.0 \\
\hline 14 & 81.3 & 81.2 & 82.4 & 82.4 & 84.0 \\
\hline 15 & 84.4 & 33.0 & 83.6 & 83.4 & 76.5 \\
\hline 16 & 122.1 & 37.9 & 120.4 & 127.3 & 127.8 \\
\hline 17 & 162.6 & 86.7 & 162.2 & 158.9 & 154.6 \\
\hline 18 & 16.8 & 20.8 & 16.1 & 17.9 & 19.9 \\
\hline 19 & 14.3 & 15.2 & 15.0 & 16.0 & 16.7 \\
\hline 20 & 35.0 & 79.3 & 36.1 & 35.5 & 35.2 \\
\hline 21 & 17.8 & 19.0 & 17.2 & 19.8 & 18.6 \\
\hline 22 & 78.9 & 81.6 & 78.6 & 79.7 & 79.4 \\
\hline 23 & 32.1 & 33.9 & 32.4 & 32.9 & 32.7 \\
\hline 24 & 150.8 & 151.0 & 150.8 & 150.1 & 150.5 \\
\hline 25 & 121.8 & 121.7 & 121.2 & 122.2 & 122.0 \\
\hline 26 & 167.7 & 169.6 & 167.4 & 166.8 & 166.7 \\
\hline 27 & 12.5 & 11.2 & 11.6 & 13.0 & 13.0 \\
\hline 28 & 19.7 & 20.4 & 20.6 & 18.5 & 20.4 \\
\hline $\mathrm{CH}_{3} \mathrm{CO}-1^{\prime}$ & 170.1 & - & 170.6 & - & - \\
\hline $\mathrm{CH}_{3} \mathrm{CO}-2^{\prime}$ & 20.9 & - & 21.9 & - & - \\
\hline $\mathrm{OMe}$ & 53.7 & - & - & - & - \\
\hline
\end{tabular}


Table S2 ${ }^{1} \mathrm{H}-\mathrm{NMR}(600 \mathrm{MHz})$ spectroscopic data for compounds 6-10 in $\mathrm{CD}_{3} \mathrm{OD}$.

\begin{tabular}{|c|c|c|c|c|c|}
\hline NO. & 6 & 7 & 8 & 9 & 10 \\
\hline 2 & $\begin{array}{l}\alpha: 2.88 \mathrm{dd}(16.2,3.0) \\
\beta: 2.94 \mathrm{dd}(16.2,7.8)\end{array}$ & $\begin{array}{l}\alpha: 2.55 \mathrm{dd}(15.6,3.2) \\
\beta: 2.91 \mathrm{dd}(15.6,7.2)\end{array}$ & $5.87 \mathrm{dd}(10.2,3.6)$ & $6.15 \mathrm{dd}(10.2,3.6)$ & $6.18 \mathrm{dd}(10.2,3.6)$ \\
\hline 3 & $3.79 \mathrm{~m}$ & $4.08 \mathrm{~m}$ & $\begin{array}{c}6.57 \text { ddd }(10.2, \\
5.4,3.6)\end{array}$ & $\begin{array}{c}6.67 \text { ddd }(10.2 \\
5.4,3.6)\end{array}$ & $\begin{array}{c}6.69 \text { ddd }(10.2, \\
5.4,3.6)\end{array}$ \\
\hline \multirow{4}{*}{4} & & \multirow{4}{*}{$3.21 \mathrm{~d}(3.2)$} & $\alpha: 2.10 \mathrm{dd}(10.2$ & $\alpha: 2.45$ dd (10.2, & $\alpha: 2.45 \mathrm{dd}(10.2$ \\
\hline & $\alpha: 1.68$ (br d, 16.2) & & 5.4) & 5.4) & $5.4)$ \\
\hline & $\beta: 3.66 \mathrm{~d}(3.0)$ & & $\beta: 3.36 \mathrm{dt}(16.2$ & $\beta: 3.76 \mathrm{dt}(16.2$ & $\beta: 3.74 \mathrm{dt}(16.2$ \\
\hline & & & 3.6) & 3.6) & $3.6)$ \\
\hline 6 & 3.40 br s & $3.30 \mathrm{br} \mathrm{s}$ & $3.60 \mathrm{t}(3.6)$ & 4.30 br s & 4.32 br s \\
\hline 7 & $\alpha: 1.62 \mathrm{~m}$ & $\alpha: 2.49 \mathrm{~m}$ & $\alpha: 1.97 \mathrm{~m}$ & $\alpha: 2.97 \mathrm{~m}$ & $\alpha: 2.77 \mathrm{~m}$ \\
\hline & $\beta: 2.49 \mathrm{~m}$ & $\beta: 2.60 \mathrm{~m}$ & $\beta: 2.21 \mathrm{~m}$ & $\beta: 3.47 \mathrm{~m}$ & $\beta: 3.57 \mathrm{~m}$ \\
\hline 8 & $2.59 \mathrm{~m}$ & $2.63 \mathrm{~m}$ & $2.30 \mathrm{~m}$ & $2.86 \mathrm{~m}$ & $2.76 \mathrm{~m}$ \\
\hline 9 & $1.93 \mathrm{~m}$ & $2.57 \mathrm{~m}$ & $2.12 \mathrm{~m}$ & $3.79 \mathrm{~m}$ & $3.69 \mathrm{~m}$ \\
\hline 11 & $1.25 \mathrm{~m}, 1.38 \mathrm{~m}$ & $1.48 \mathrm{~m}, 1.70 \mathrm{~m}$ & $2.16 \mathrm{~m}, 2.12 \mathrm{~m}$ & $1.64 \mathrm{~m}, 2.82 \mathrm{~m}$ & $1.42 \mathrm{~m}, 2.62 \mathrm{~m}$ \\
\hline 12 & $1.74 \mathrm{~m}, 1.47 \mathrm{~m}$ & $1.51 \mathrm{~m}, 2.21 \mathrm{~m}$ & $1.82 \mathrm{~m}, 2.31 \mathrm{~m}$ & $1.95 \mathrm{~m}, 2.34 \mathrm{~m}$ & $1.95 \mathrm{~m}, 2.34 \mathrm{~m}$ \\
\hline 15 & $5.82 \mathrm{~d}(3.0)$ & $5.04 \mathrm{~d}(3.2)$ & $5.37 \mathrm{~d}(2.4)$ & $5.10 \mathrm{~d}(2.4)$ & $5.25 \mathrm{~d}(2.4)$ \\
\hline 16 & $5.78 \mathrm{~d}(3.0)$ & $3.67 \mathrm{~m}, 3.76 \mathrm{~m}$ & $5.60 \mathrm{~d}(2.4)$ & $6.13 \mathrm{~d}(2.4)$ & 5.71 br s \\
\hline 18 & $1.37 \mathrm{~s}$ & $1.21 \mathrm{~s}$ & $1.16 \mathrm{~s}$ & $1.43 \mathrm{~s}$ & $1.39 \mathrm{~s}$ \\
\hline 19 & $1.35 \mathrm{~s}$ & $1.26 \mathrm{~s}$ & $1.21 \mathrm{~s}$ & $1.72 \mathrm{~s}$ & $1.70 \mathrm{~s}$ \\
\hline 20 & $2.57 \mathrm{~m}$ & - & 2.53 & $2.64 \mathrm{~m}$ & $2.62 \mathrm{~m}$ \\
\hline 21 & $1.21 \mathrm{~d}(7.2)$ & $1.07 \mathrm{~s}$ & $1.12 \mathrm{~d}(7.2)$ & $1.22 \mathrm{~d}(7.2)$ & $1.26 \mathrm{~d}(7.2)$ \\
\hline 22 & $4.39 \mathrm{dd}(12.0,3.6)$ & $5.08 \mathrm{dd}(12.0,3.6)$ & $4.34 \mathrm{dd}(12.0,3.6)$ & $4.45 \mathrm{dd}(12.0,3.6)$ & $4.43 \mathrm{dd}(12.0,3.6)$ \\
\hline 23 & $2.29 \mathrm{~m}, 2.59 \mathrm{~m}$ & $1.86 \mathrm{~m}, 1.96 \mathrm{~m}$ & $2.28 \mathrm{~m}, 2.39 \mathrm{~m}$ & $2.08 \mathrm{~m}, 2.49 \mathrm{~m}$ & $2.06 \mathrm{~m}, 2.52 \mathrm{~m}$ \\
\hline 27 & $1.84 \mathrm{~s}$ & $1.86 \mathrm{~s}$ & $1.86 \mathrm{~s}$ & $1.85 \mathrm{~s}$ & $1.85 \mathrm{~s}$ \\
\hline 28 & $1.53(\mathrm{br} \mathrm{s})$ & $1.99 \mathrm{~s}$ & $1.99 \mathrm{~s}$ & $1.52 \mathrm{~s}$ & $1.50 \mathrm{~s}$ \\
\hline $\mathrm{CH}_{3} \mathrm{CO}$ & $2.09 \mathrm{~s}$ & $2.10 \mathrm{~s}$ & $2.03 \mathrm{~s}$ & - & - \\
\hline $\mathrm{OMe}$ & $3.21 \mathrm{~s}$ & - & - & - & - \\
\hline
\end{tabular}

Chemical shifts are in ppm, and coupling constants $(J)$ in $\mathrm{Hz}$ are given in parentheses. 
HR-ESI-MS spectrum of compound 1

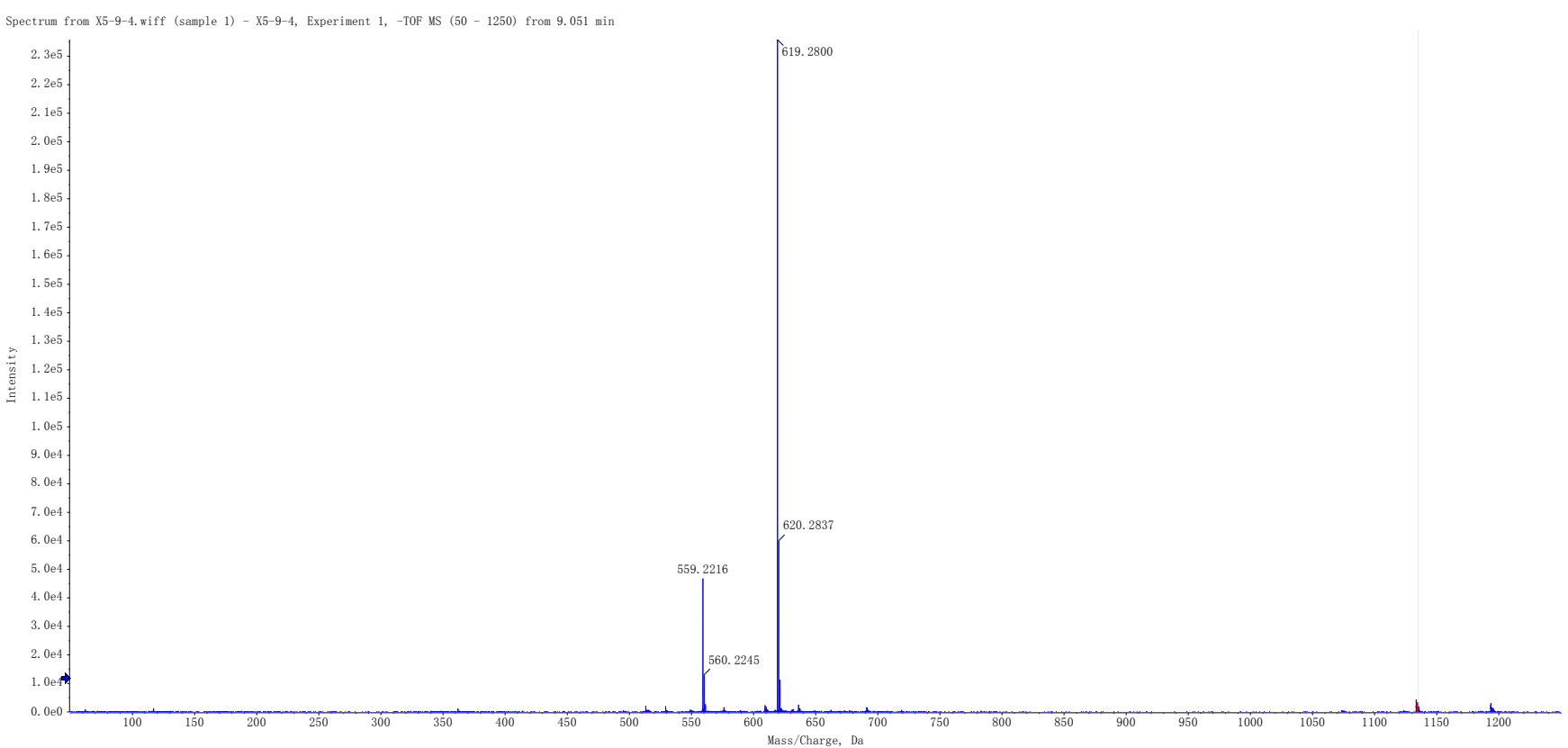

${ }^{1} \mathrm{H}$ NMR spectrum of compound $1 \mathrm{CD}_{3} \mathrm{OD}$ at $600 \mathrm{MHz}$

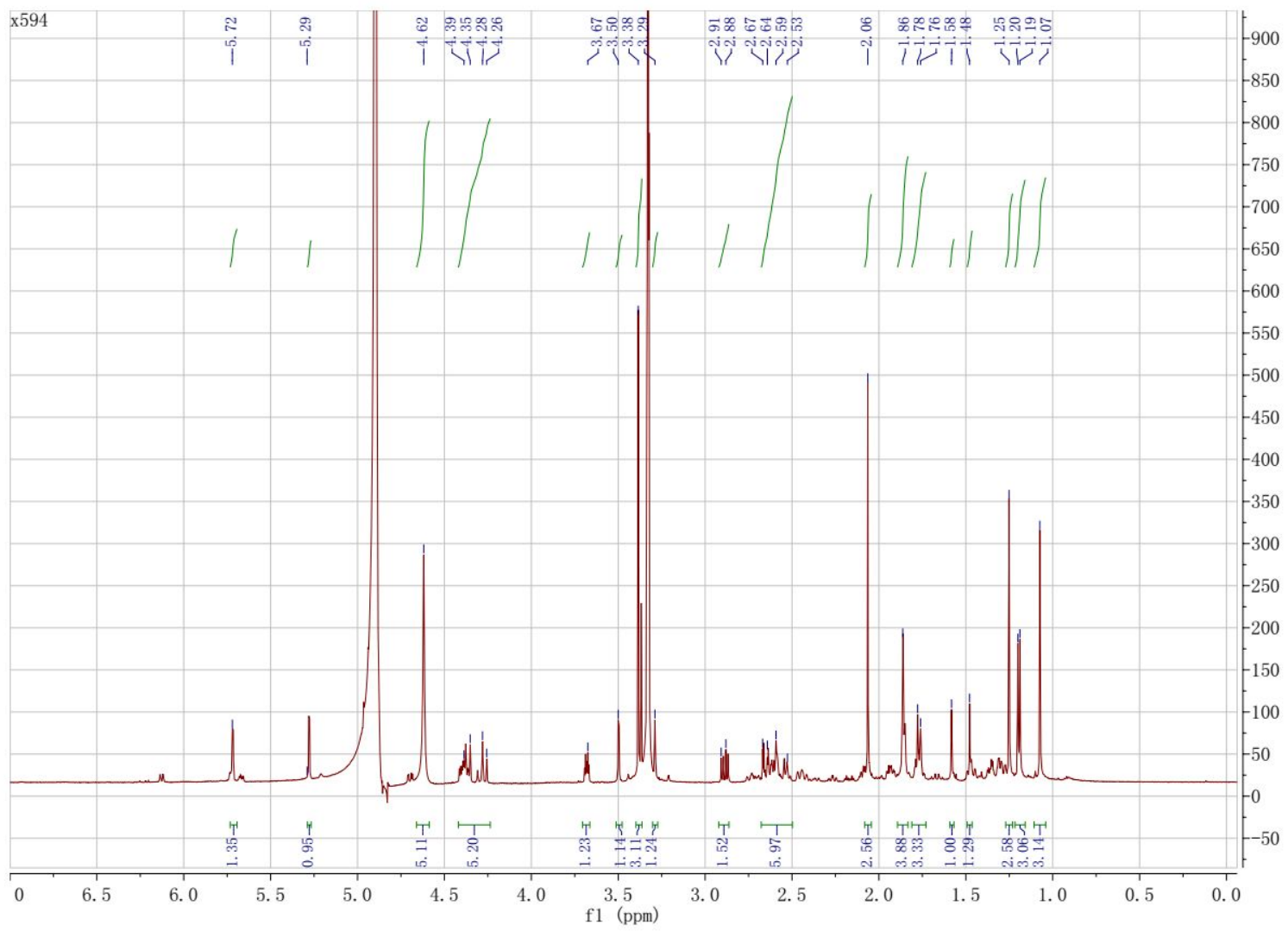


Detailed ${ }^{1} \mathrm{H}$ NMR spectrum of compound 1 in $\mathrm{CD}_{3} \mathrm{OD}$ at $600 \mathrm{MHz}$

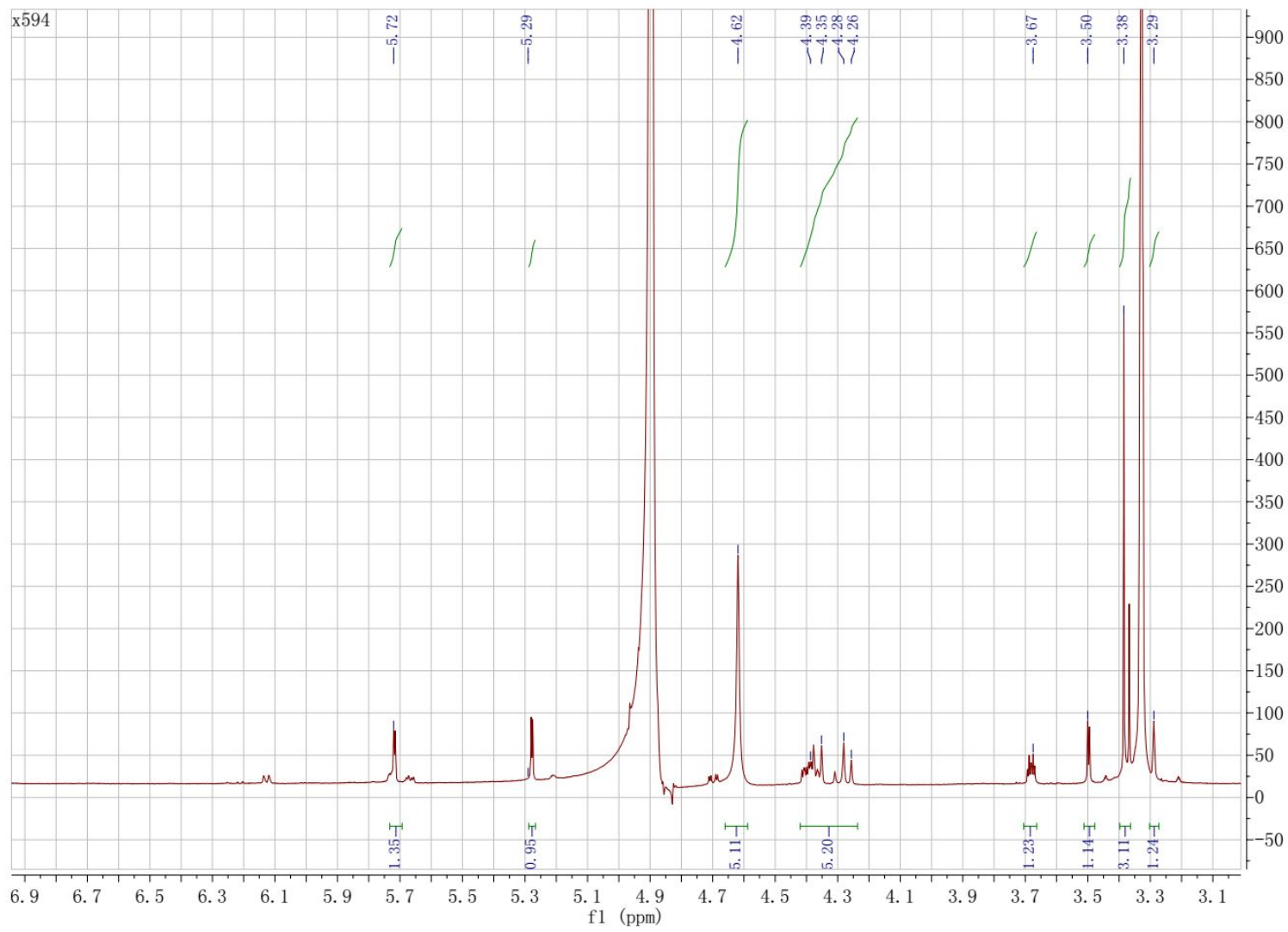

Detailed ${ }^{1} \mathrm{H}$ NMR spectrum of compound 1 in $\mathrm{CD}_{3} \mathrm{OD}$ at $600 \mathrm{MHz}$

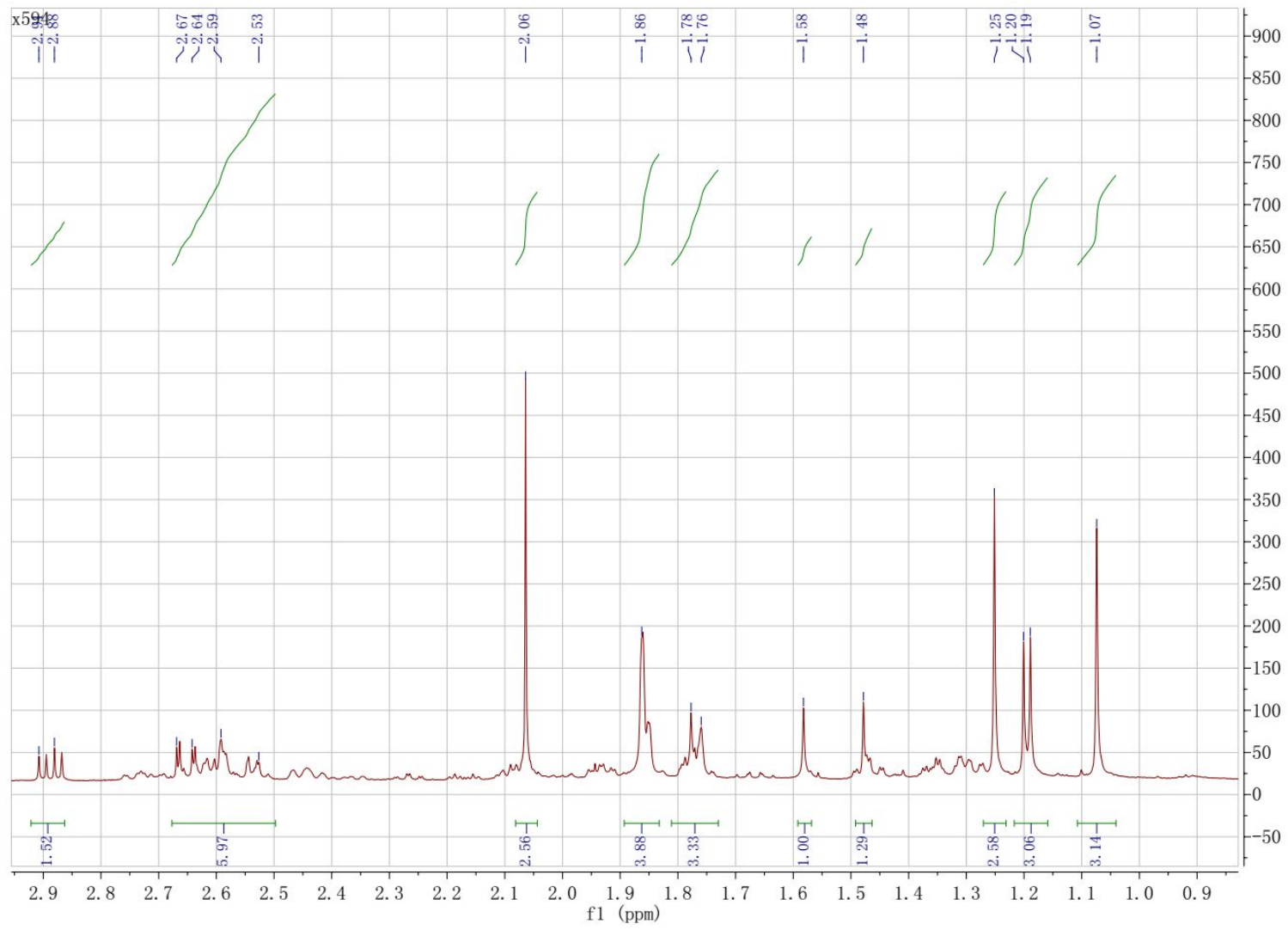


${ }^{13} \mathrm{C}$ NMR spectrum of compound 1 in $\mathrm{CD}_{3} \mathrm{OD}$ at $150 \mathrm{MHz}$

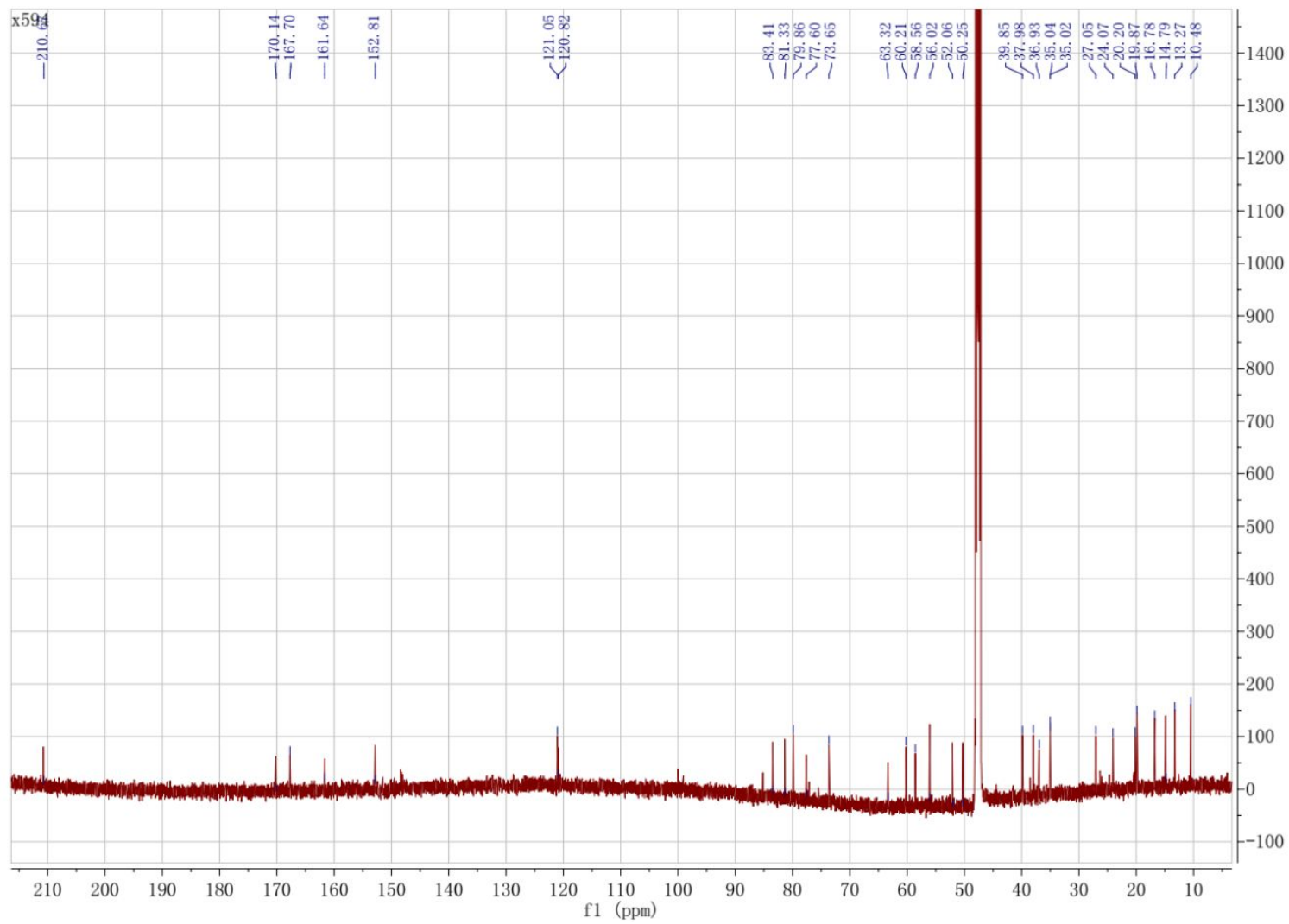

Detailed ${ }^{13} \mathrm{C}$ NMR spectrum of compound 1 in $\mathrm{CD}_{3} \mathrm{OD}$ at $150 \mathrm{MHz}$

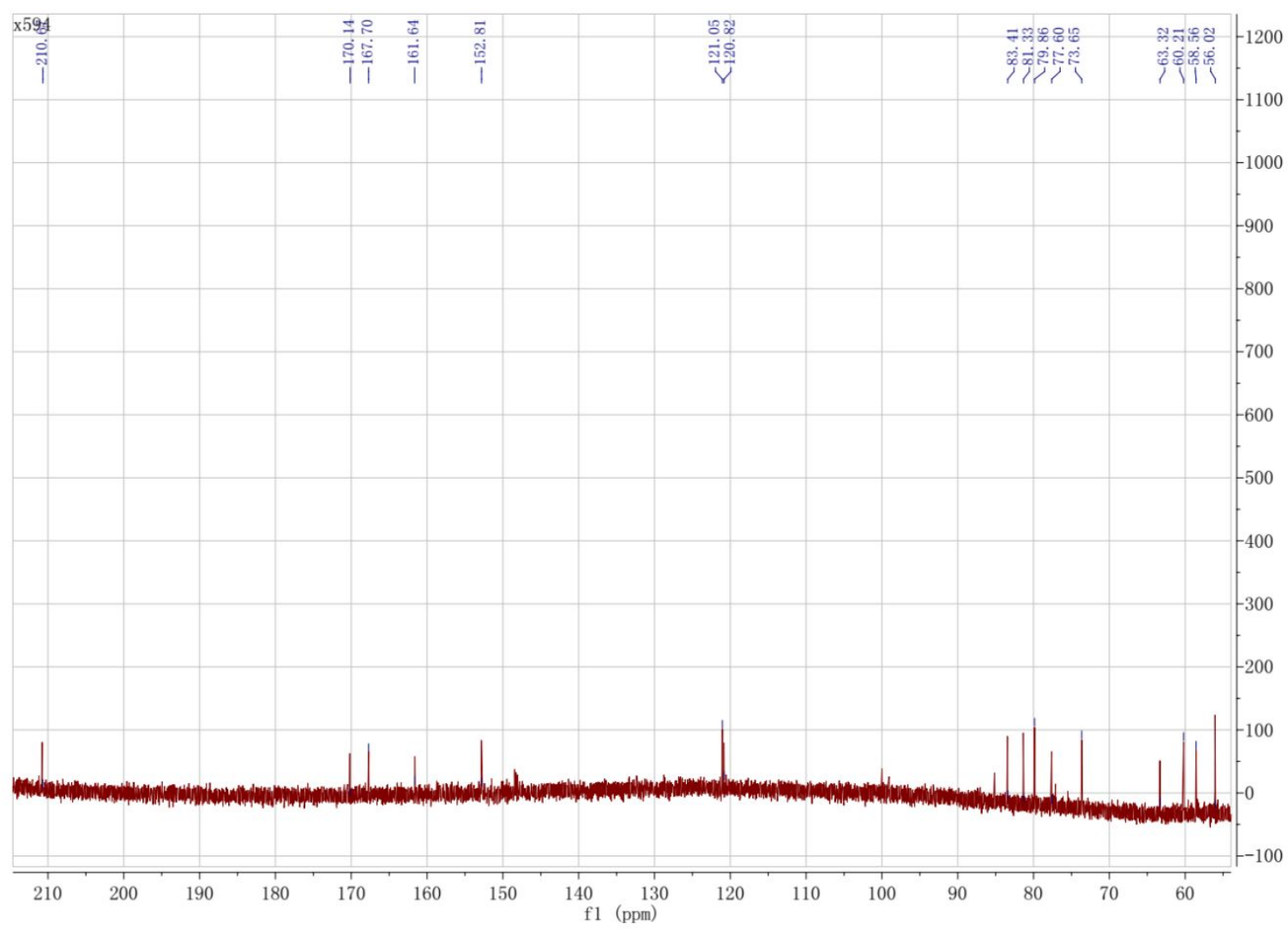


Detailed ${ }^{13} \mathrm{C}$ NMR spectrum of compound 1 in $\mathrm{CD}_{3} \mathrm{OD}$ at $150 \mathrm{MHz}$

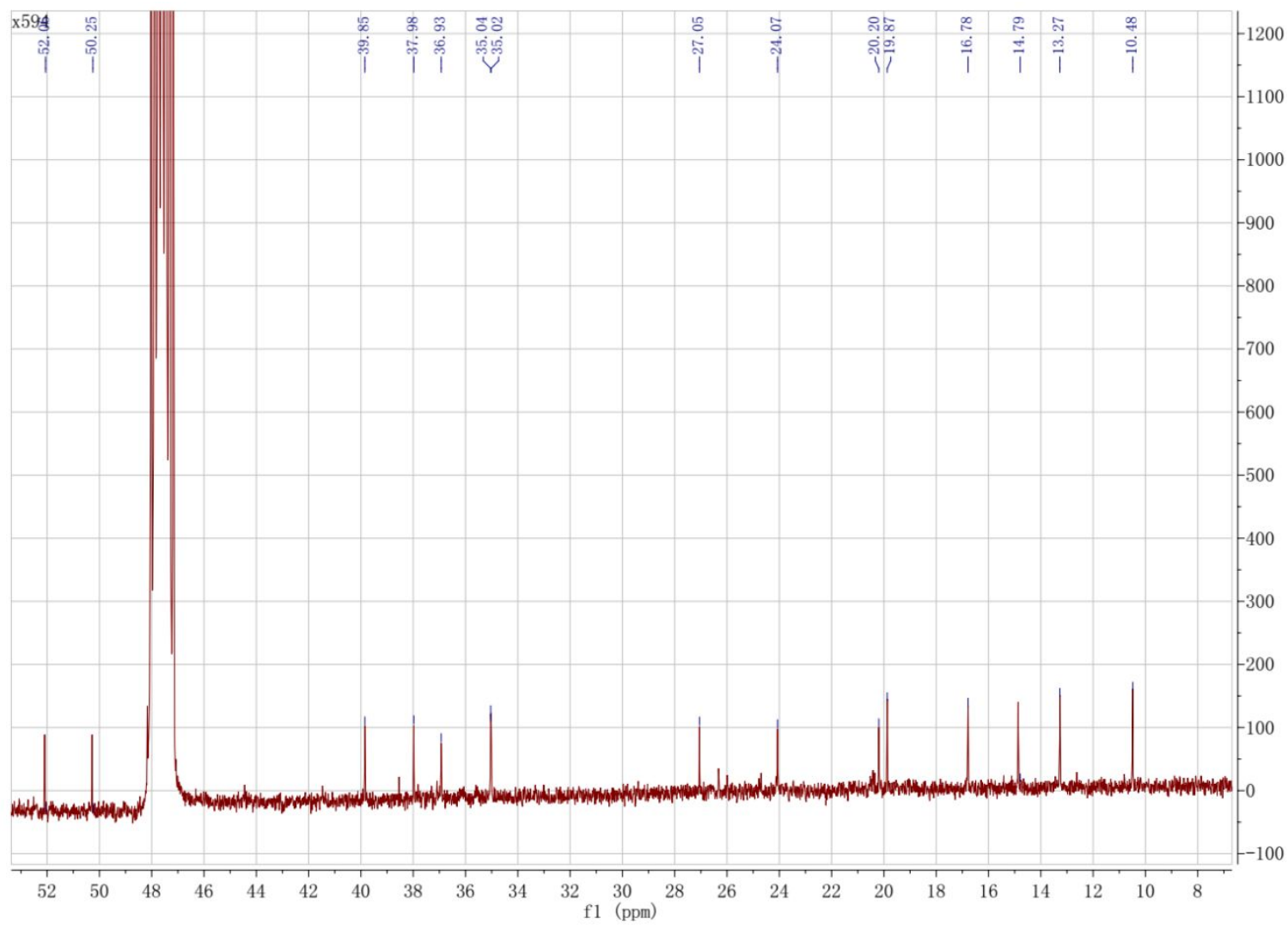

HSQC spectrum of compound 1 in $\mathrm{CD}_{3} \mathrm{OD}$

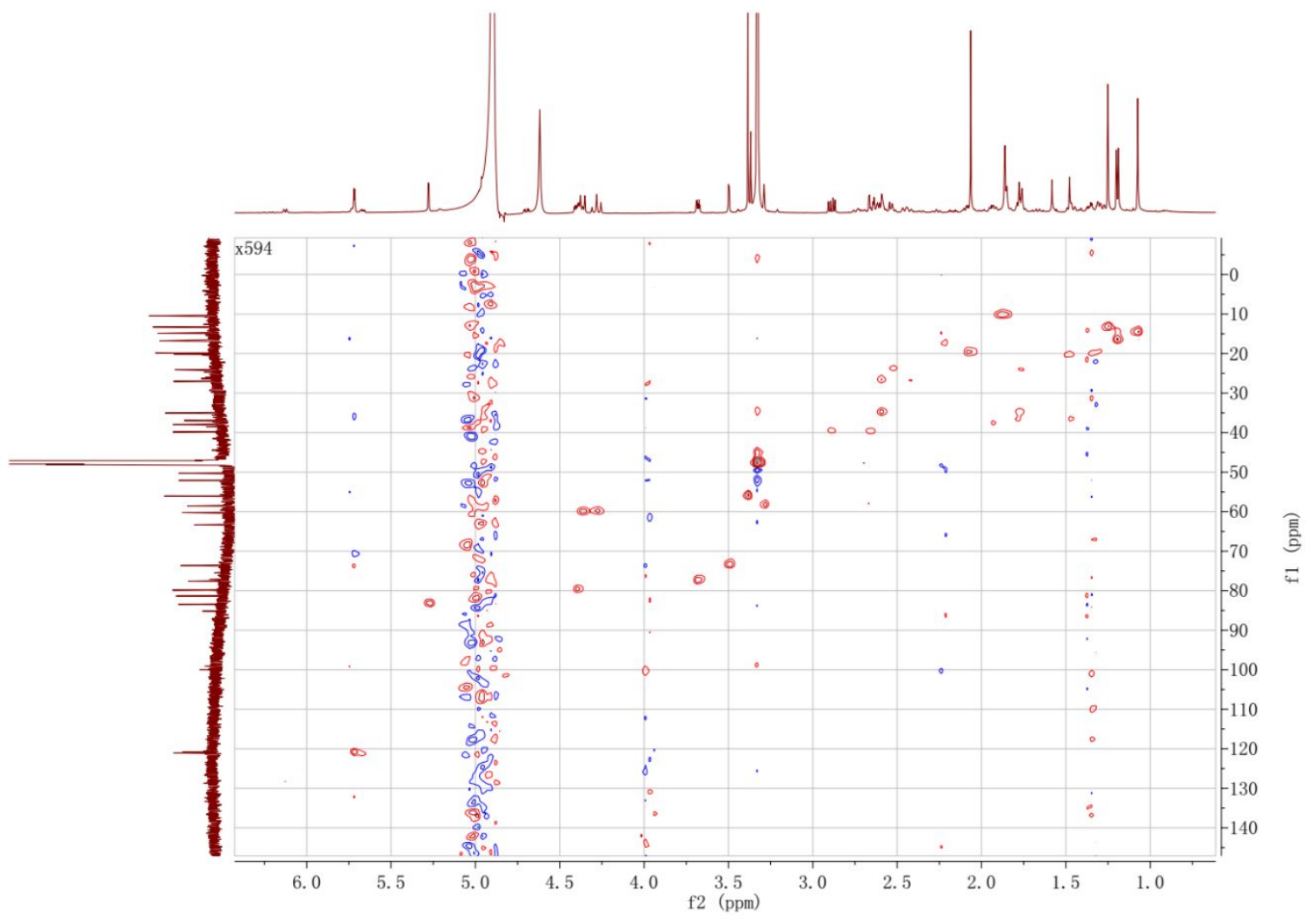


$\mathrm{HMBC}$ spectrum of compound $\mathbf{1}$ in $\mathrm{CD}_{3} \mathrm{OD}$

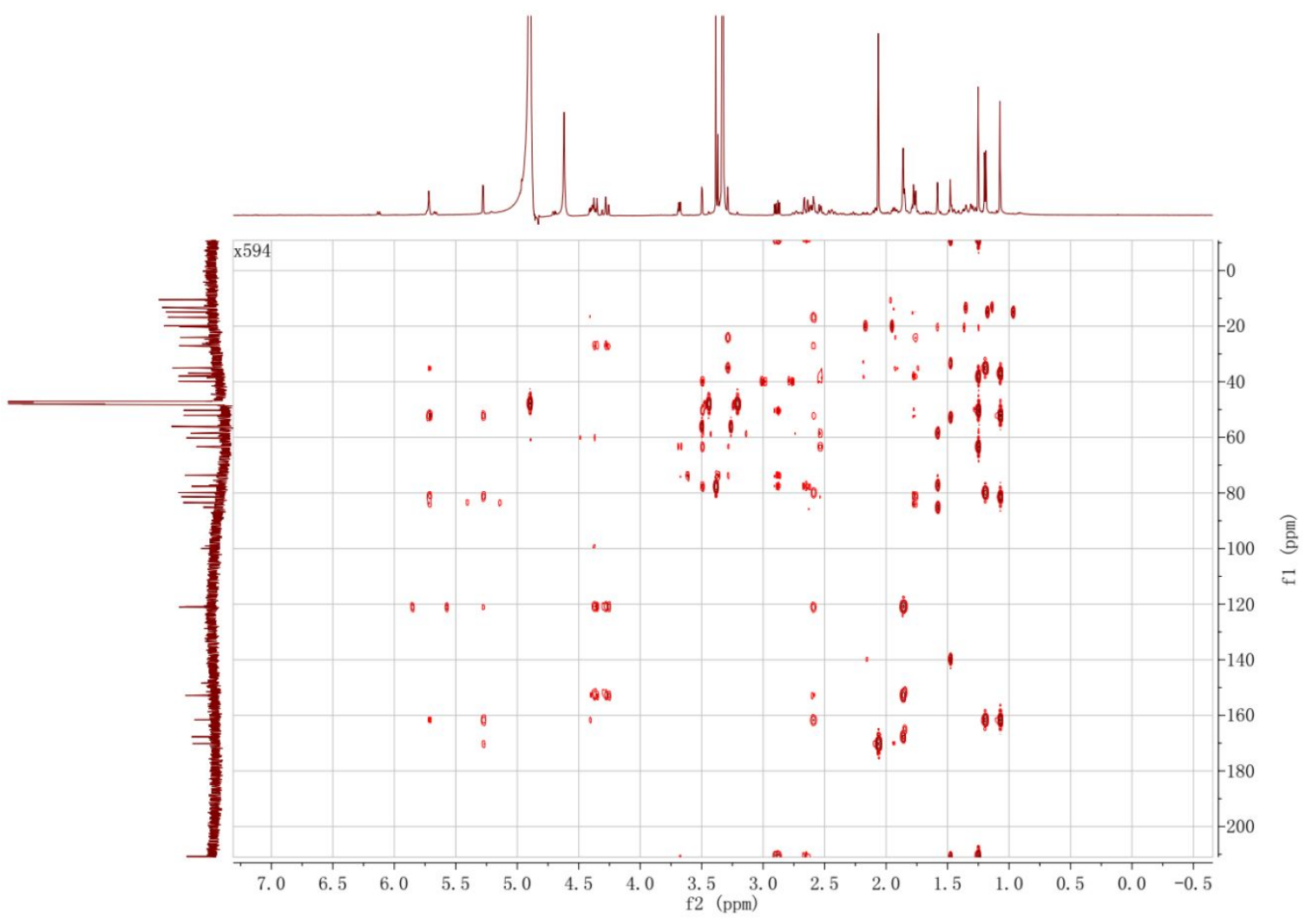

COSY spectrum of compound 1 in $\mathrm{CD}_{3} \mathrm{OD}$

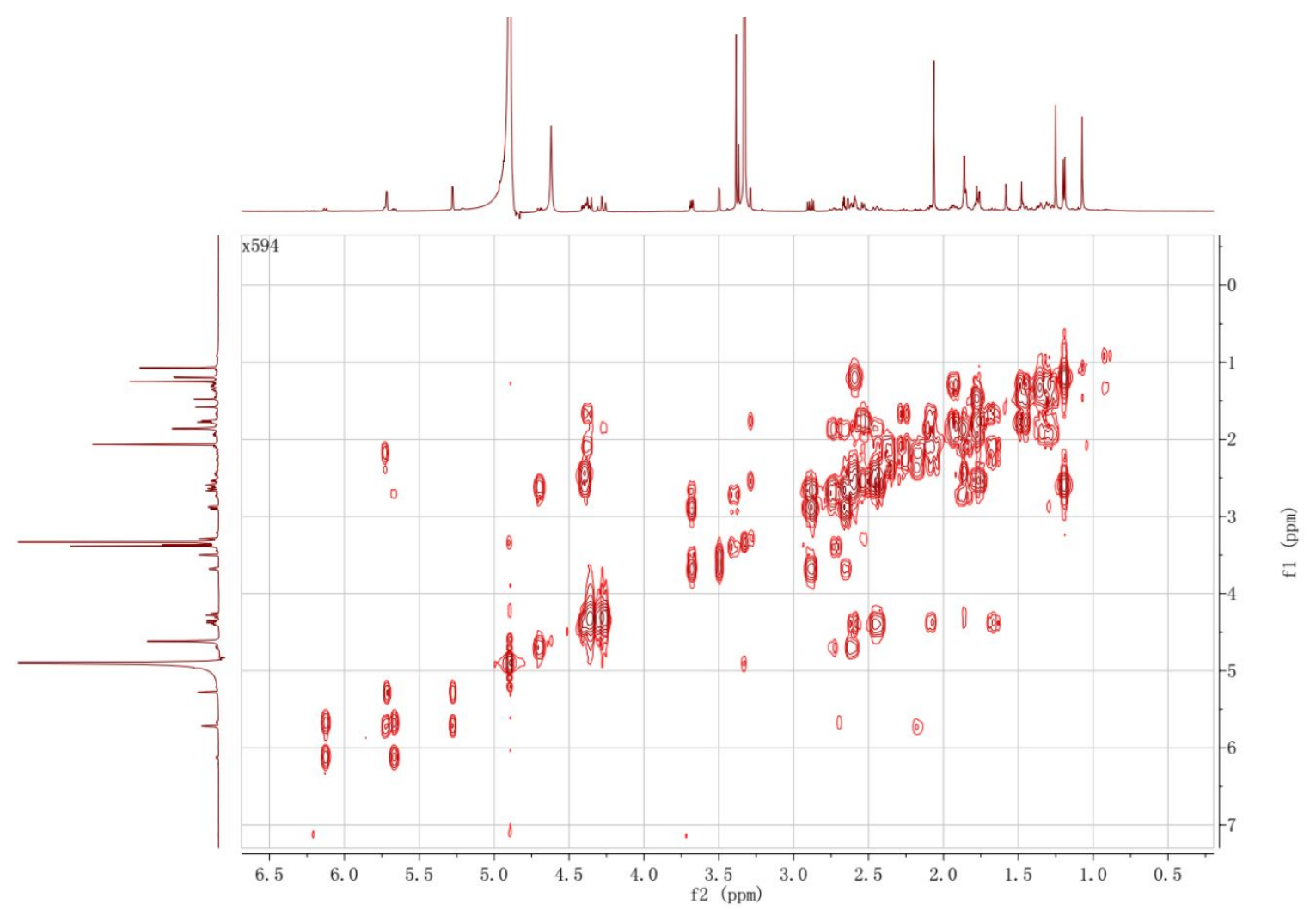


NOESY spectrum of compound 1 in $\mathrm{CD}_{3} \mathrm{OD}$

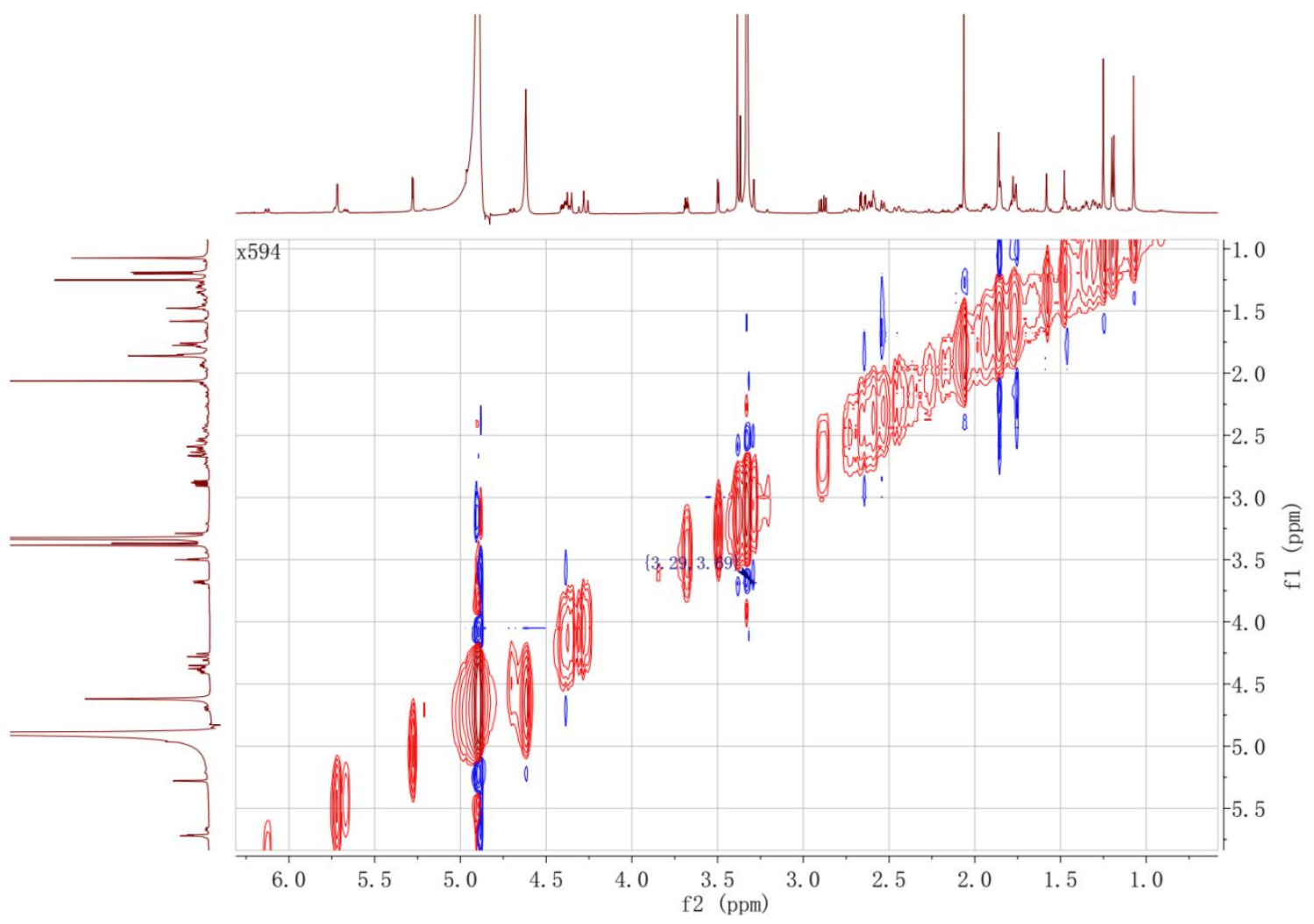




\section{ECD spectrum of $\mathbf{1}$}

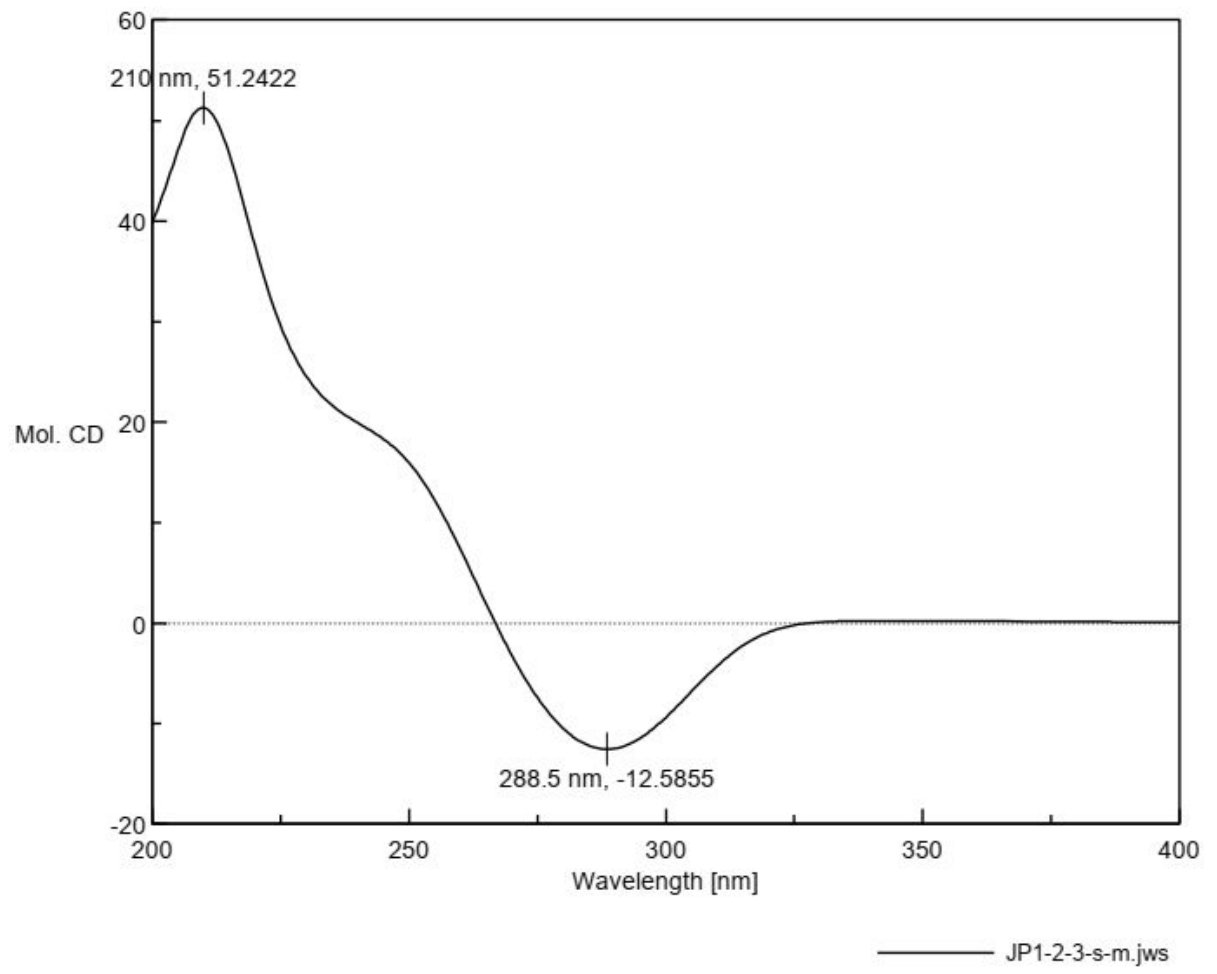

$\begin{array}{ll}\text { [Measurement Information] } \\ \text { Instrument Name } & \mathrm{J}-815 \\ \text { Model Name } & \mathrm{J}-815 \\ \text { Serial No. } & \mathrm{A} 024461168 \\ & \\ \text { Accessory } & \text { Standard } \\ \text { Accessory S/N } & \text { A024461168 } \\ \text { Cell Length } & 1 \mathrm{~mm} \\ & \\ \text { Measurement date } & 2020 / 3 / 1911: 25 \\ & \\ \text { Photometric Mode } & \mathrm{CD}, \mathrm{HT}, \mathrm{Abs} \\ \text { Measure Range } & 400-200 \mathrm{~nm} \\ \text { Data pitch } & 0.5 \mathrm{~nm} \\ \text { Sensitivity } & \text { Standard } \\ \text { D.I.T. } & 1 \mathrm{sec} \\ \text { Bandwidth } & 1.00 \mathrm{~nm} \\ \text { Start Mode } & \text { Immediately } \\ \text { Scanning Speed } & 100 \mathrm{~nm} / \mathrm{min} \\ \text { Baseline Correction } & \text { Baseline } \\ \text { Shutter Control } & \text { Auto } \\ \text { CD Detector } & \text { PMT } \\ \text { PMT Voltage } & \text { Auto } \\ \text { Accumulations } & 2 \\ \text { Solvent } & \text { MEOH } \\ \text { Concentration } & 0.4 \text { (w/v)\% }\end{array}$


HR-ESI-MS spectrum of compound 2

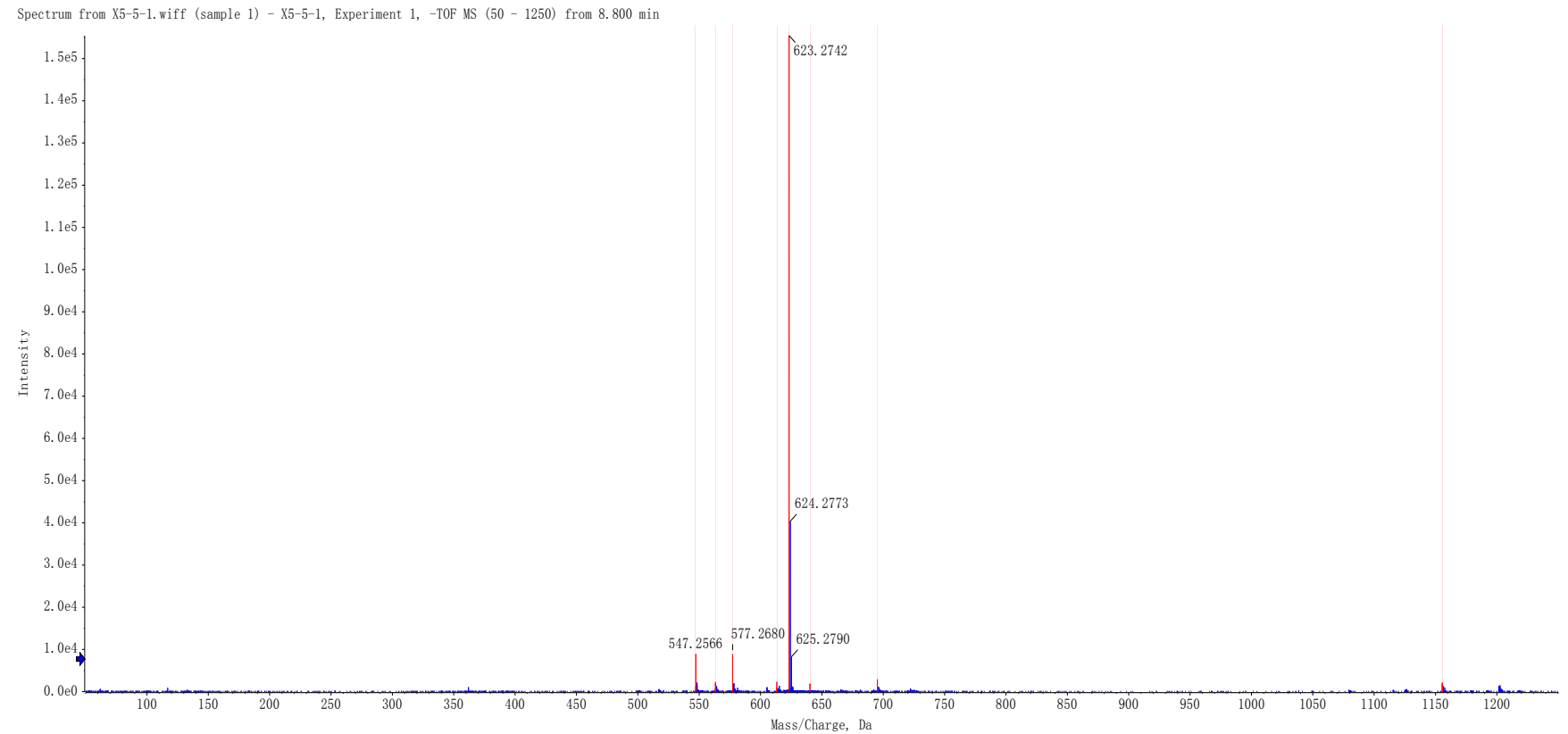

${ }^{1} \mathrm{H}$ NMR spectrum of compound 2 in $\mathrm{CD}_{3} \mathrm{OD}$ at $600 \mathrm{MHz}$

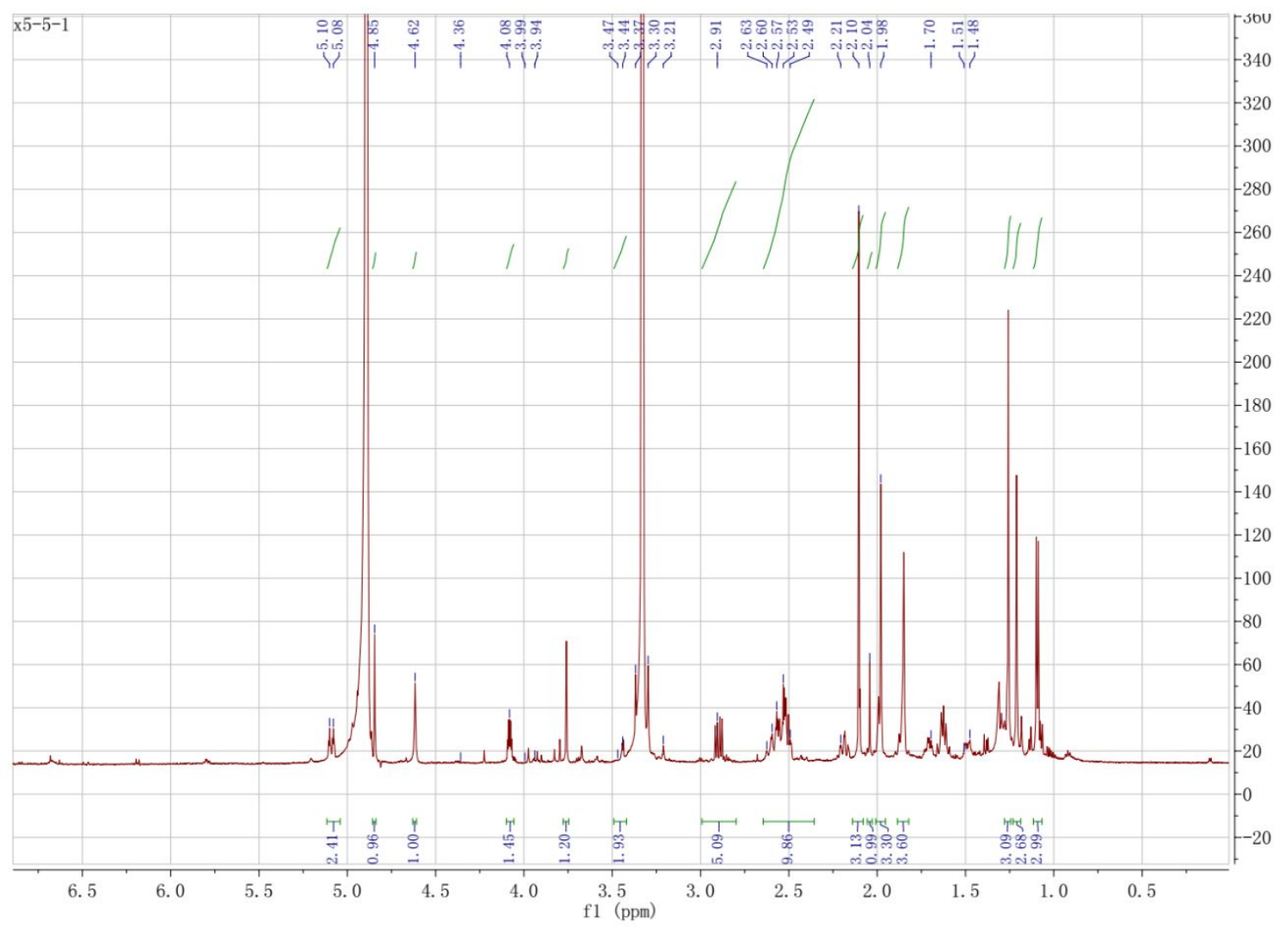


Detailed ${ }^{1} \mathrm{H}$ NMR spectrum of compound 2 in $\mathrm{CD}_{3} \mathrm{OD}$ at $600 \mathrm{MHz}$

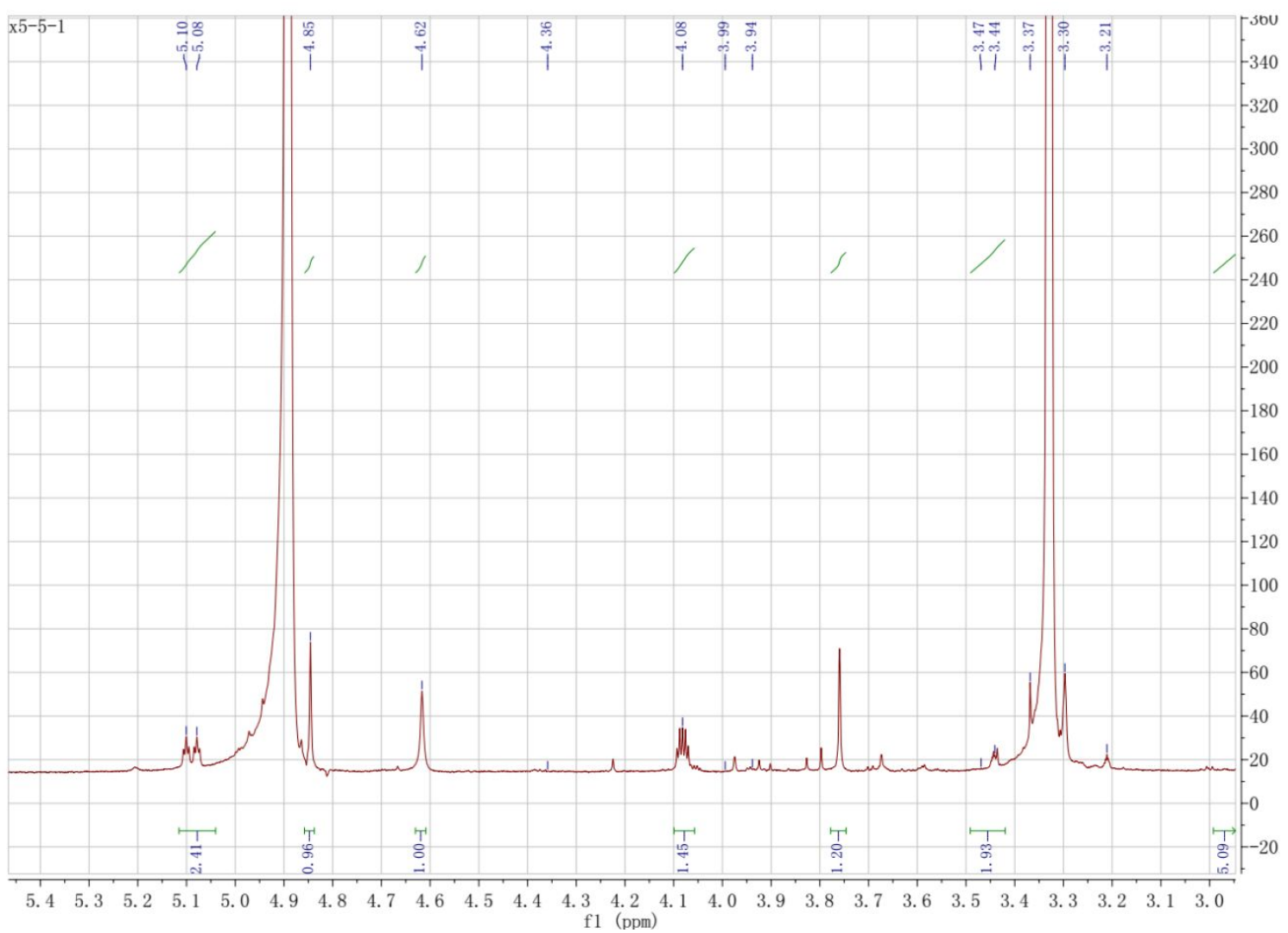

Detailed ${ }^{1} \mathrm{H}$ NMR spectrum of compound 2 in $\mathrm{CD}_{3} \mathrm{OD}$ at $600 \mathrm{MHz}$

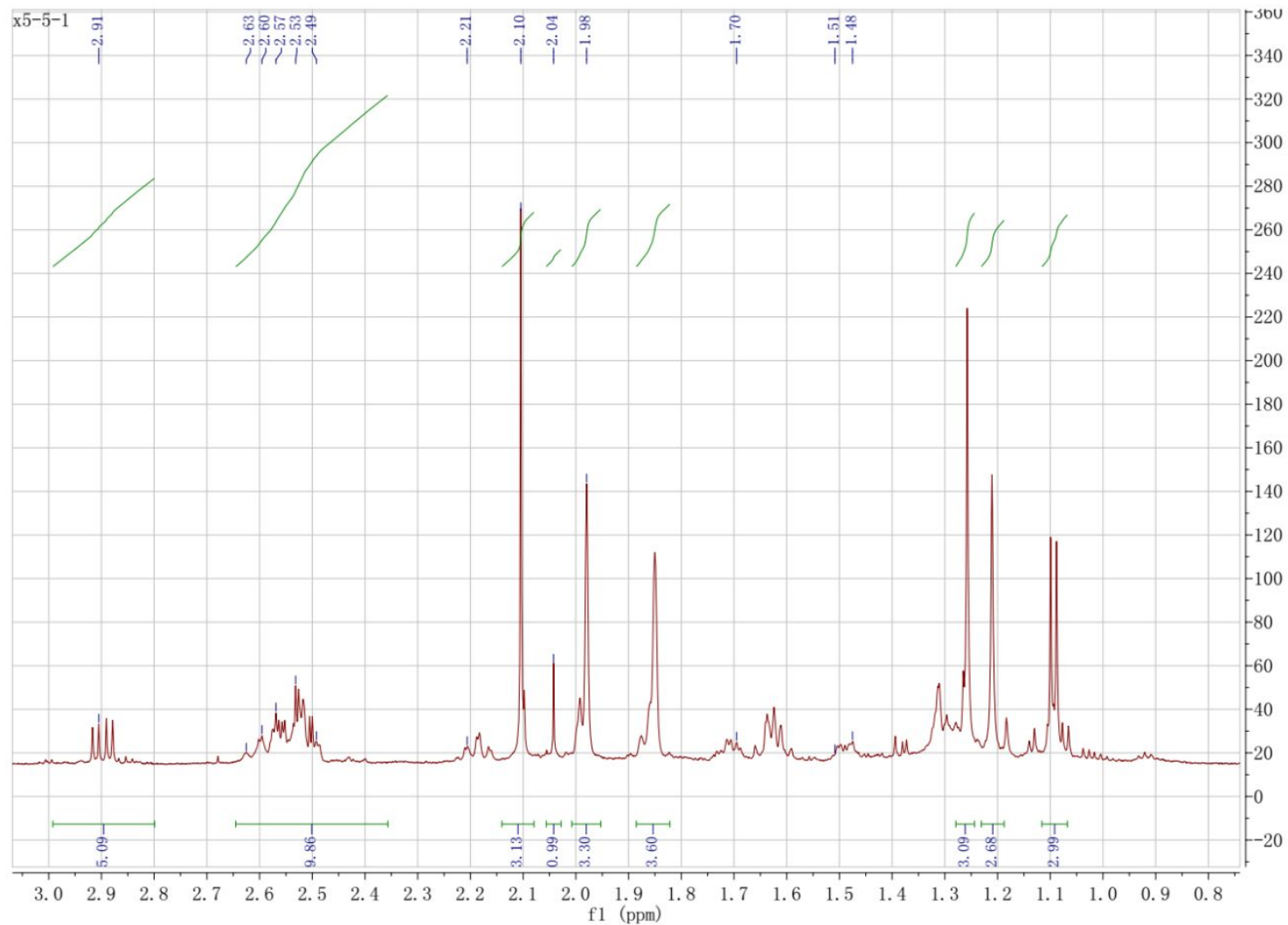


${ }^{13} \mathrm{C}$ NMR spectrum of compound 2 in $\mathrm{CD}_{3} \mathrm{OD}$ at $150 \mathrm{MHz}$

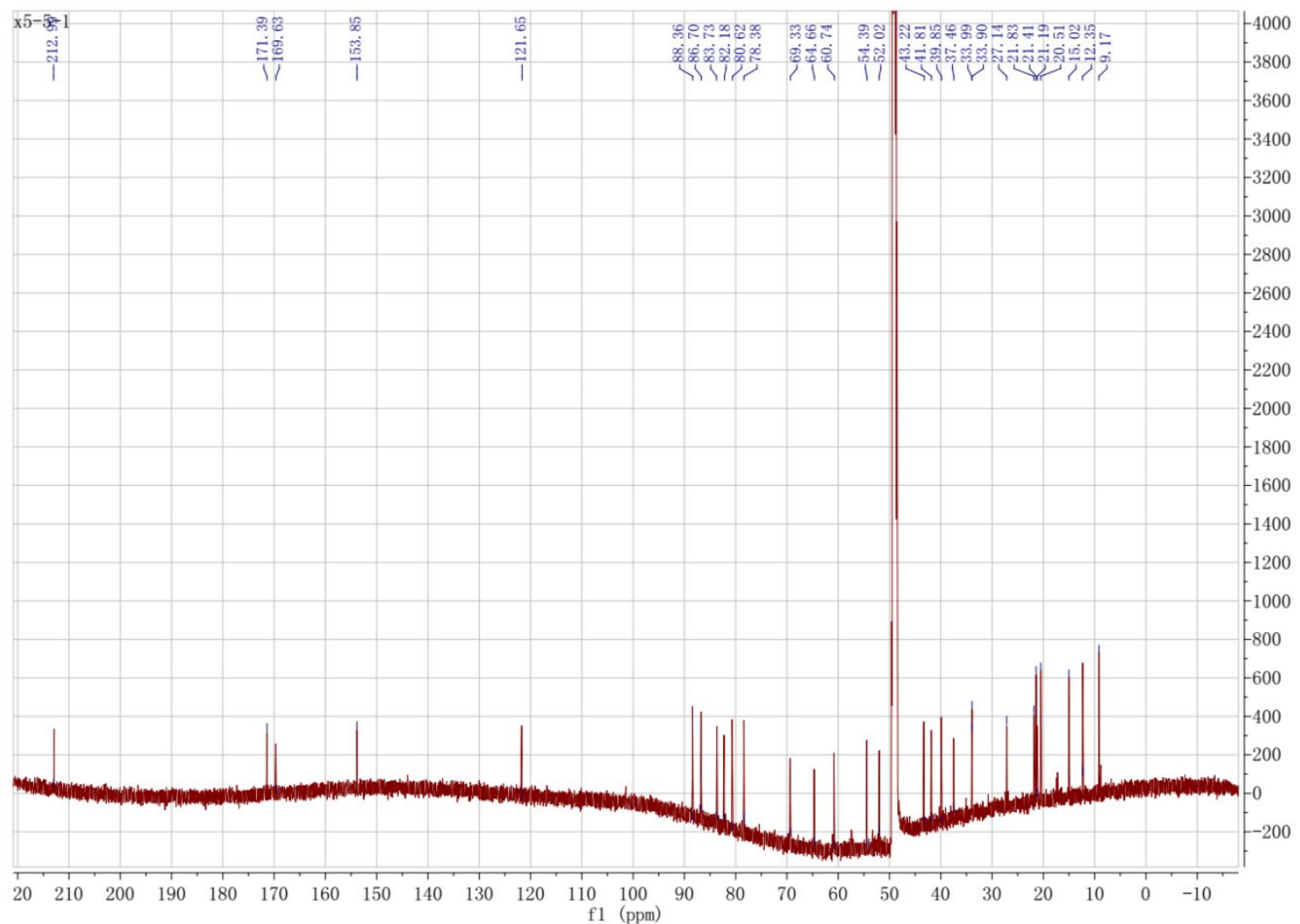

Detailed ${ }^{13} \mathrm{C}$ NMR spectrum of compound 2 in $\mathrm{CD}_{3} \mathrm{OD}$ at $150 \mathrm{MHz}$

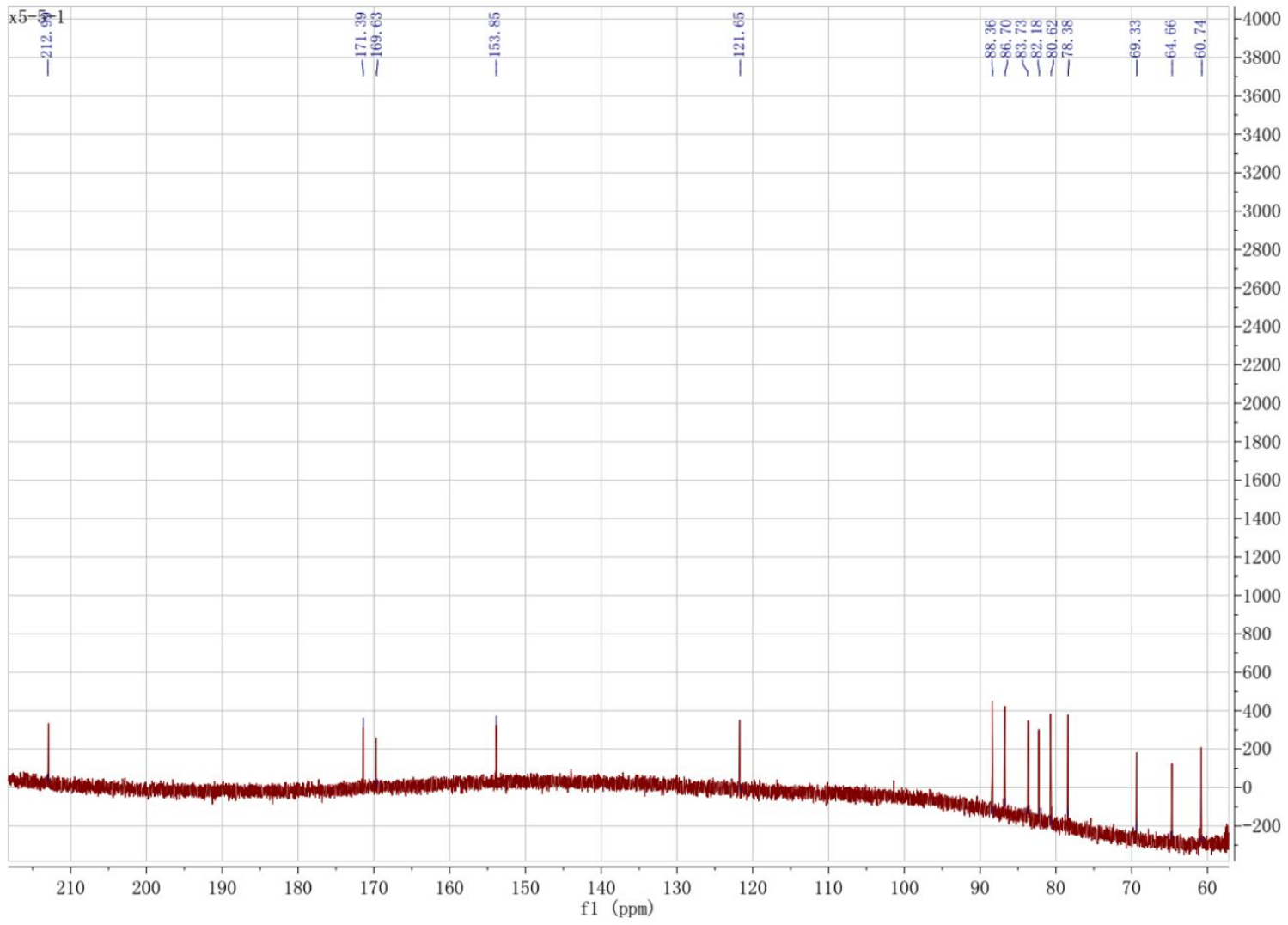


Detailed ${ }^{13} \mathrm{C}$ NMR spectrum of compound 2 in $\mathrm{CD}_{3} \mathrm{OD}$ at $150 \mathrm{MHz}$

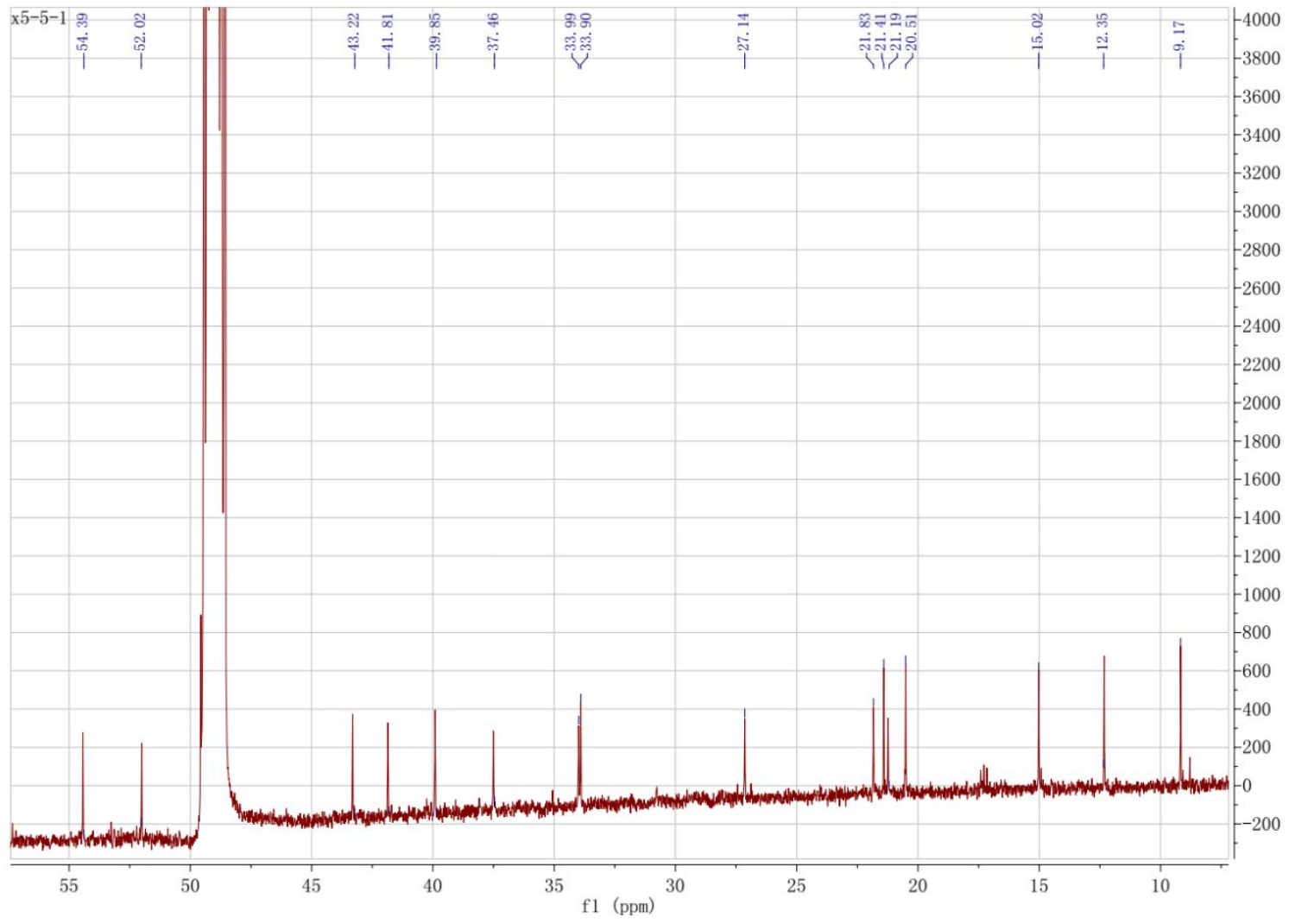

HSQC spectrum of compound 2 in $\mathrm{CD}_{3} \mathrm{OD}$

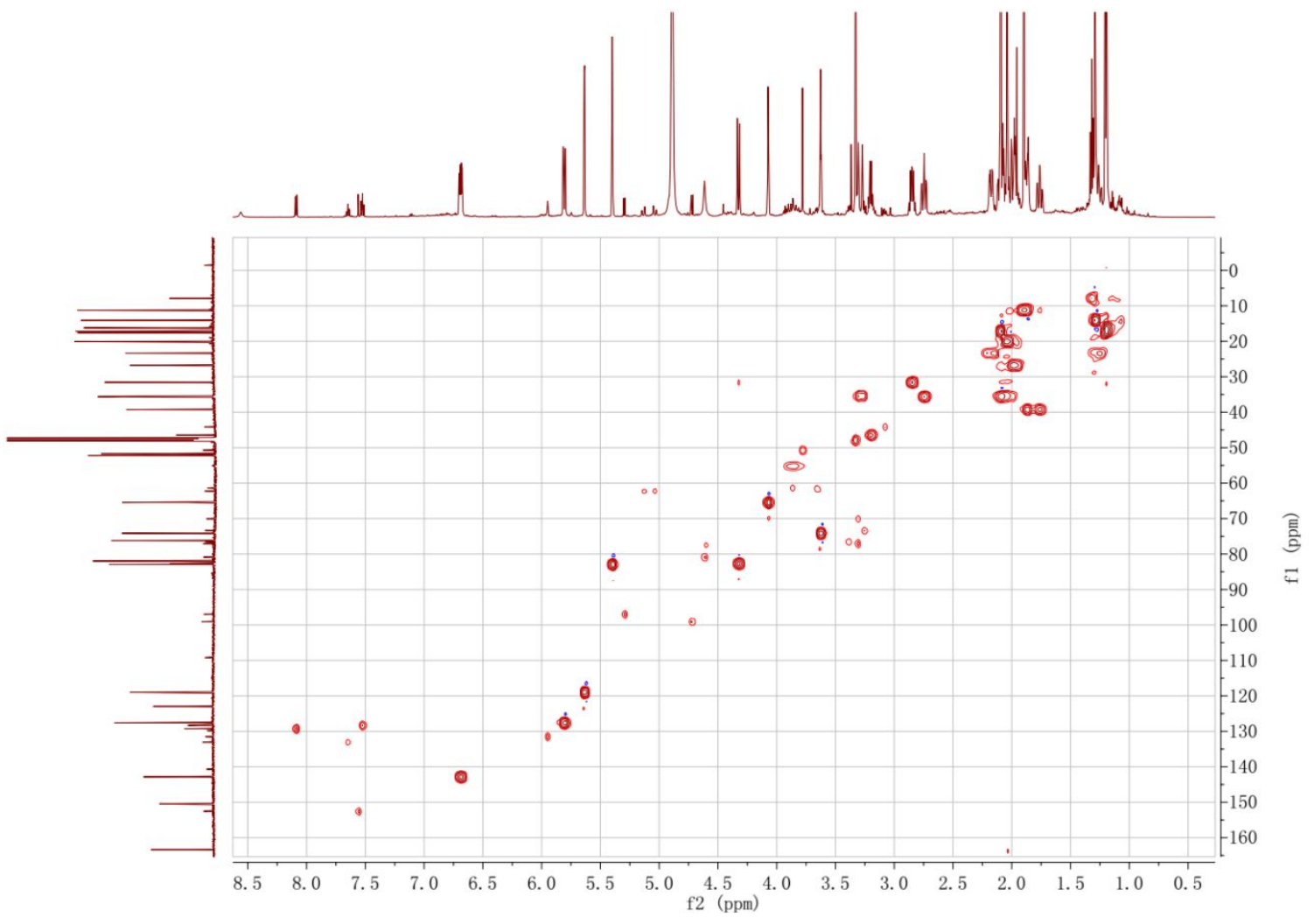


HMBC spectrum of compound 2 in $\mathrm{CD}_{3} \mathrm{OD}$

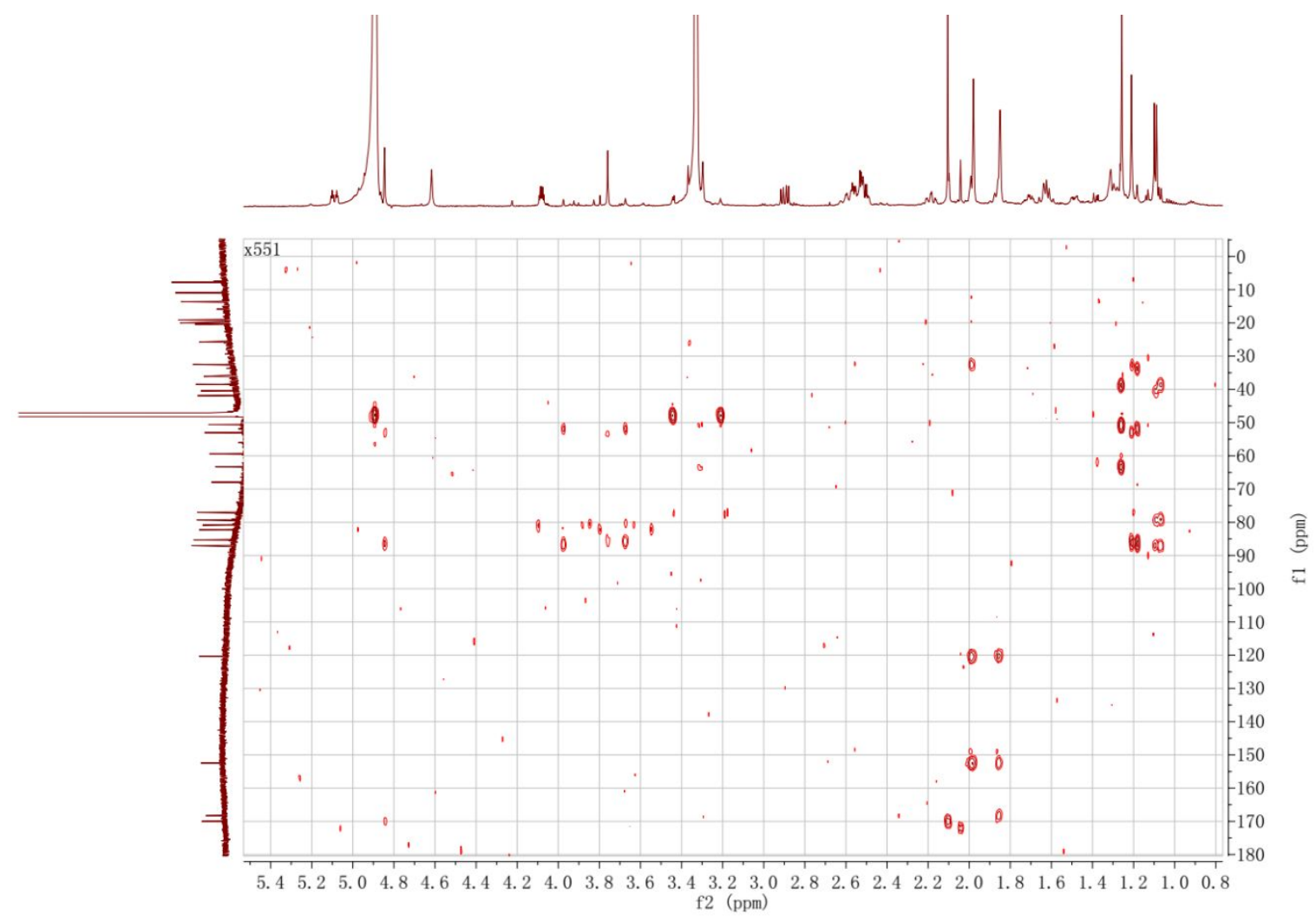

COSY spectrum of compound 2 in $\mathrm{CD}_{3} \mathrm{OD}$

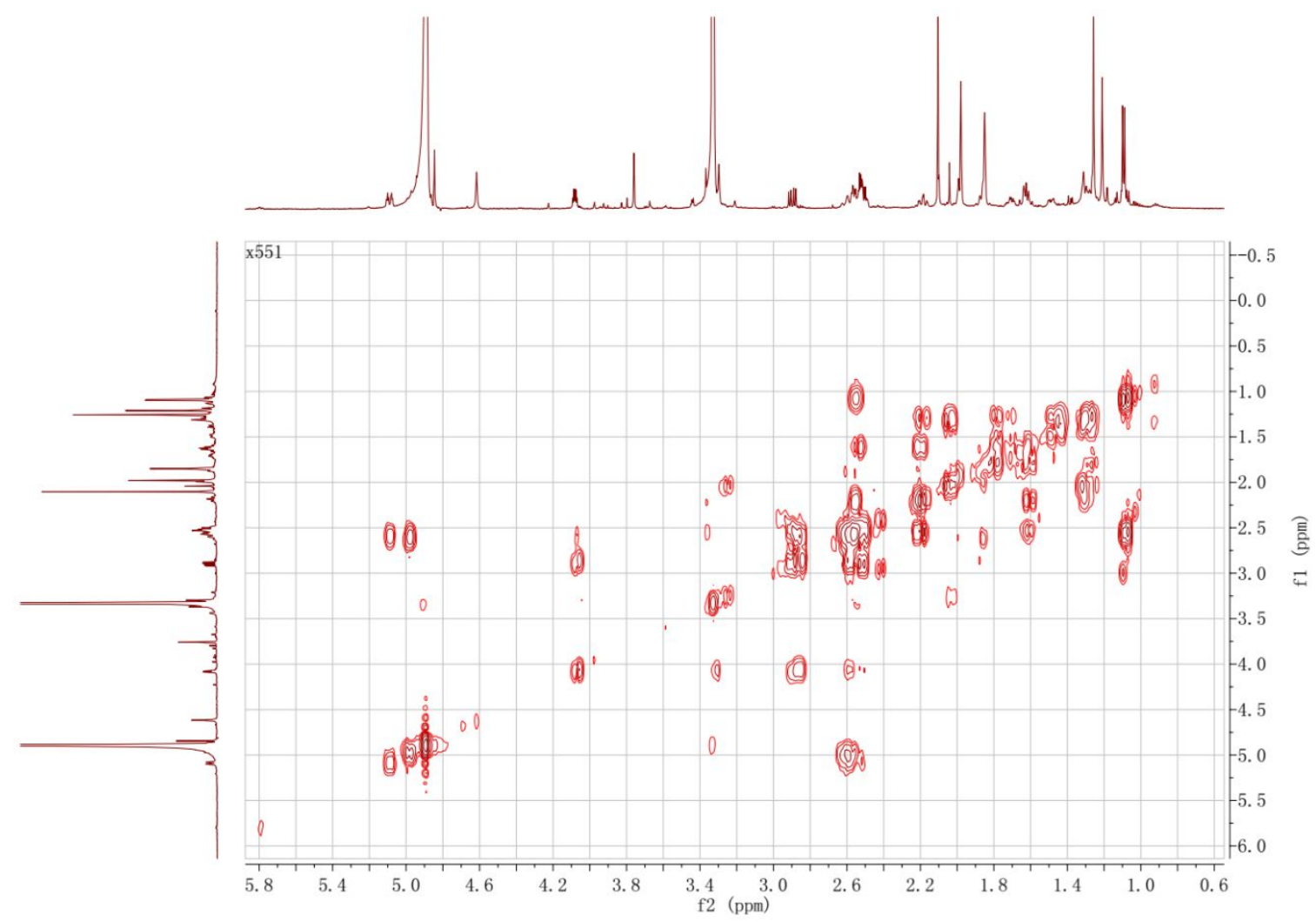


NOESY spectrum of compound 2 in $\mathrm{CD}_{3} \mathrm{OD}$

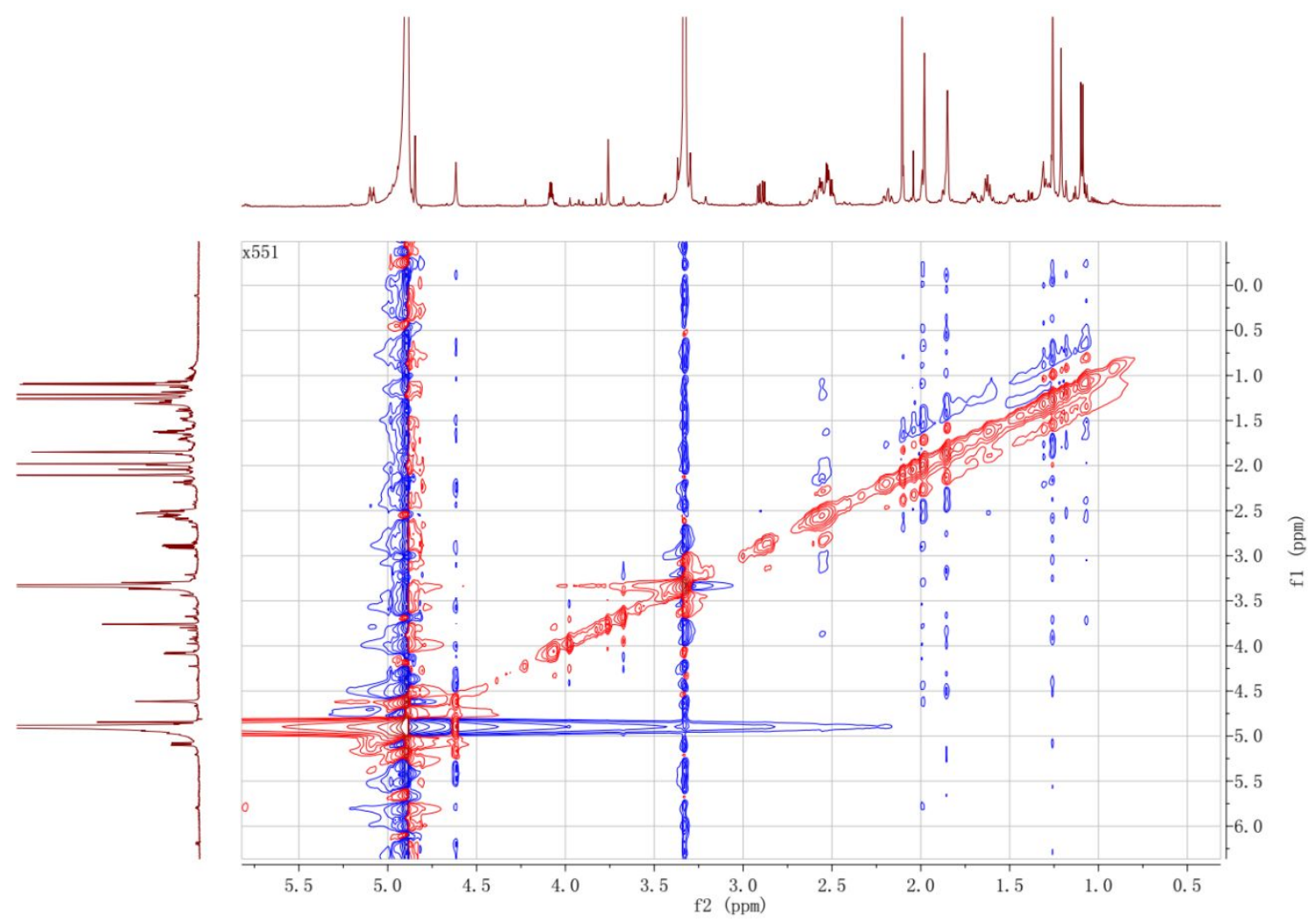

S18 
HR-ESI-MS spectrum of compound $\mathbf{3}$

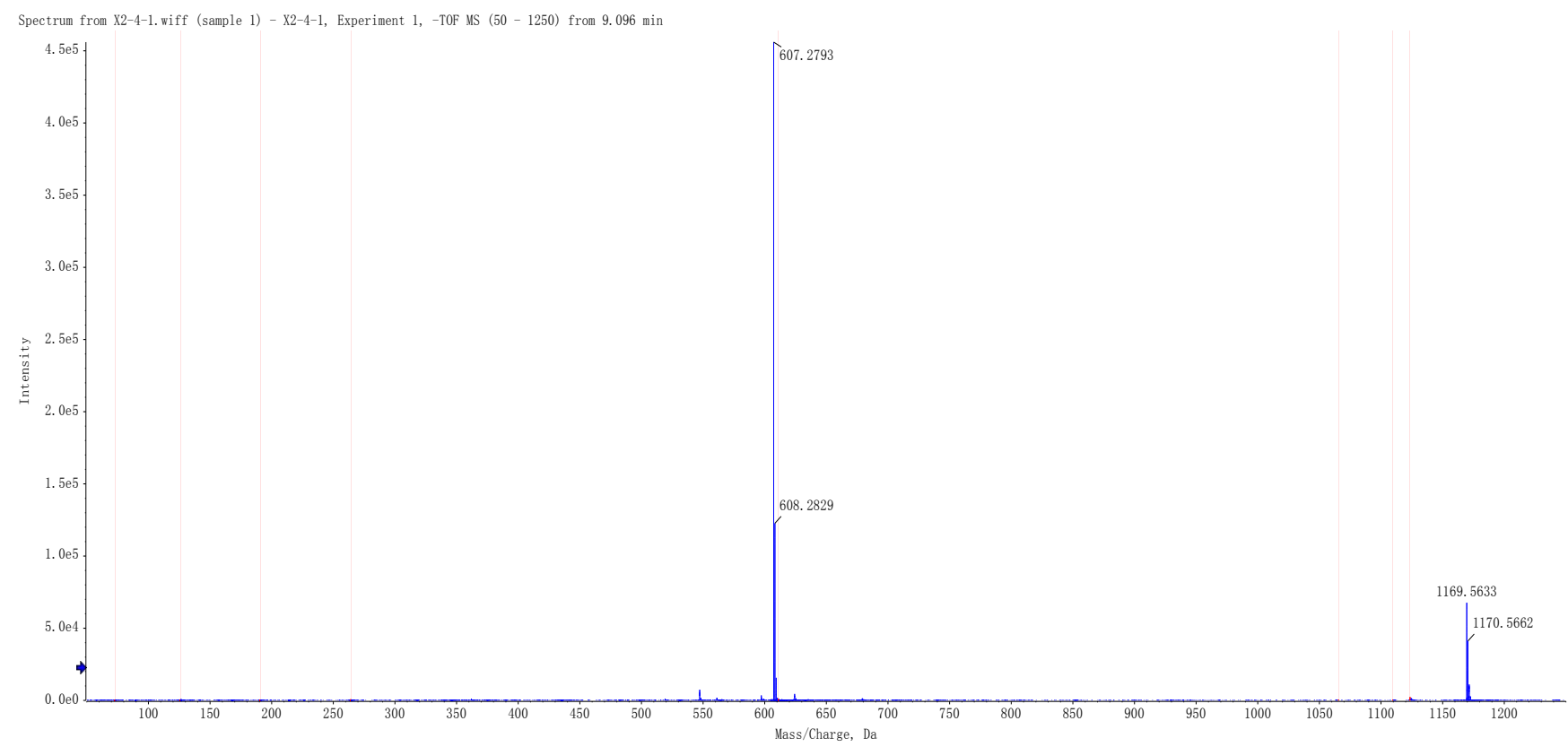

${ }^{1} \mathrm{H}$ NMR spectrum of compound 3 in $\mathrm{CD}_{3} \mathrm{OD}$ at $600 \mathrm{MHz}$

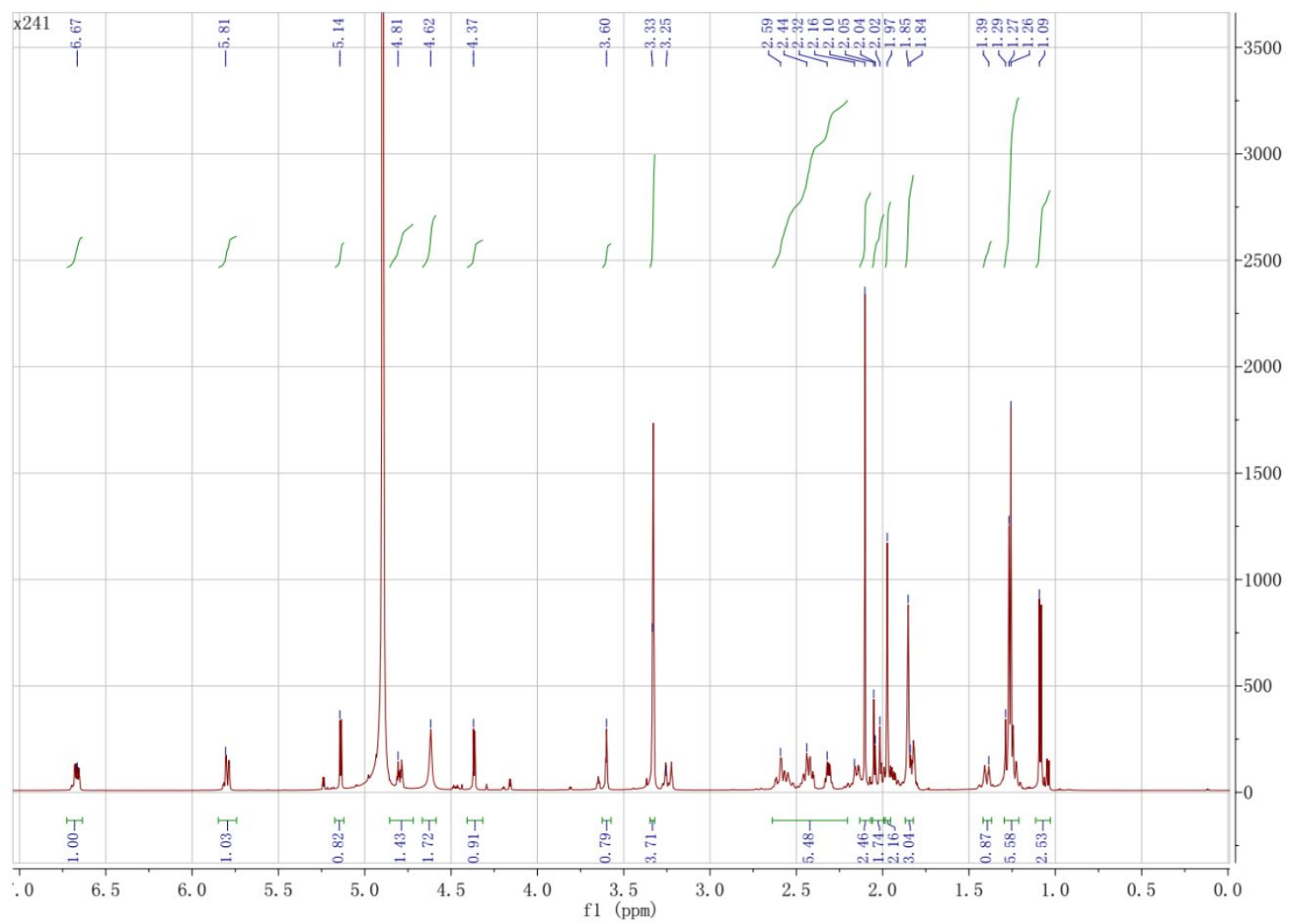


Detailed ${ }^{1} \mathrm{H}$ NMR spectrum of compound 3 in $\mathrm{CD}_{3} \mathrm{OD}$ at $600 \mathrm{MHz}$

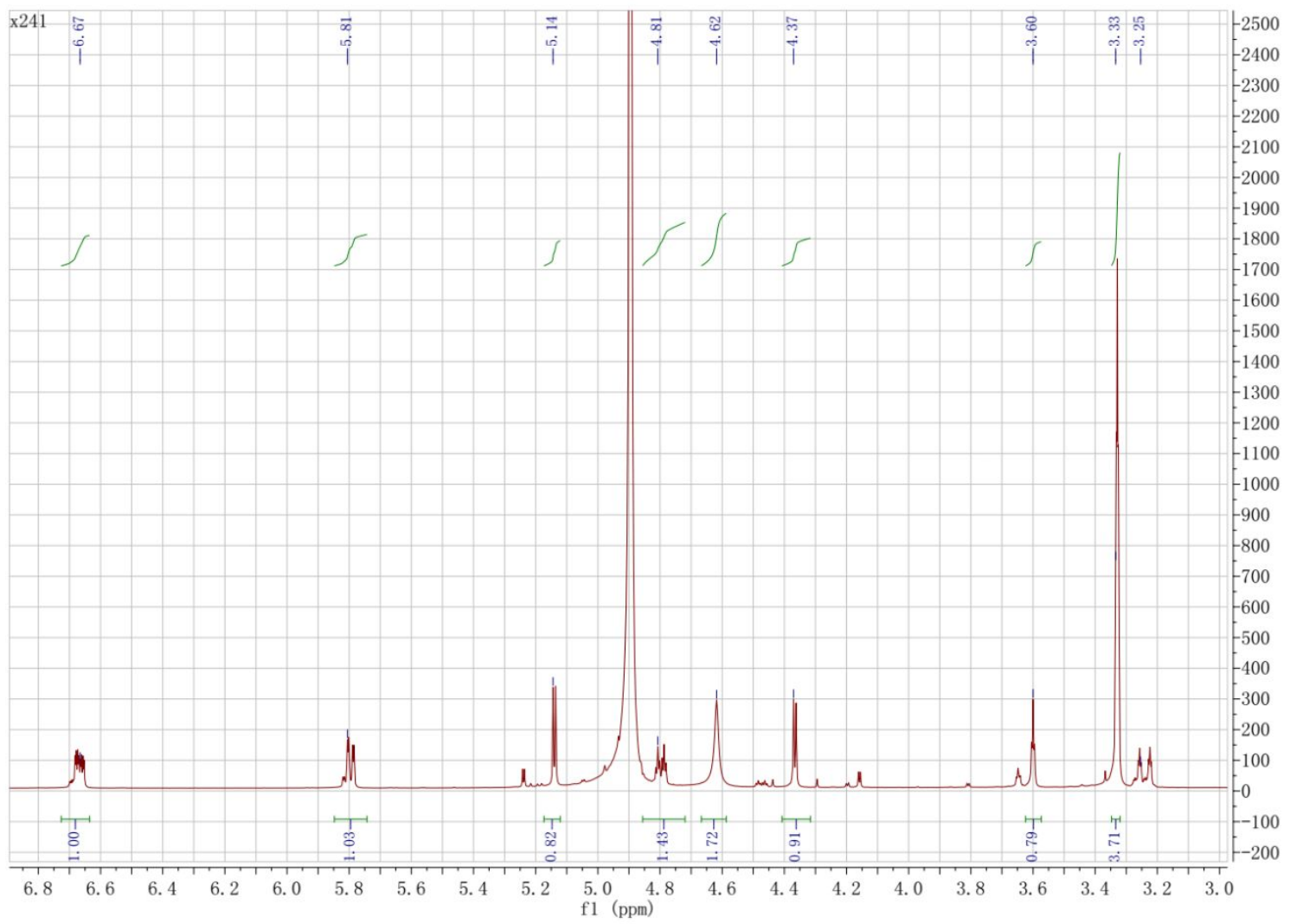

Detailed ${ }^{1} \mathrm{H}$ NMR spectrum of compound 3 in $\mathrm{CD}_{3} \mathrm{OD}$ at $600 \mathrm{MHz}$

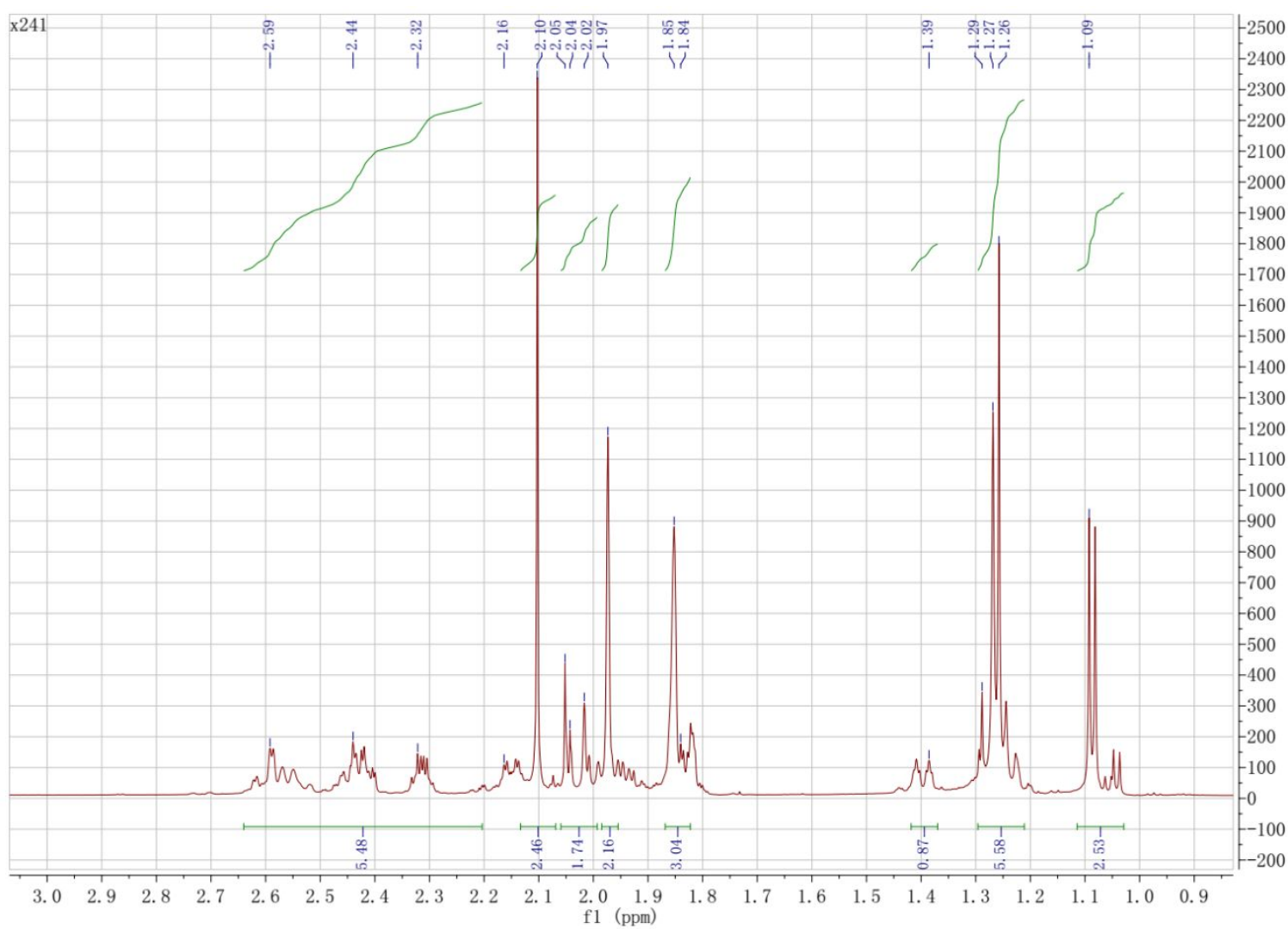


${ }^{13} \mathrm{C}$ NMR spectrum of compound 3 in $\mathrm{CD}_{3} \mathrm{OD}$ at $150 \mathrm{MHz}$

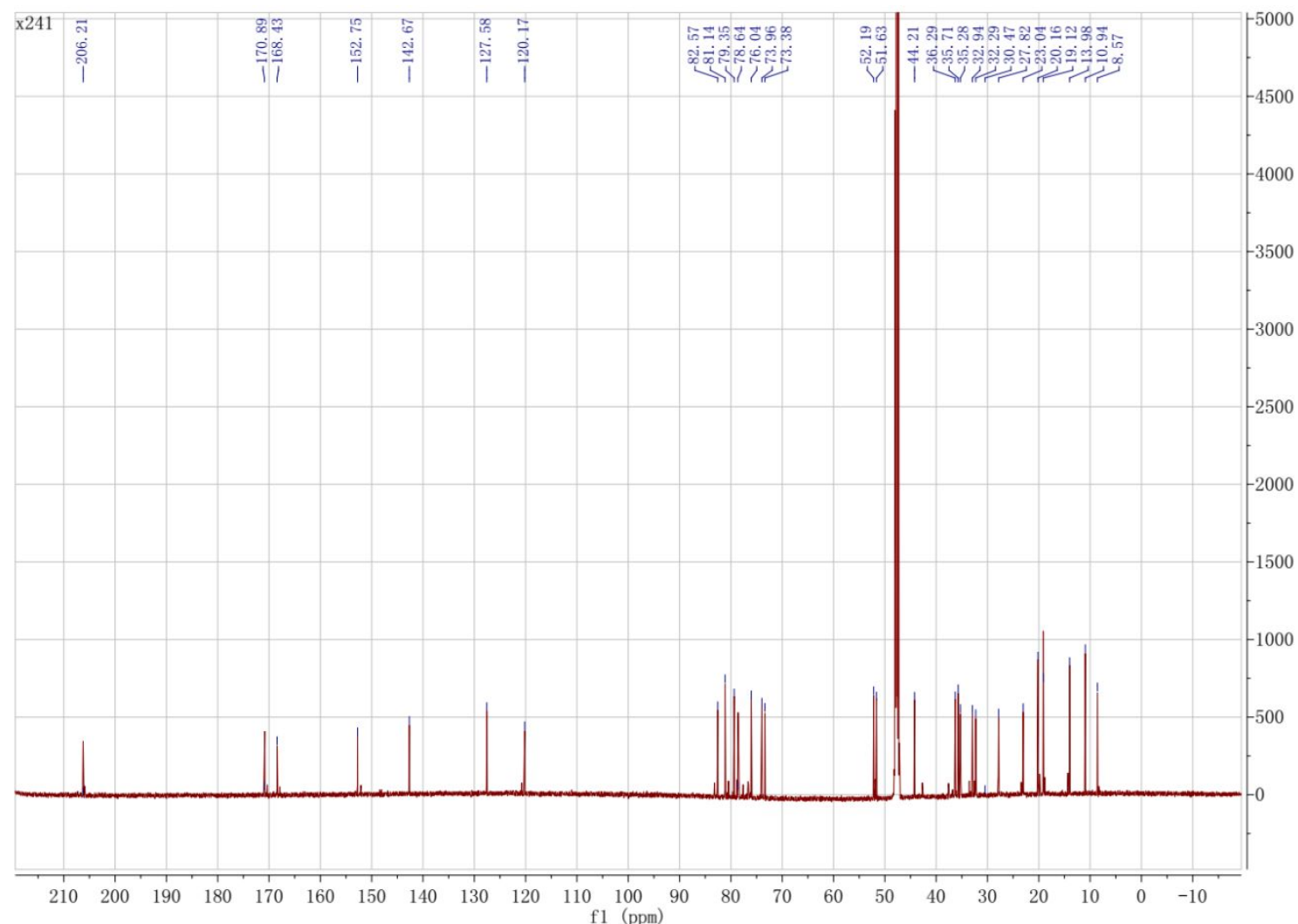

Detailed ${ }^{13} \mathrm{C}$ NMR spectrum of compound 3 in $\mathrm{CD}_{3} \mathrm{OD}$ at $150 \mathrm{MHz}$

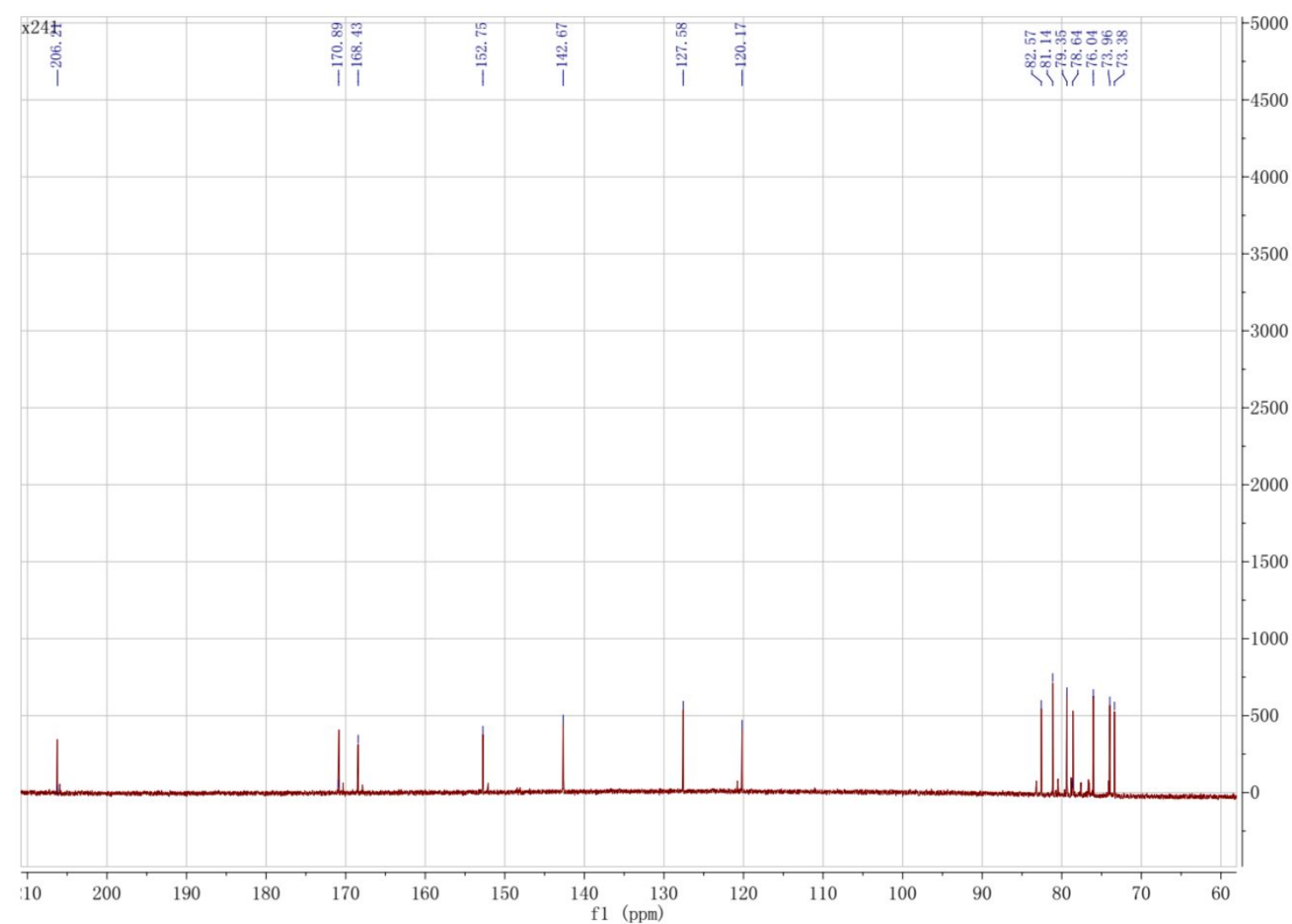


Detailed ${ }^{13} \mathrm{C}$ NMR spectrum of compound 3 in $\mathrm{CD}_{3} \mathrm{OD}$ at $150 \mathrm{MHz}$

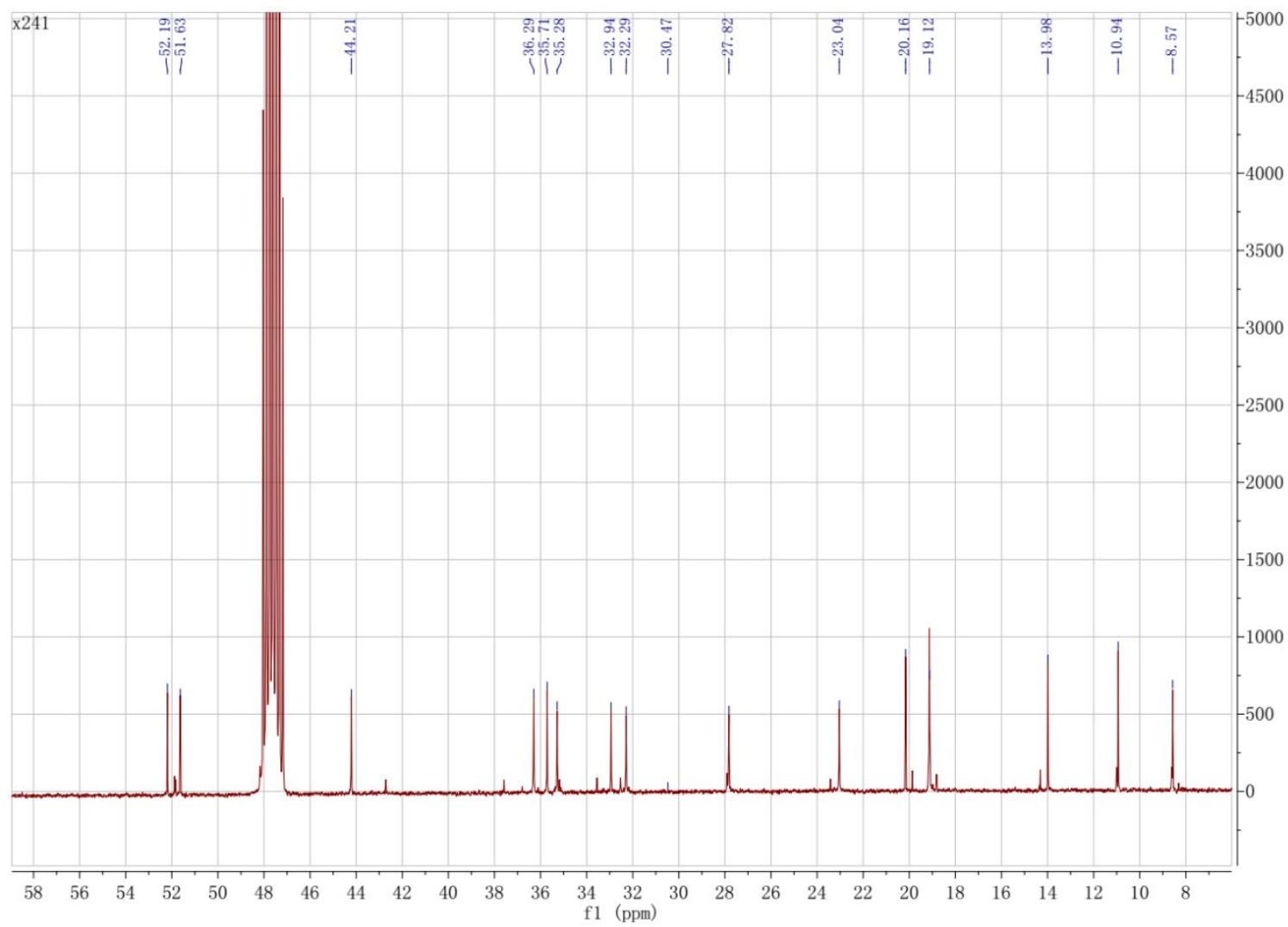

HSQC spectrum of compound 3 in $\mathrm{CD}_{3} \mathrm{OD}$

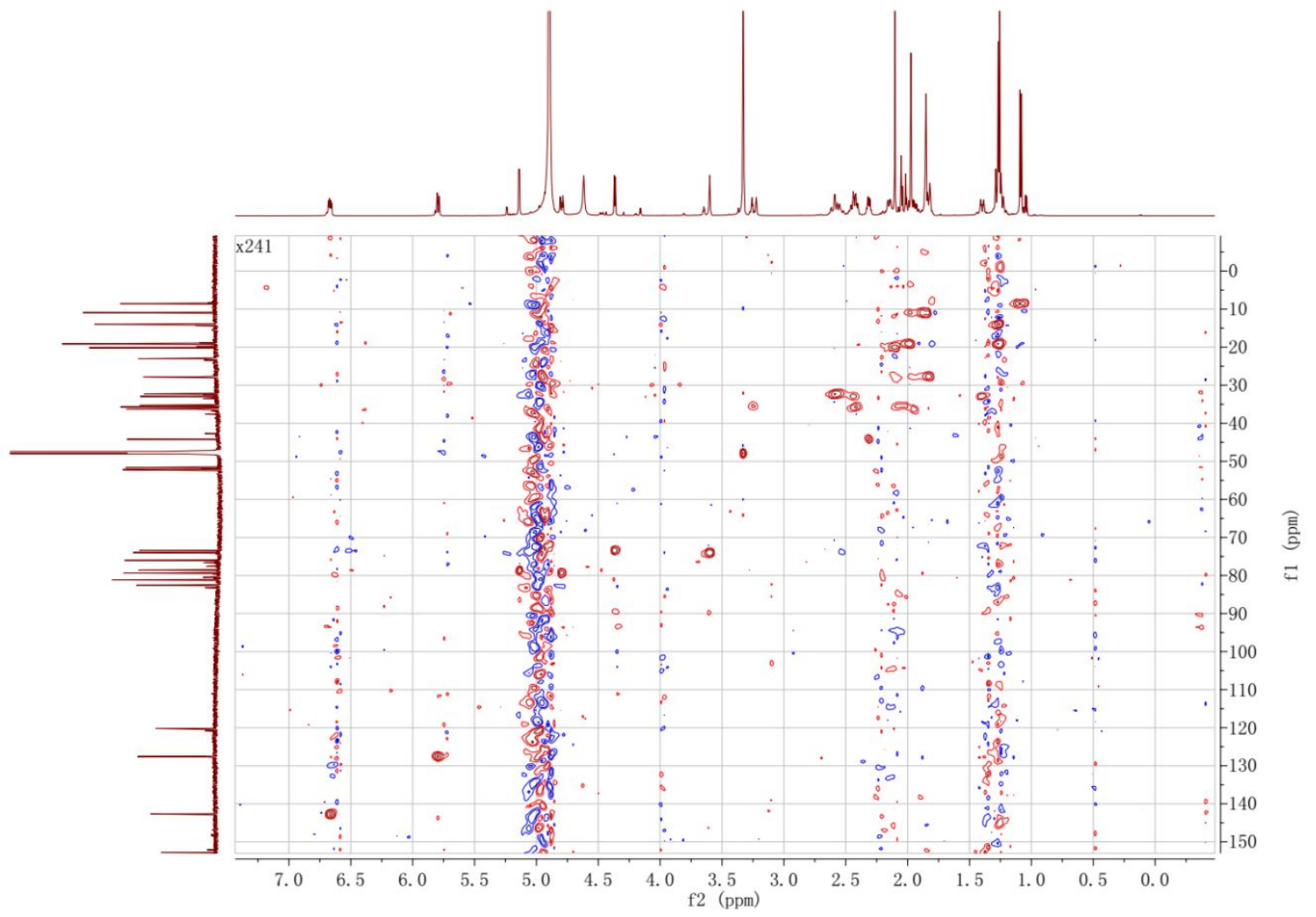


$\mathrm{HMBC}$ spectrum of compound 3 in $\mathrm{CD}_{3} \mathrm{OD}$

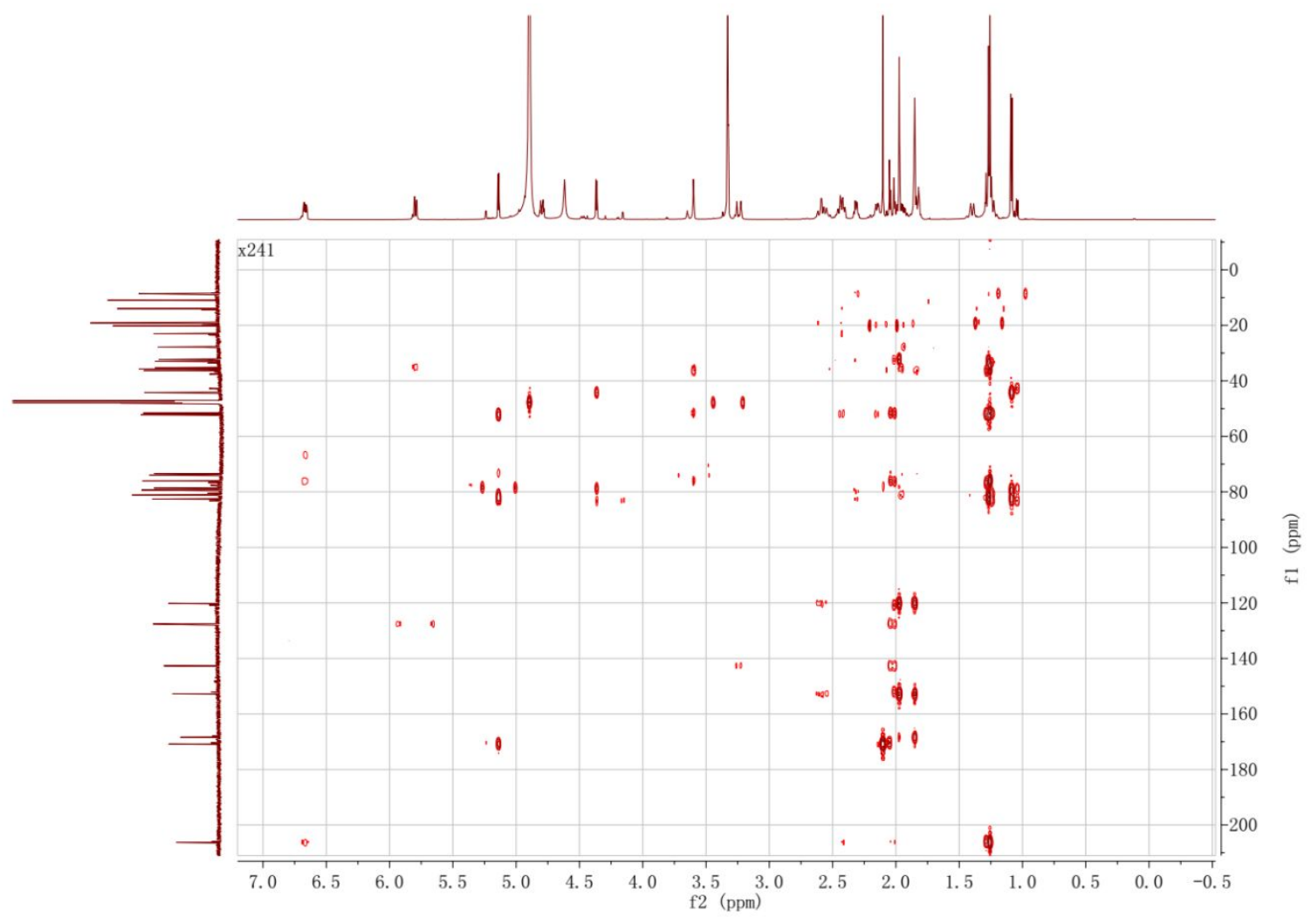

COSY spectrum of compound 3 in $\mathrm{CD}_{3} \mathrm{OD}$

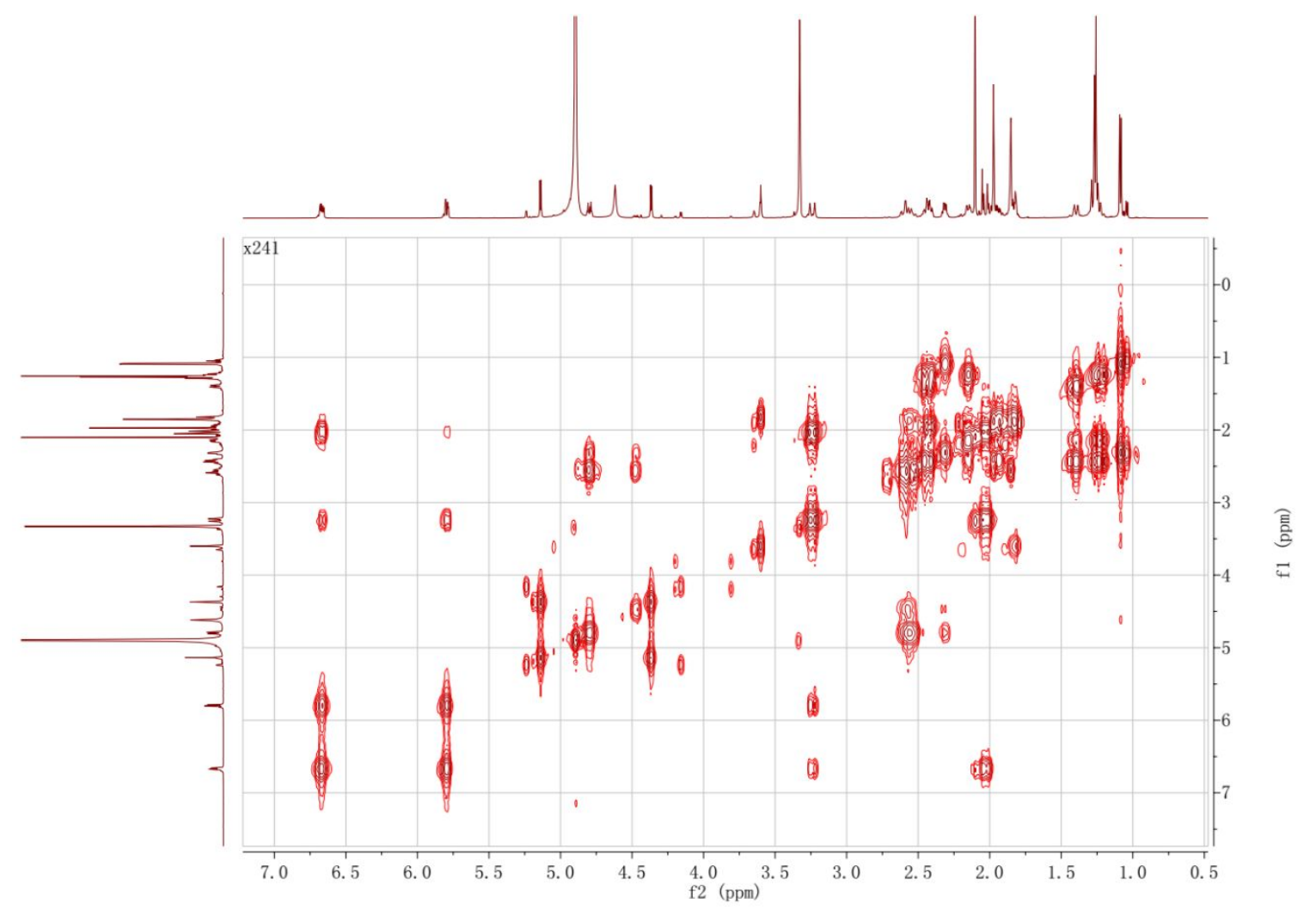


NOESY spectrum of compound $\mathbf{3}$ in $\mathrm{CD}_{3} \mathrm{OD}$

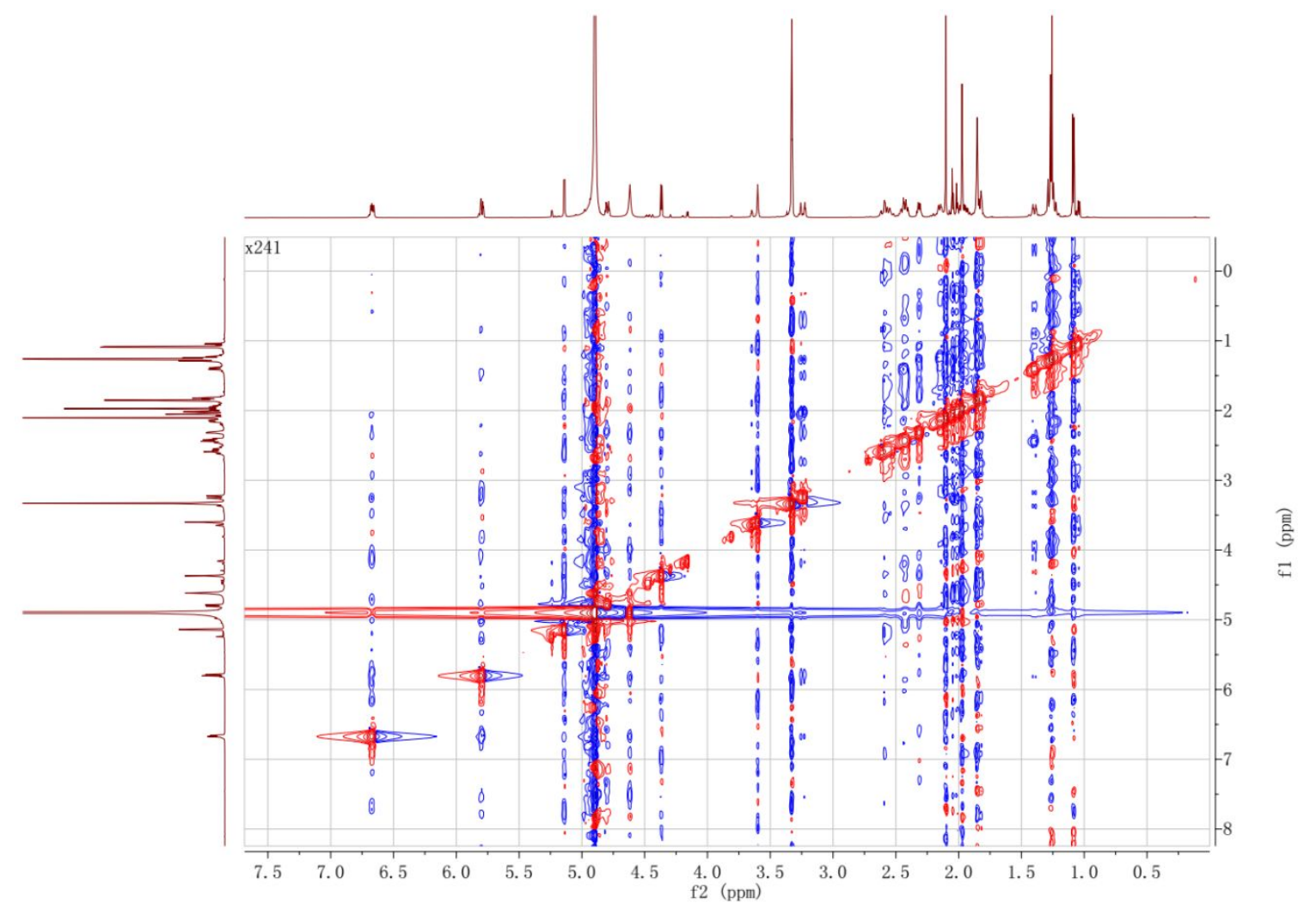


HR-ESI-MS spectrum of compound 4

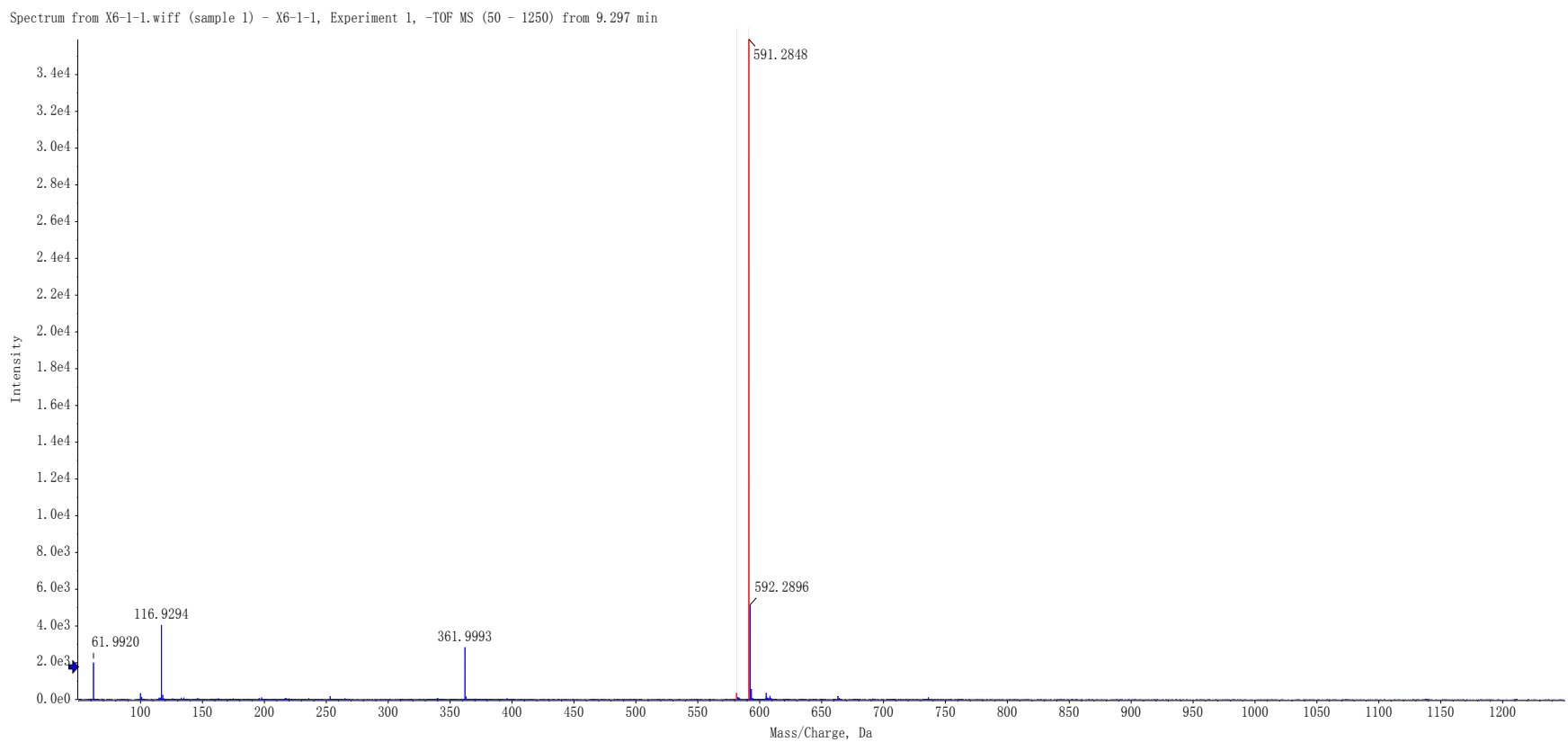

${ }^{1} \mathrm{H}$ NMR spectrum of compound 4 in $\mathrm{CD}_{3} \mathrm{OD}$ at $600 \mathrm{MHz}$

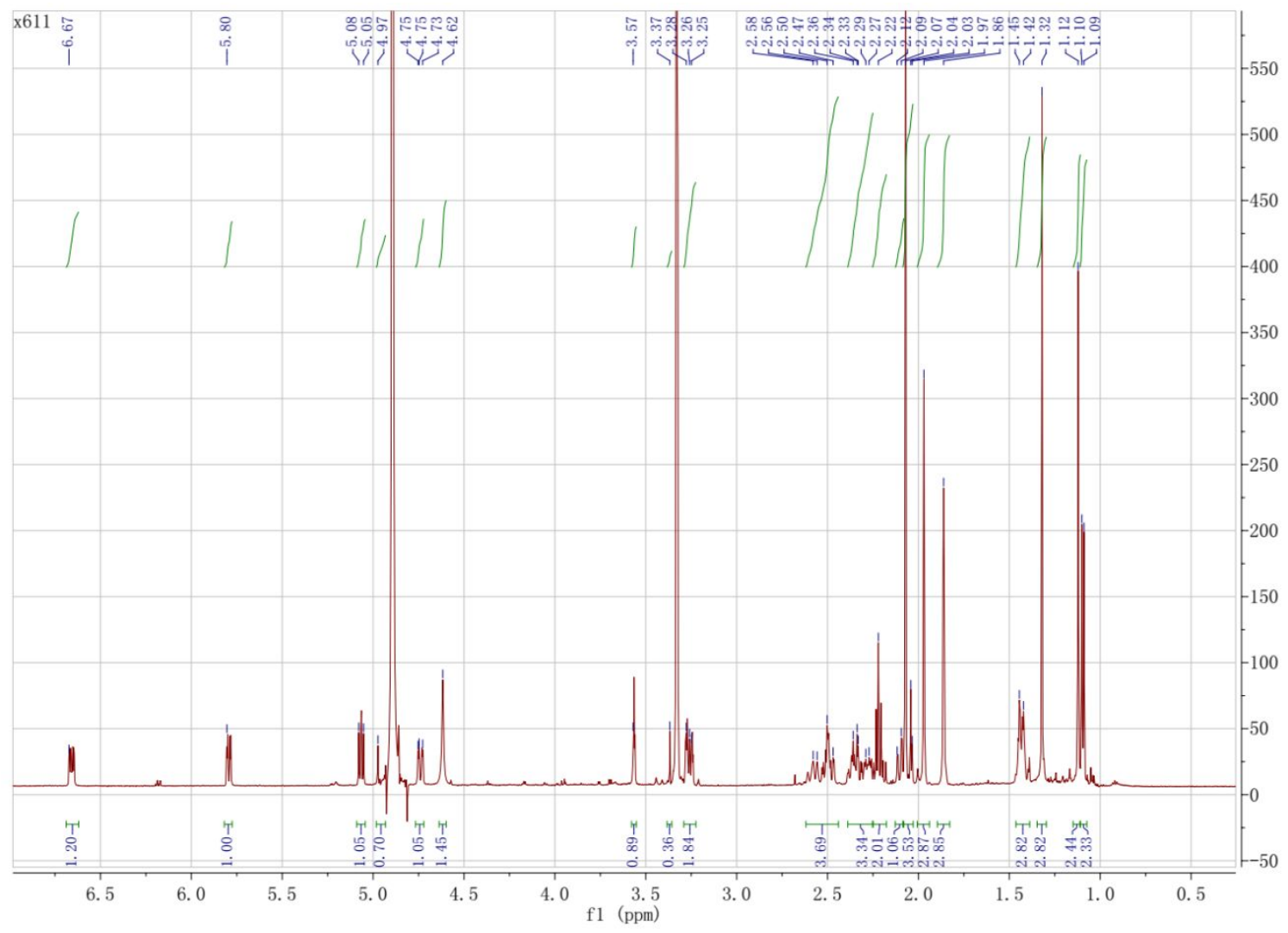


Detailed ${ }^{1} \mathrm{H}$ NMR spectrum of compound 4 in $\mathrm{CD}_{3} \mathrm{OD}$ at $600 \mathrm{MHz}$

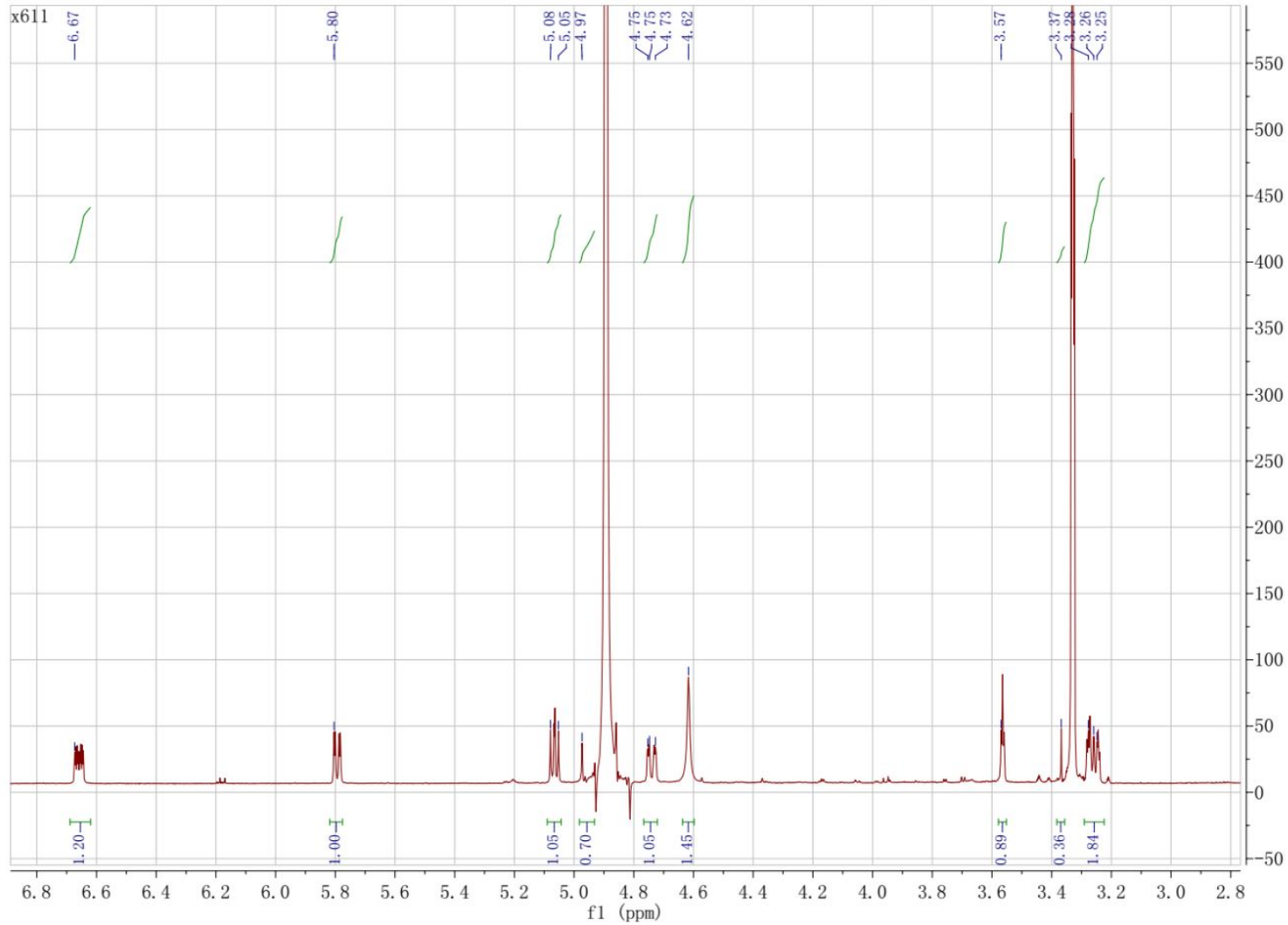

Detailed ${ }^{1} \mathrm{H}$ NMR spectrum of compound 4 in $\mathrm{CD}_{3} \mathrm{OD}$ at $600 \mathrm{MHz}$

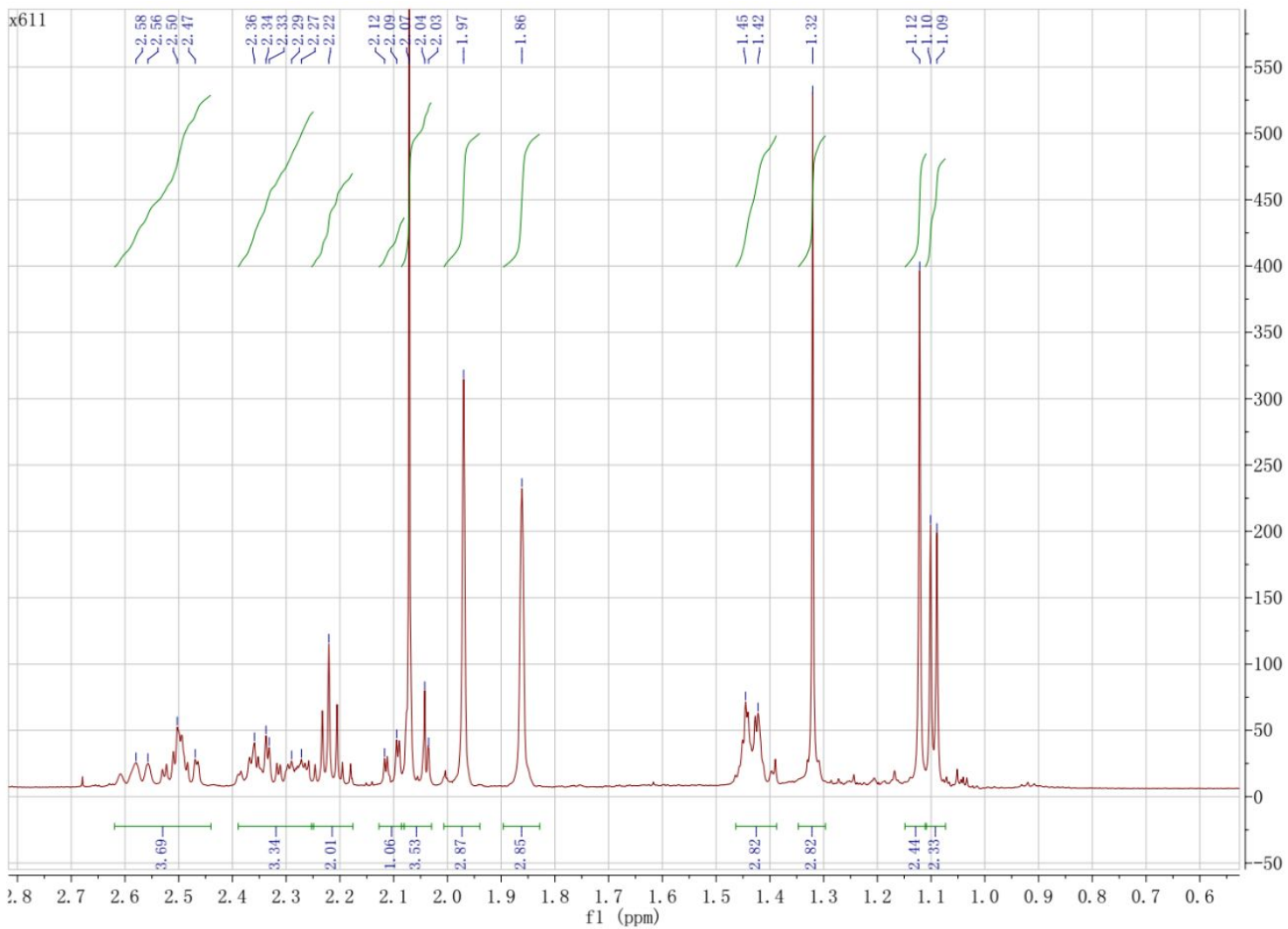


${ }^{13} \mathrm{C}$ NMR spectrum of compound 4 in $\mathrm{CD}_{3} \mathrm{OD}$ at $150 \mathrm{MHz}$

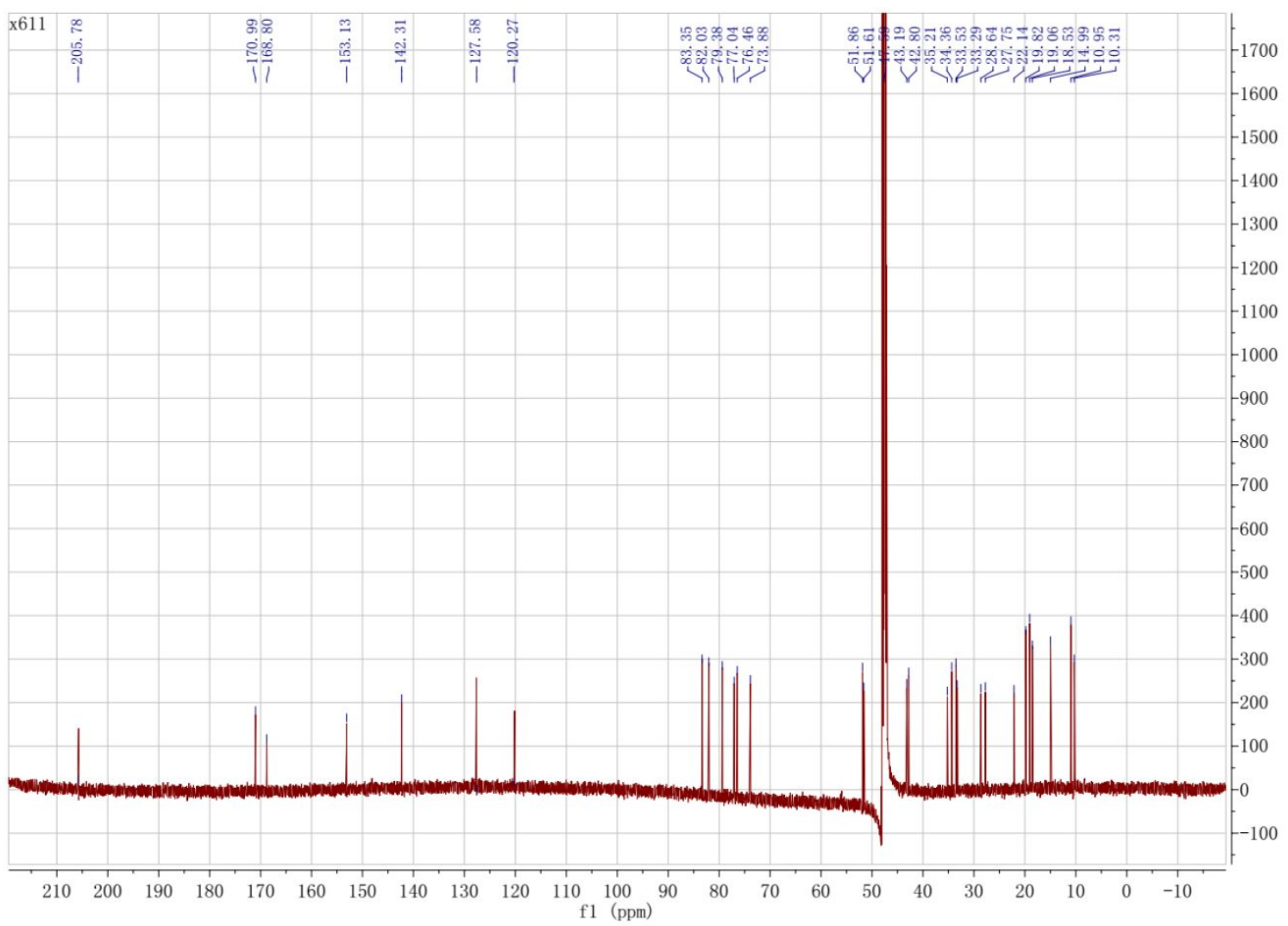

Detailed ${ }^{13} \mathrm{C}$ NMR spectrum of compound 4 in $\mathrm{CD}_{3} \mathrm{OD}$ at $150 \mathrm{MHz}$

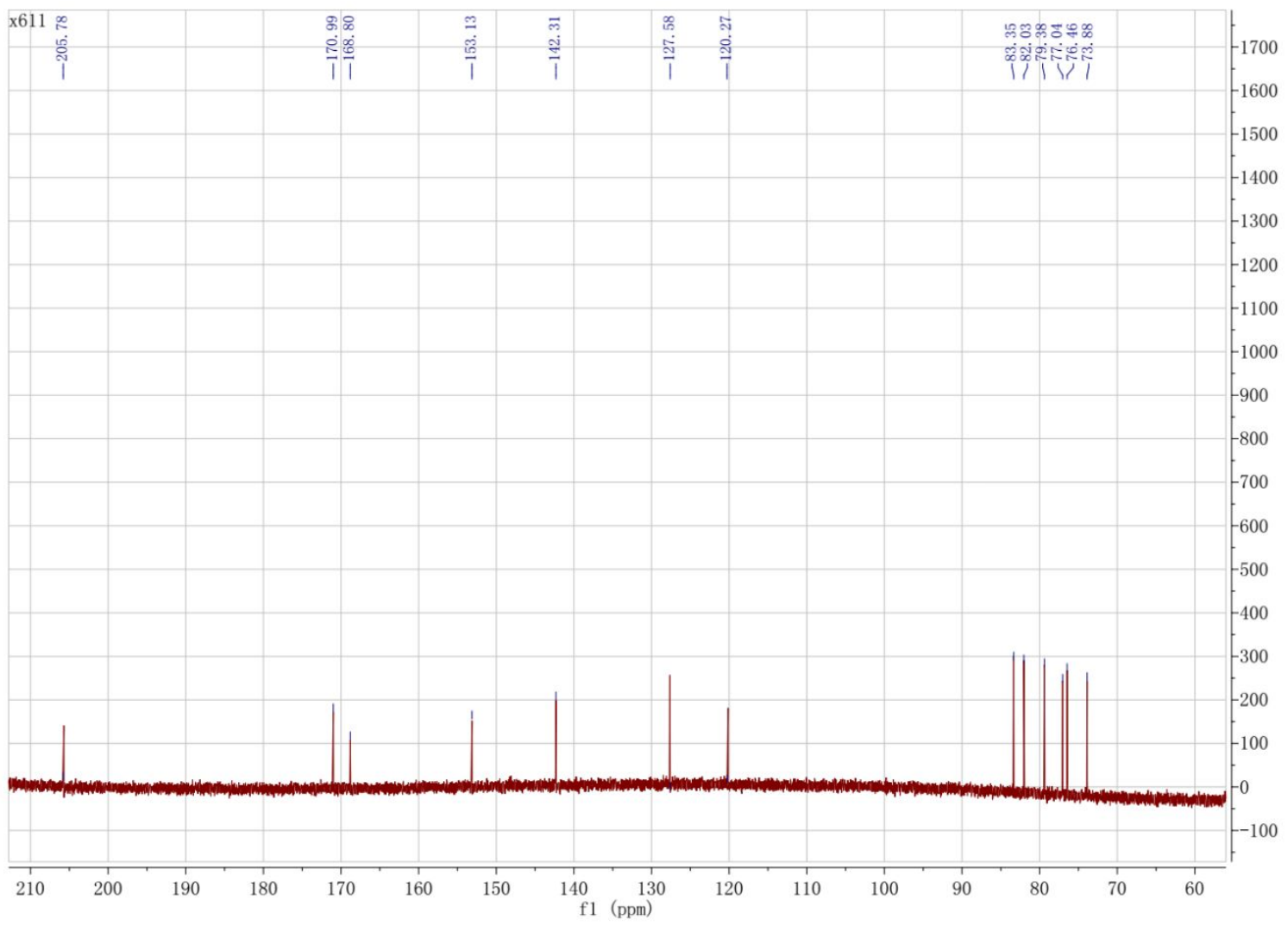


Detailed ${ }^{13} \mathrm{C}$ NMR spectrum of compound 4 in $\mathrm{CD}_{3} \mathrm{OD}$ at $150 \mathrm{MHz}$

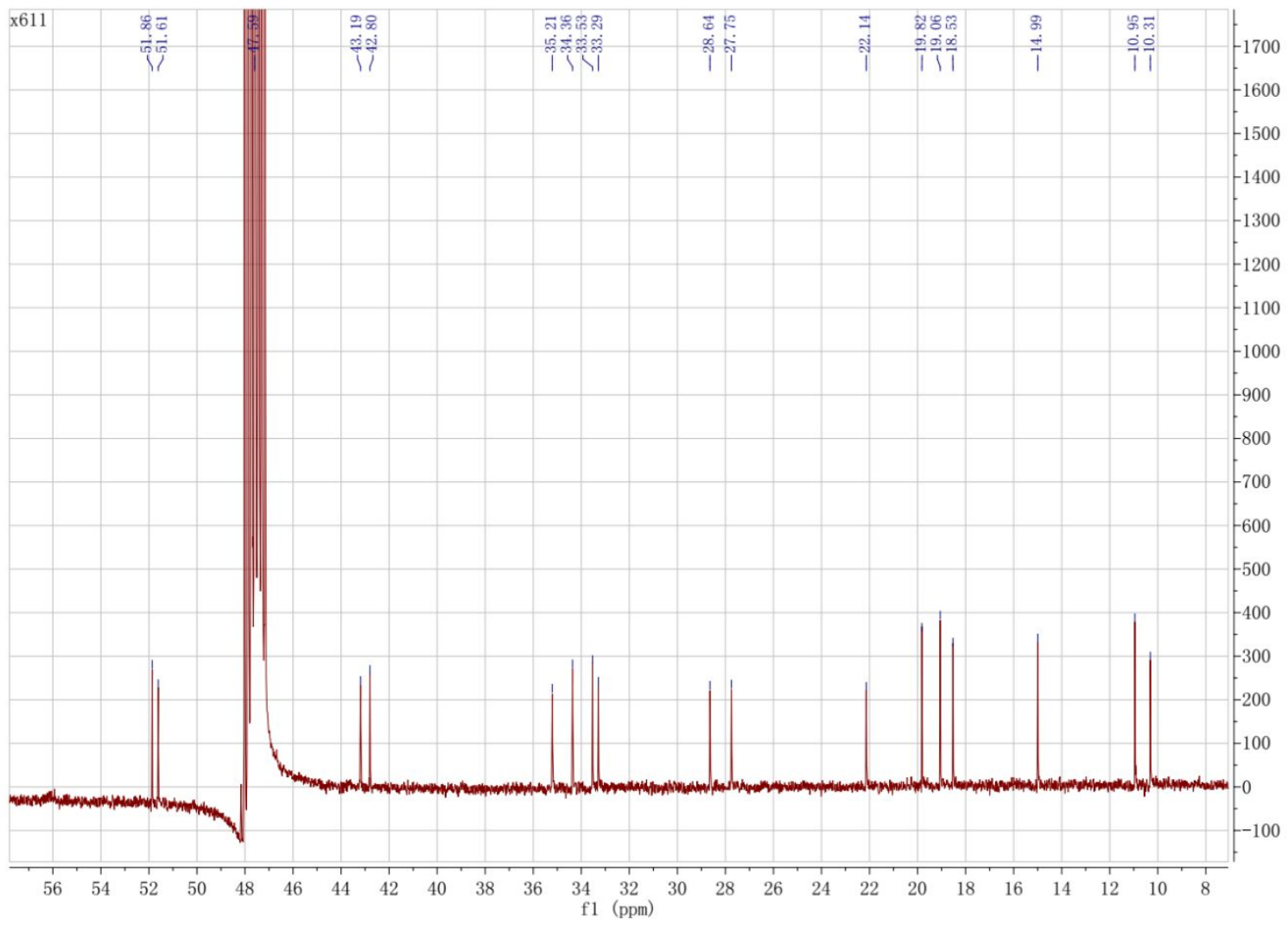

HSQC spectrum of compound 4 in $\mathrm{CD}_{3} \mathrm{OD}$

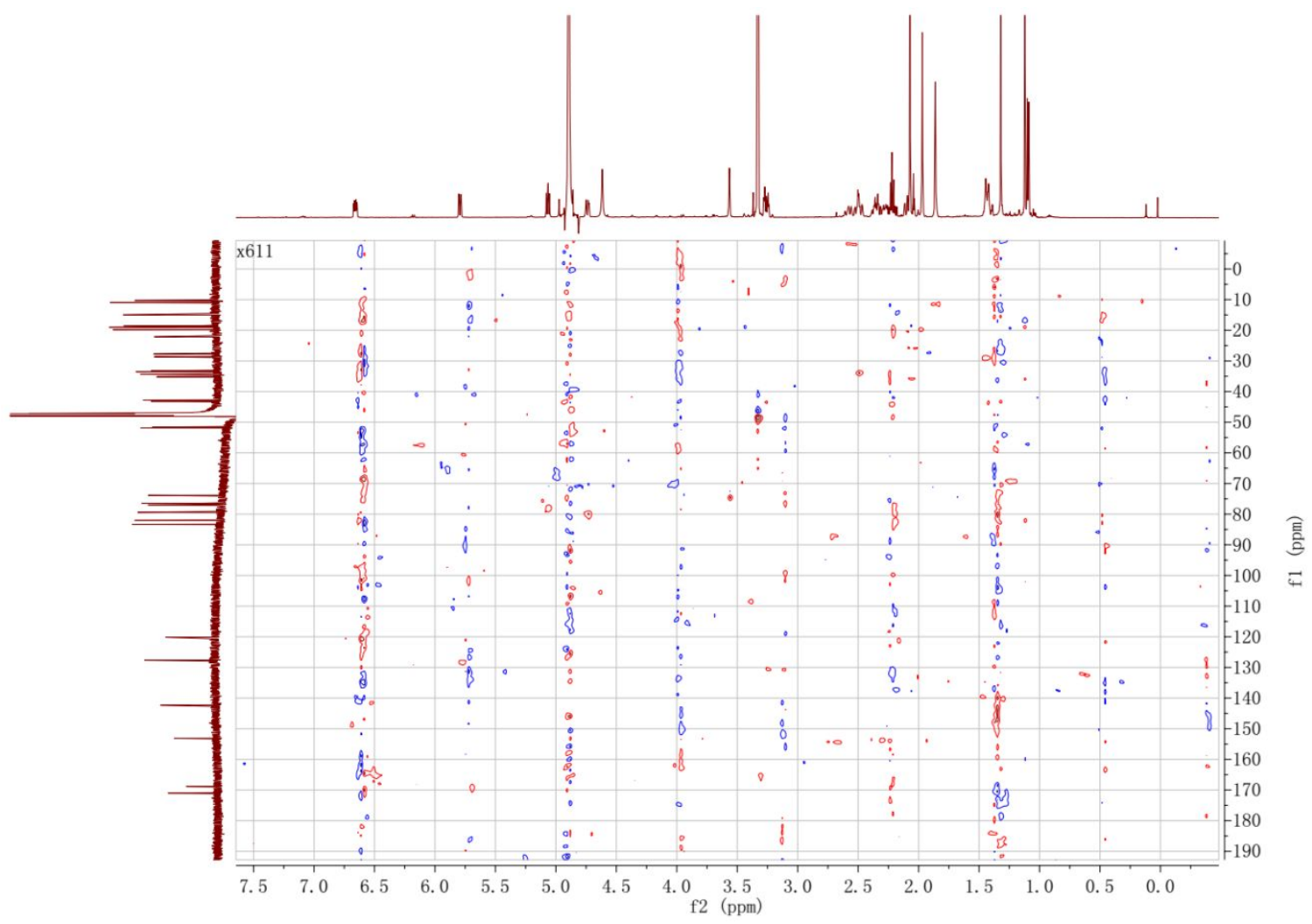


$\mathrm{HMBC}$ spectrum of compound 4 in $\mathrm{CD}_{3} \mathrm{OD}$

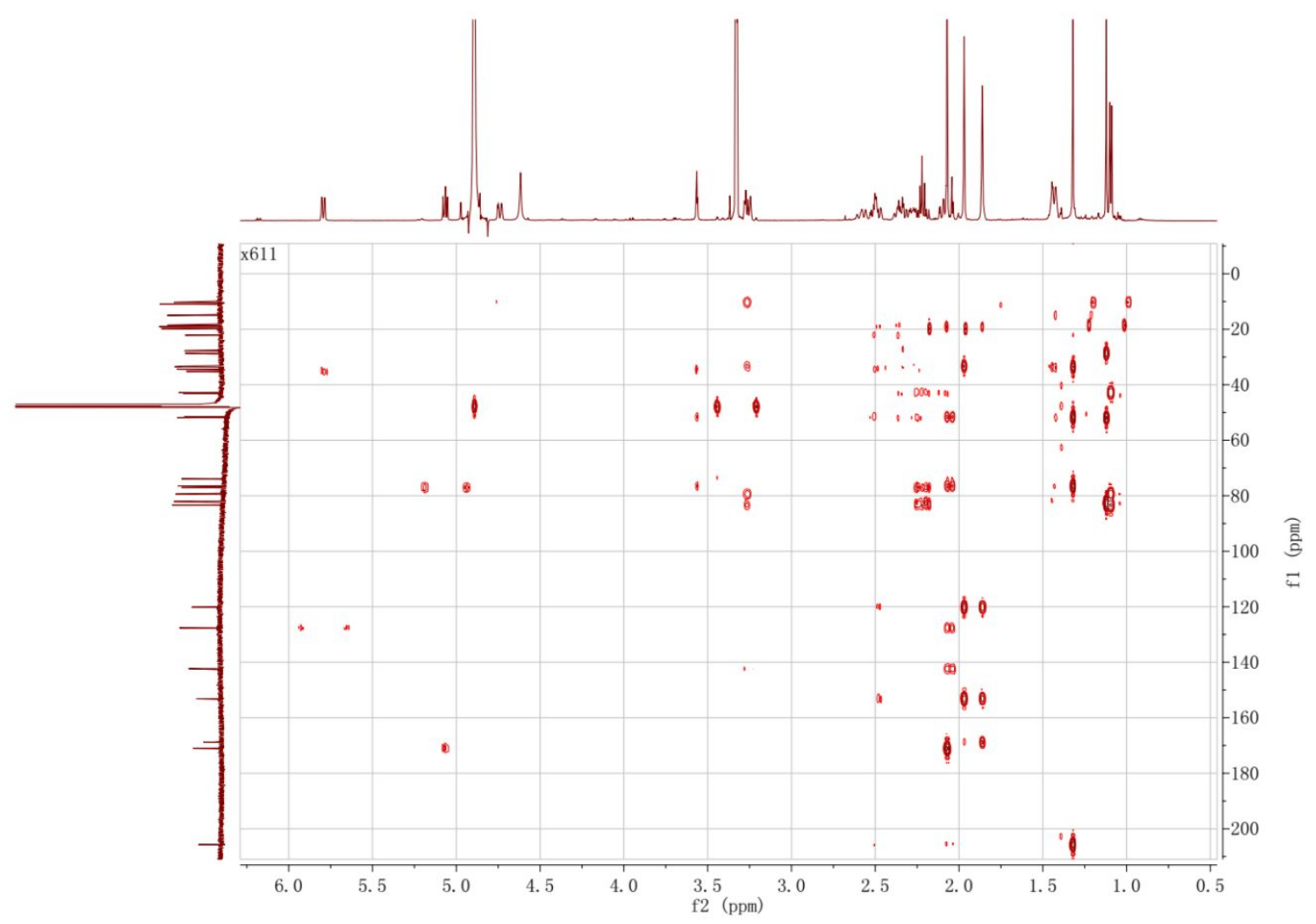

COSY spectrum of compound 4 in $\mathrm{CD}_{3} \mathrm{OD}$

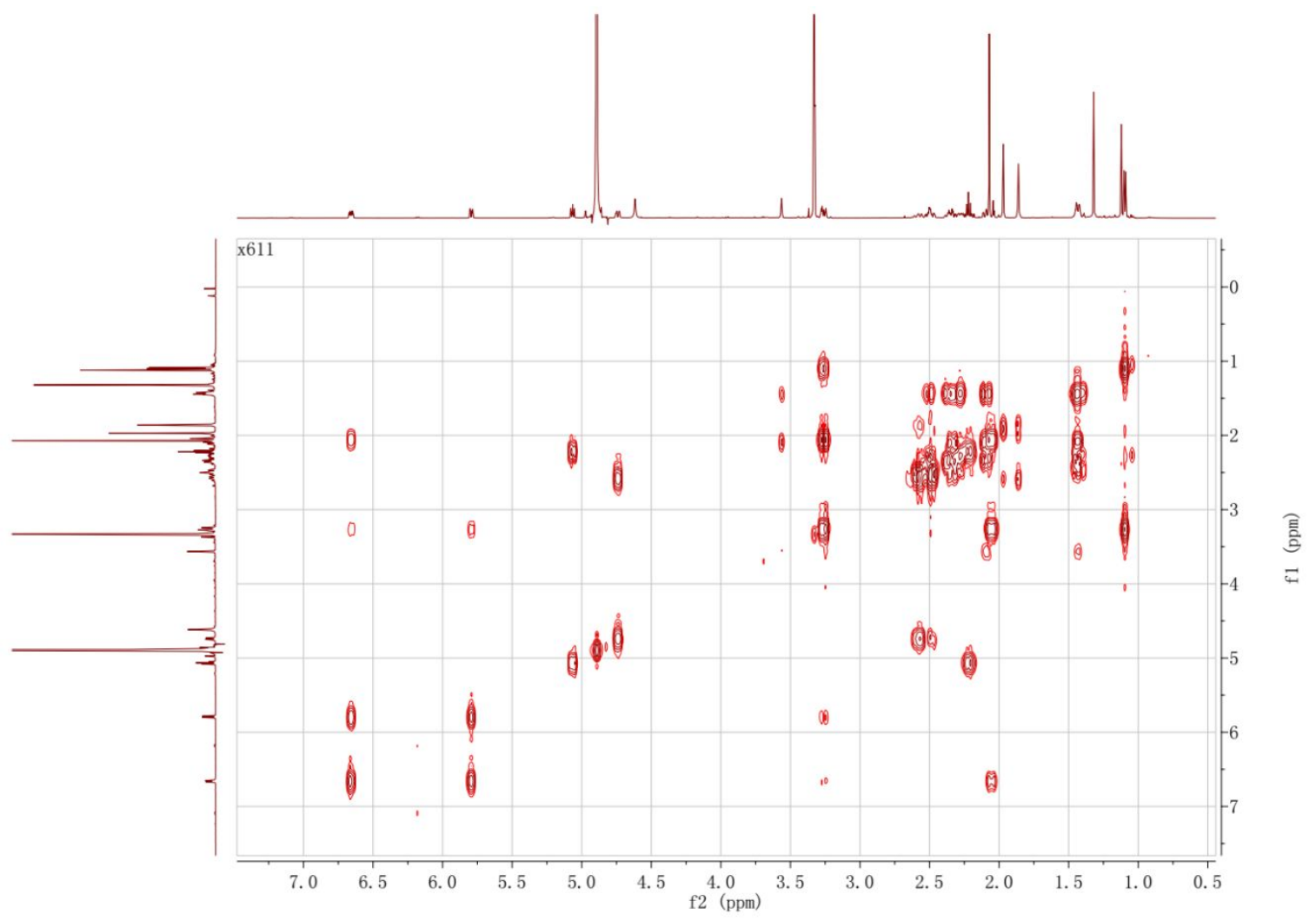




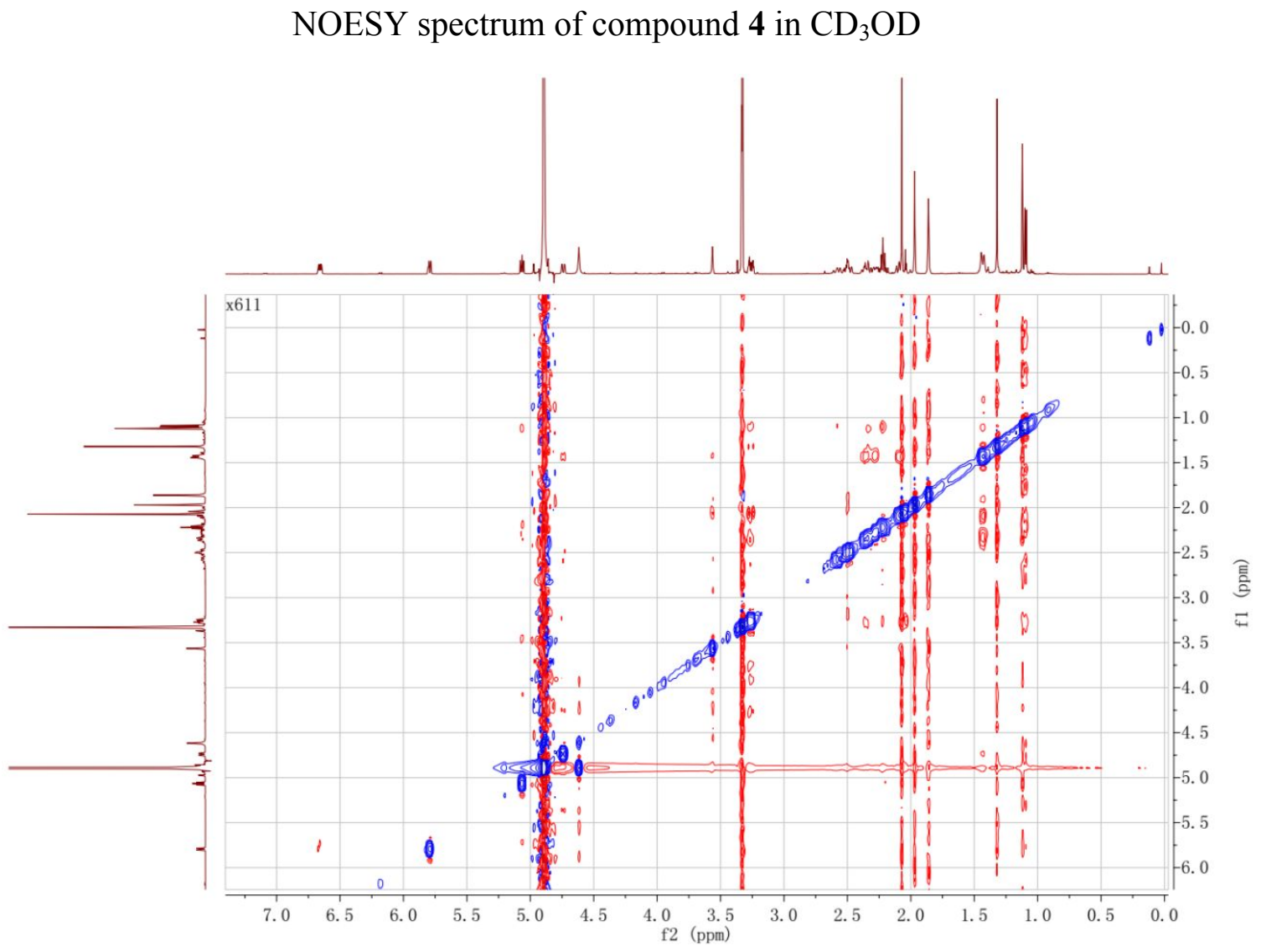


HR-ESI-MS spectrum of compound $\mathbf{5}$

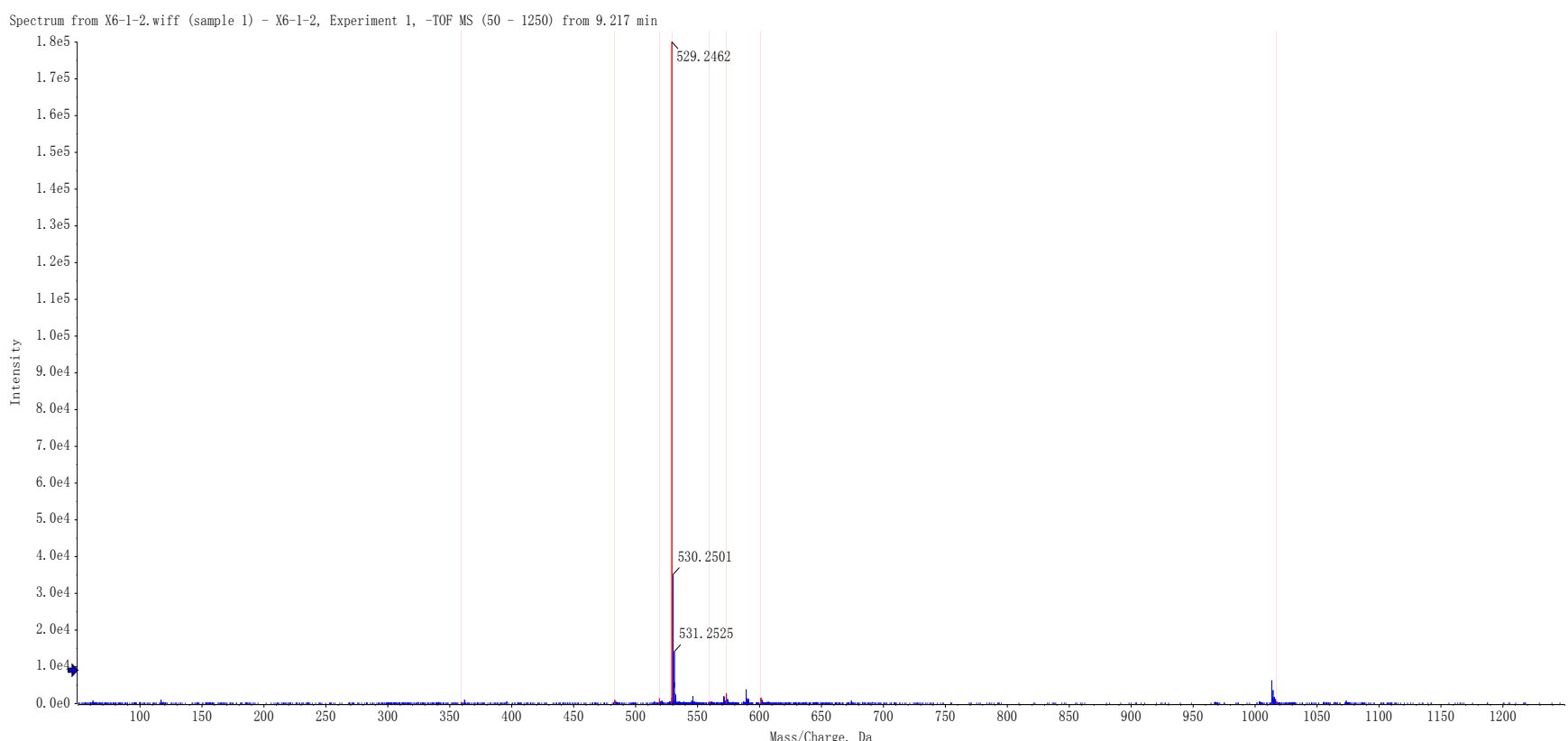

${ }^{1} \mathrm{H}$ NMR spectrum of compound 5 in $\mathrm{CD}_{3} \mathrm{OD}$ at $600 \mathrm{MHz}$

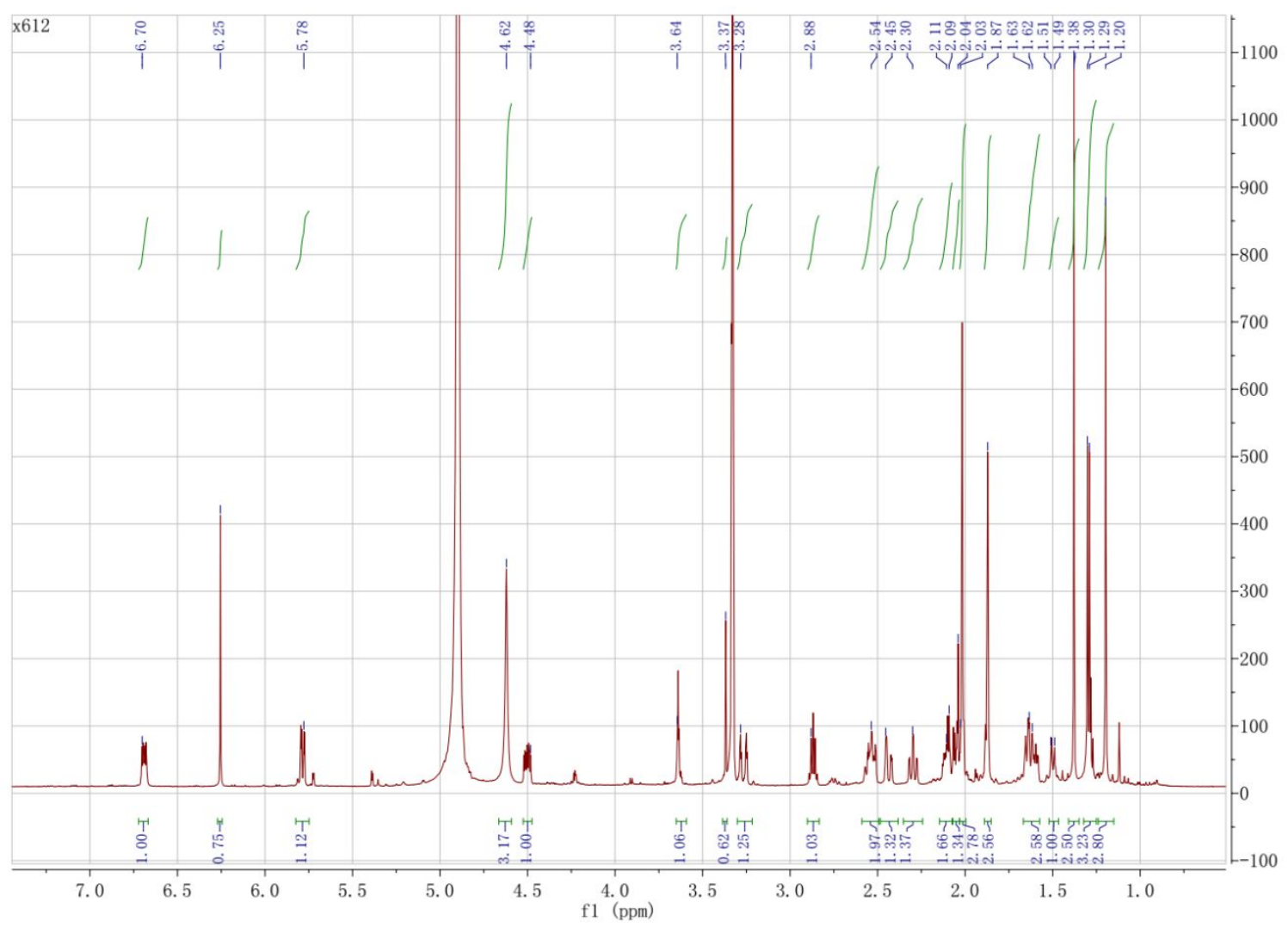


Detailed ${ }^{1} \mathrm{H}$ NMR spectrum of compound 5 in $\mathrm{CD}_{3} \mathrm{OD}$ at $600 \mathrm{MHz}$

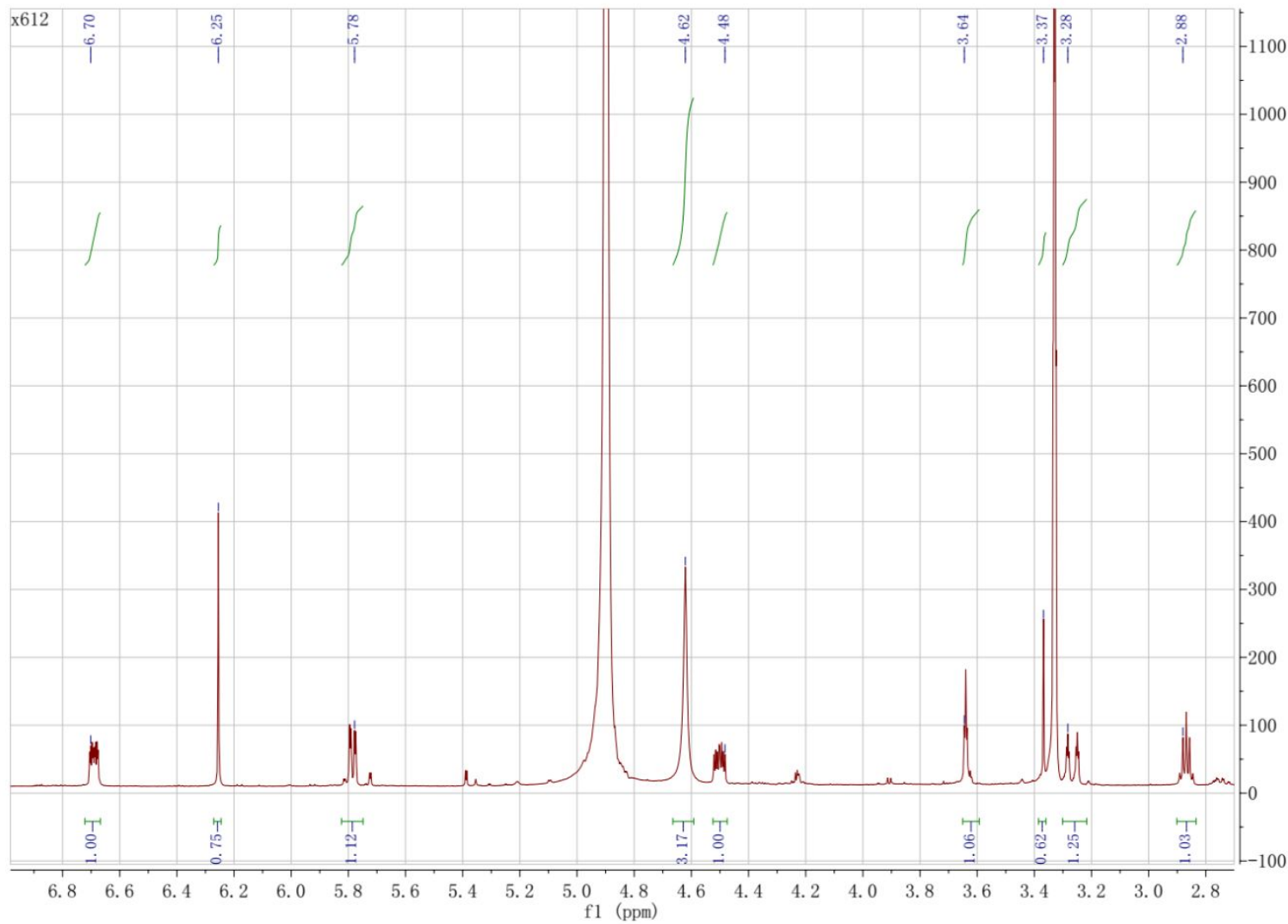

Detailed ${ }^{1} \mathrm{H}$ NMR spectrum of compound 5 in $\mathrm{CD}_{3} \mathrm{OD}$ at $600 \mathrm{MHz}$

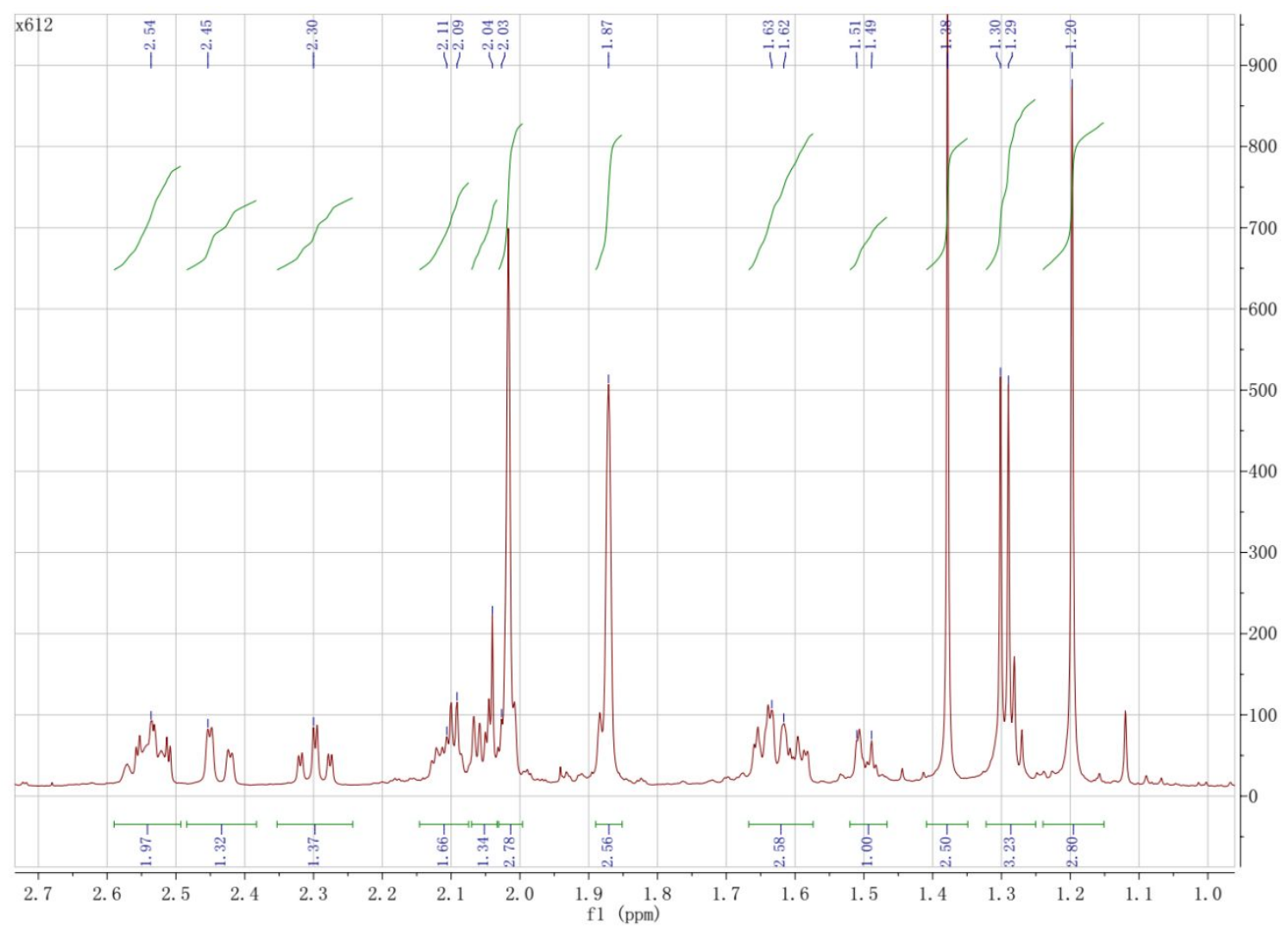


${ }^{13} \mathrm{C}$ NMR spectrum of compound 5 in $\mathrm{CD}_{3} \mathrm{OD}$ at $150 \mathrm{MHz}$

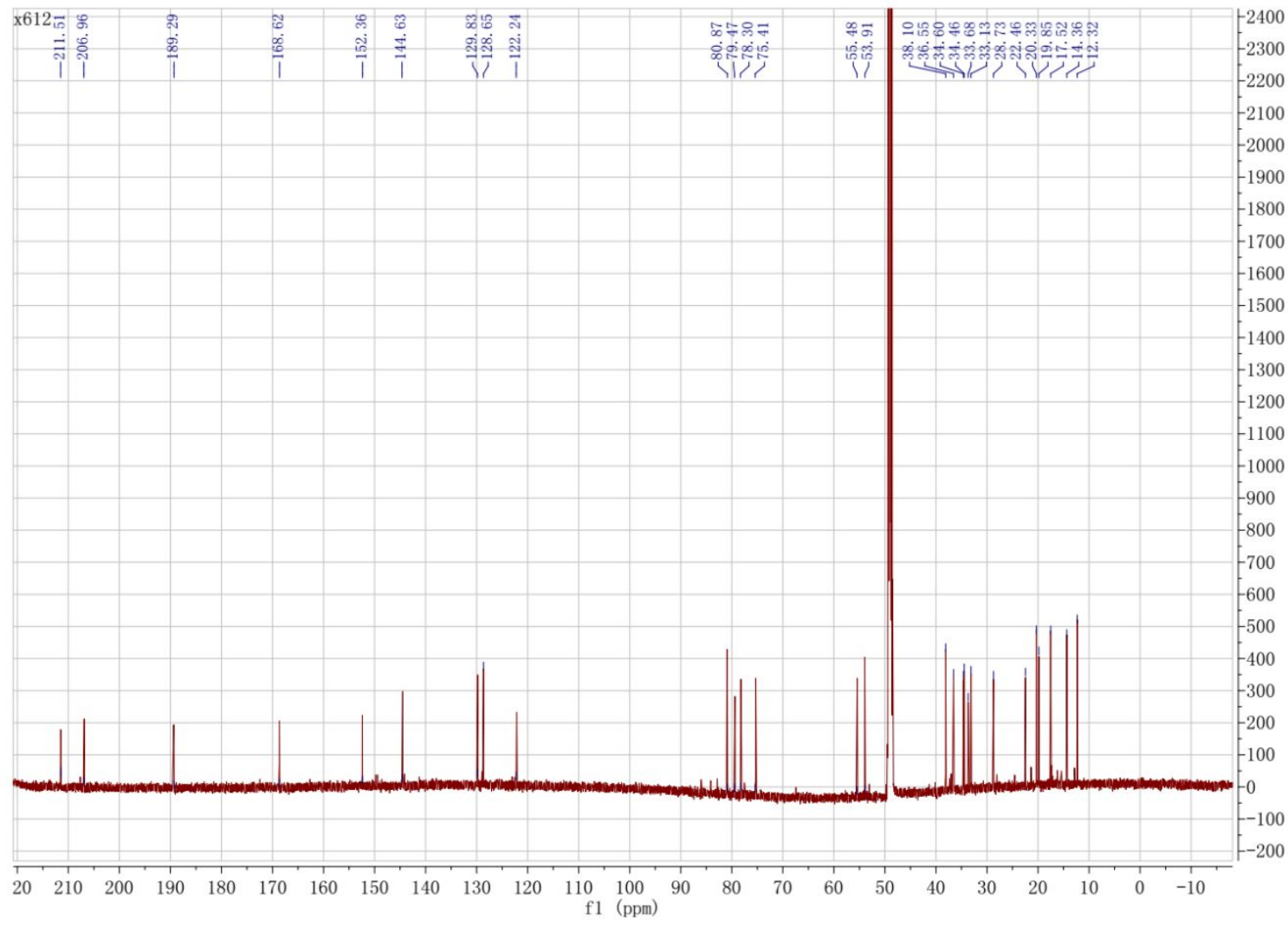

Detailed ${ }^{13} \mathrm{C}$ NMR spectrum of compound 5 in $\mathrm{CD}_{3} \mathrm{OD}$ at $150 \mathrm{MHz}$

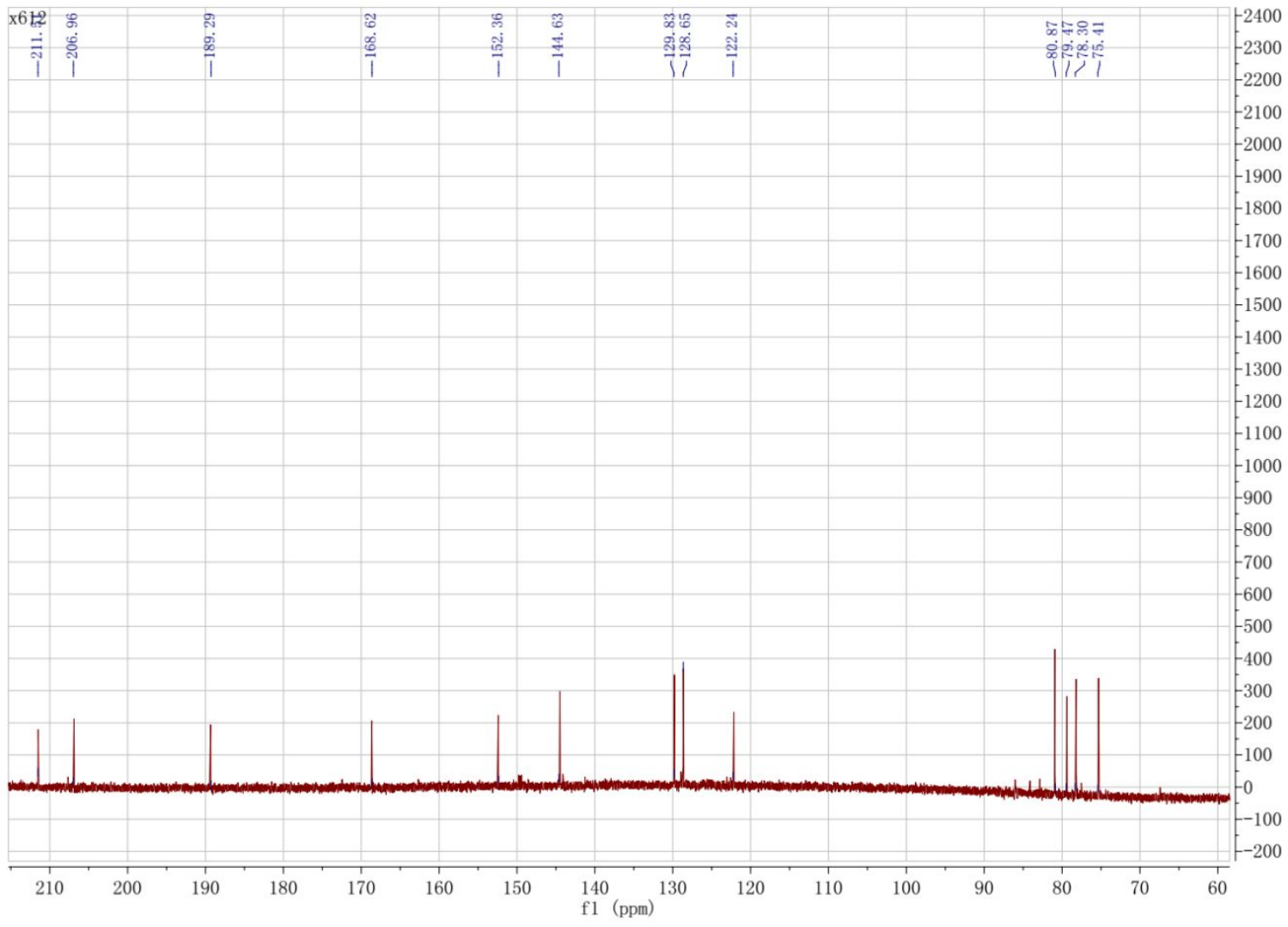


Detailed ${ }^{13} \mathrm{C}$ NMR spectrum of compound 5 in $\mathrm{CD}_{3} \mathrm{OD}$ at $150 \mathrm{MHz}$

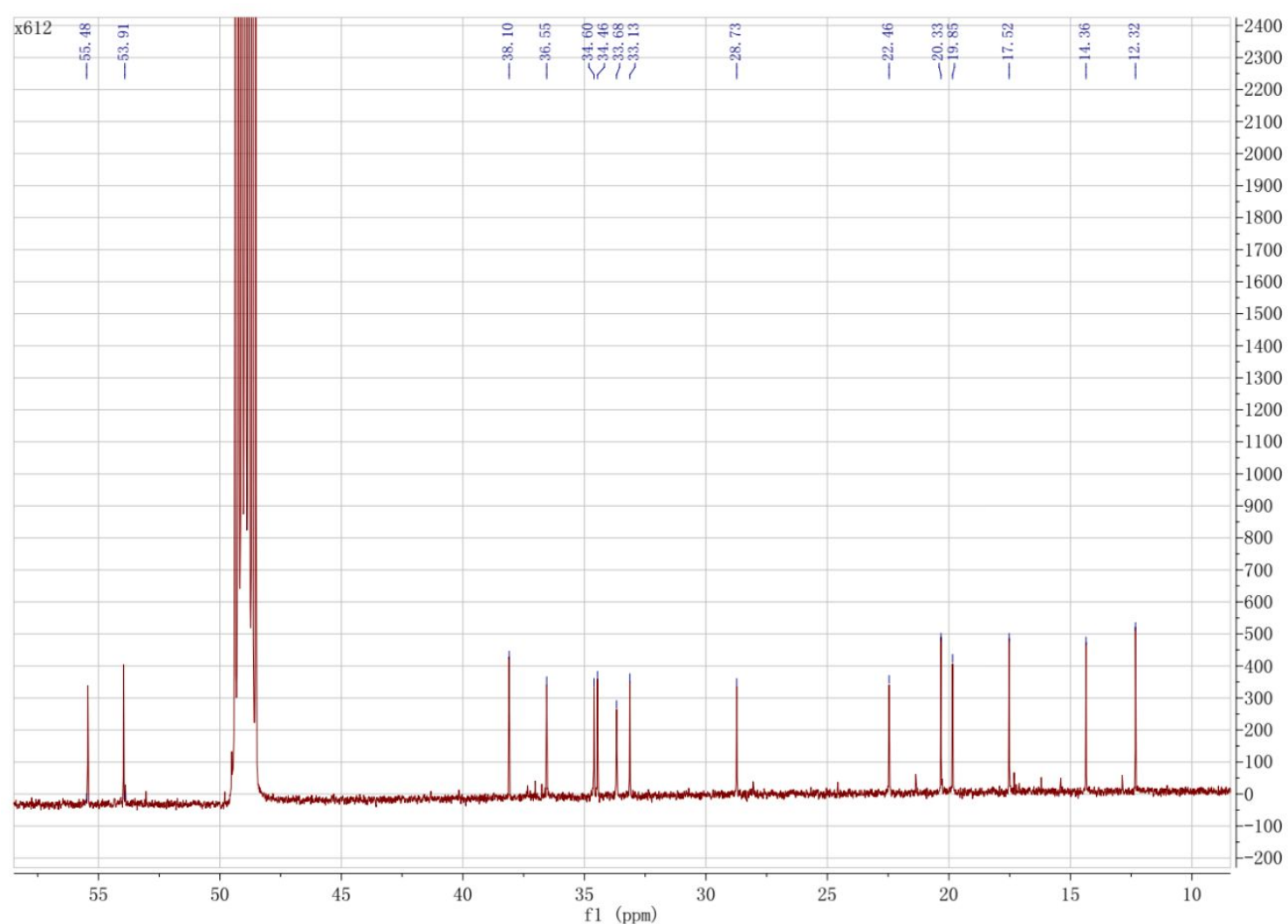

HSQC spectrum of compound 5 in $\mathrm{CD}_{3} \mathrm{OD}$

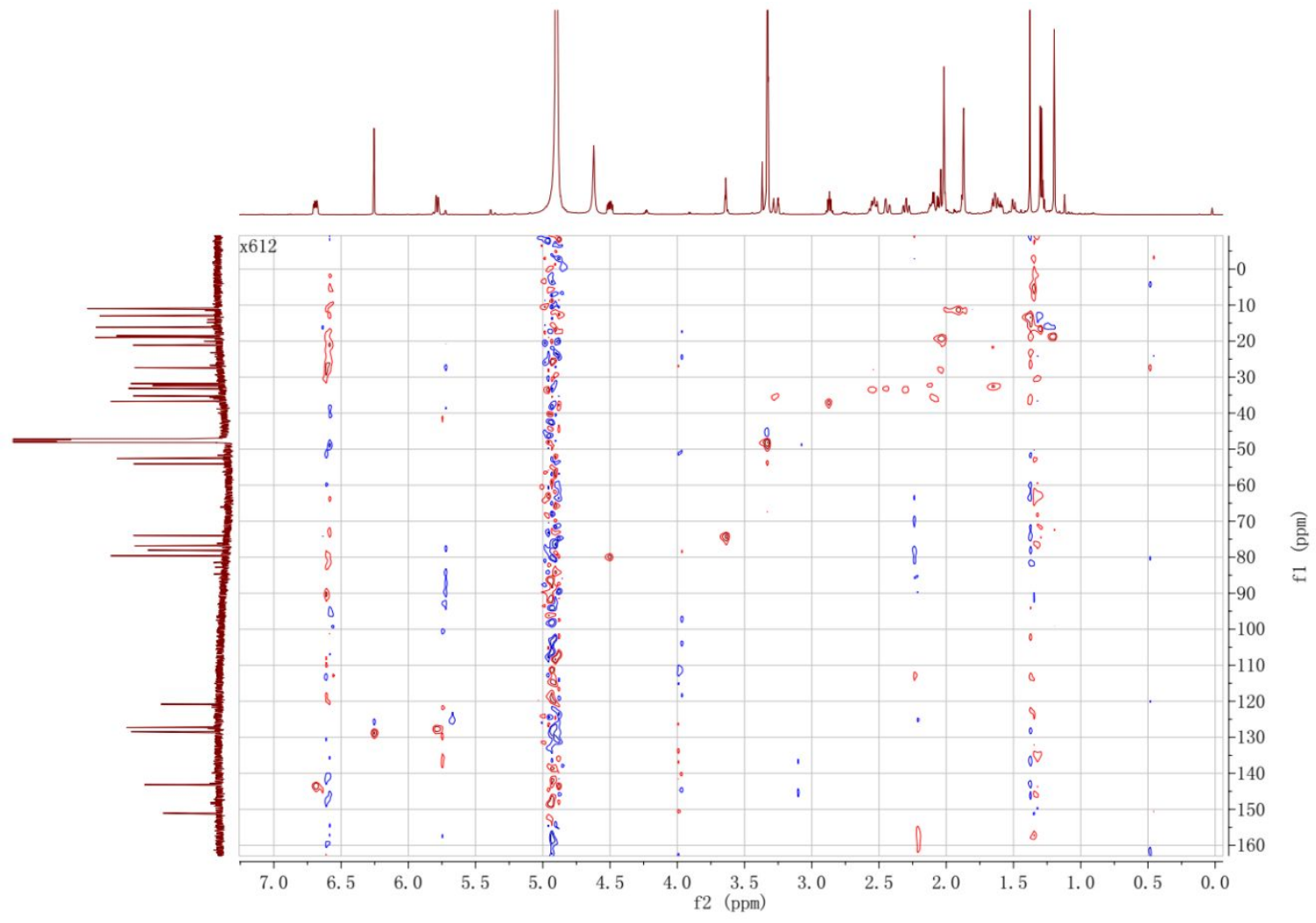


$\mathrm{HMBC}$ spectrum of compound 5 in $\mathrm{CD}_{3} \mathrm{OD}$

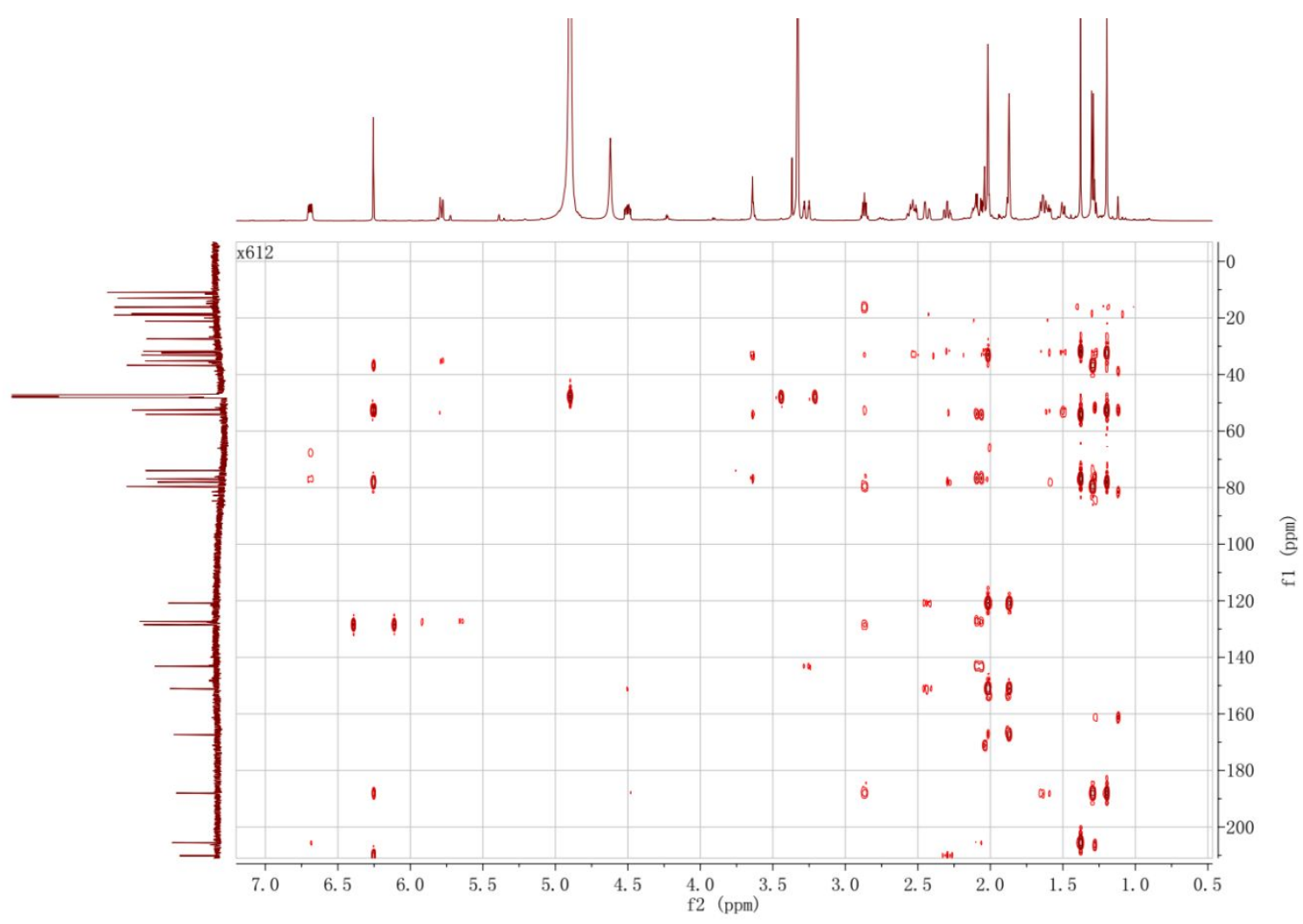

COSY spectrum of compound 5 in $\mathrm{CD}_{3} \mathrm{OD}$

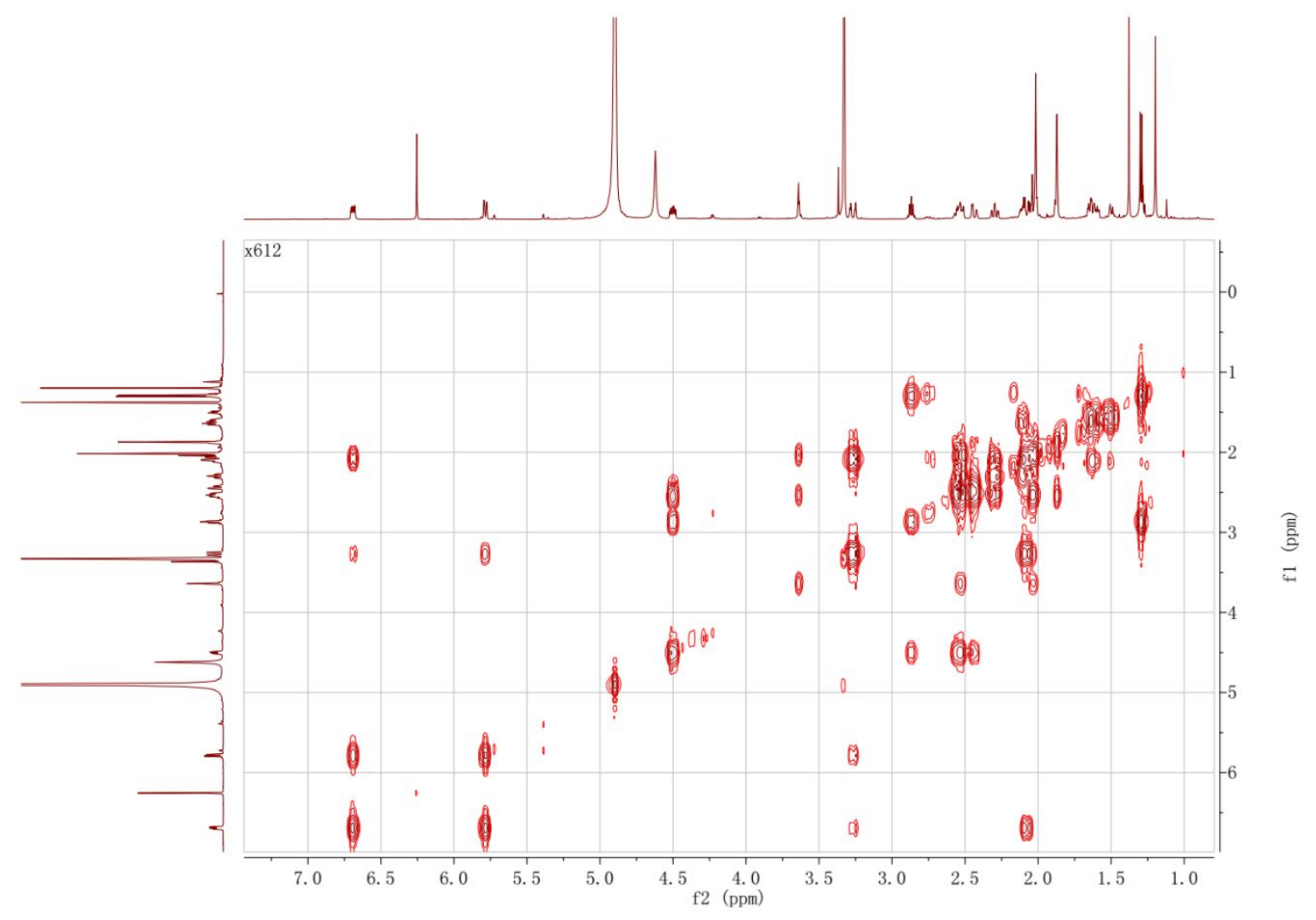


NOESY spectrum of compound 5 in $\mathrm{CD}_{3} \mathrm{OD}$

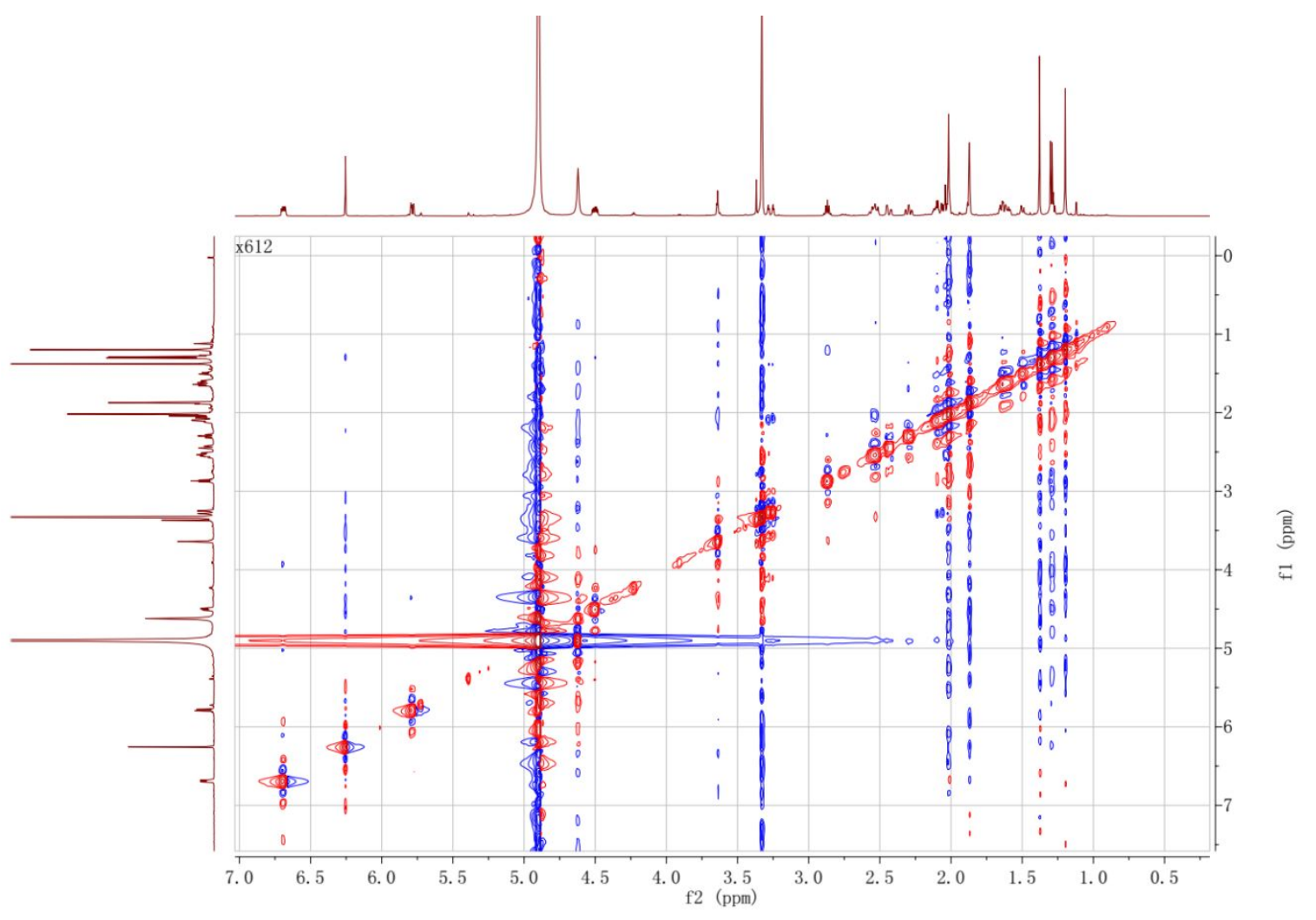

